\title{
The Vestibular Implant
}

Citation for published version (APA):

Guinand, N. (2018). The Vestibular Implant:

a more stable horizon for patients with a bilateral vestibular deficit? [Doctoral Thesis, Maastricht University]. Datawyse / Universitaire Pers Maastricht. https://doi.org/10.26481/dis.20180920ng

\section{Document status and date:}

Published: 01/01/2018

DOI:

10.26481/dis.20180920ng

Document Version:

Publisher's PDF, also known as Version of record

\section{Please check the document version of this publication:}

- A submitted manuscript is the version of the article upon submission and before peer-review. There can be important differences between the submitted version and the official published version of record.

People interested in the research are advised to contact the author for the final version of the publication, or visit the DOI to the publisher's website.

- The final author version and the galley proof are versions of the publication after peer review.

- The final published version features the final layout of the paper including the volume, issue and page numbers.

Link to publication

\footnotetext{
General rights rights.

- You may freely distribute the URL identifying the publication in the public portal. please follow below link for the End User Agreement:

www.umlib.nl/taverne-license

Take down policy

If you believe that this document breaches copyright please contact us at:

repository@maastrichtuniversity.nl

providing details and we will investigate your claim.
}

Copyright and moral rights for the publications made accessible in the public portal are retained by the authors and/or other copyright owners and it is a condition of accessing publications that users recognise and abide by the legal requirements associated with these

- Users may download and print one copy of any publication from the public portal for the purpose of private study or research.

- You may not further distribute the material or use it for any profit-making activity or commercial gain

If the publication is distributed under the terms of Article $25 \mathrm{fa}$ of the Dutch Copyright Act, indicated by the "Taverne" license above, 
(C) Nils Guinand, Maastricht 2018

Layout: Tiny Wouters

Cover design: Jean Scheijen | vierdrie.nl

Production: Datawyse / Universitaire Pers Maastricht

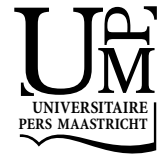

ISBN: $\quad 9789493019768$ 


\title{
The Vestibular Implant: \\ a more stable horizon for patients with a bilateral vestibular deficit?
}

\begin{abstract}
PROEFSCHRIFT
ter verkrijging van de graad van doctor aan de Universiteit Maastricht, op gezag van de Rector Magnificus, Prof. dr. Rianne M. Letschert, volgens het besluit van het College van Decanen,

in het openbaar te verdedigen

op 20 September 2018 om 12.00 uur
\end{abstract}

door

Nils Guinand

geboren op 19 juni 1977 te Bern, Zwitzerland 


\section{Promotores}

Prof. dr. H Kingma, Division of Balance Disorders, Department of Otorhinolaryngology and Head and Neck Surgery, Maastricht University Medical Center, School for Mental Health and Neuroscience, Maastricht, Netherlands and Faculty of Physics, Tomsk State Research University, Tomsk, Russia.

Prof. dr. J-P Guyot, Service of Otorhinolaryngology Head and Neck Surgery, Department of Clinical Neurosciences, Geneva University Hospitals, Geneva, Switzerland.

\section{Beoordelingscommissie}

Prof. dr. B Kremer (voorzitter), Department of Otorhinolaryngology, Head and Neck Surgery, Maastricht University Medical Centre, Maastricht, The Netherlands.

Prof. dr. AAL Jacobi-Postma, Department of Radiology, Maastricht University Medical Centre, Maastricht, The Netherlands

Prof. dr. M Magnusson, Department of Otorhinolaryngology, Head and Neck Surgery,Skåne University Hospital and Lund University, Sweden

Prof. dr. M Strupp, Department of Neurology and German Center for Vertigo and Balance Disorders (DSGZ), Ludwig Maximilian University, Munich, Germany 


\section{Contents}

Chapter 1 Introduction: The vestibular implant, a probe in orbit 7 around the human balance system.

Chapter 2 Quality of life of patients with bilateral vestibulopathy.

Chapter 3 Visual acuity while walking and oscillopsia severity in healthy subjects and patients with unilateral and bilateral vestibular function loss.

Chapter 4 Vestibular implants: 8 Years of experience with electrical stimulation of the vestibular nerve in 11 patients with bilateral vestibular loss

Chapter 5 Restoring visual acuity in dynamic conditions with a vestibular implant

Chapter 6 Final discussion and valorisation

Summary

List of publications

Curriculum vitae

Acknowledgements 



\section{Chapter 1}

The vestibular implant: a probe in orbit around the human balance system

Angelica Perez Fornos, Samuel Cavuscens, Maurizio Ranieri, Raymond van de Berg, Robert Stokroos, Herman Kingma, Jean-Philippe Guyot, Nils Guinand

J Vestib Res 2017;27(1):51-61 


\section{Abstract}

The primary goal of the vestibular implant is to restore the vestibular function in patients with a disabling bilateral vestibular loss for whom there is currently no available treatment. The prototype developed by our team is a hybrid system consisting of a modified cochlear implant incorporating additional vestibular electrodes. Therefore, in addition of delivering sound information it is also capable of delivering motion information to the central nervous system using electrical stimulation. To date, thirteen patients have been implanted with such vestibular implant prototypes. For ethical reasons, only deaf ears were implanted and all patients experienced a clinical benefit from the hearing rehabilitation. The recent demonstration of partial restoration of the vestibulo-ocular and the vestibulo-collic reflexes in implanted patients suggests that gaze stabilization and postural control, fundamental functions of the balance system, can be artificially restored using a vestibular implant. This allows us to glimpse a useful clinical application in a near future. In parallel, we show how the vestibular implant provides a unique opportunity to explore the integration of the vestibular sensory input into the multisensory, multimodal balance system in humans, since it is able to selectively stimulate the vestibular system. 


\section{Introduction}

In general, the society recognizes that the loss of sensory function is an important handicap. However, certain conditions are better recognized and understood than others. Good examples of well recognized, disabling conditions are blindness (loss of sight) and deafness (loss of hearing). In these cases, important efforts in the management of affected patients have been undertaken. Unfortunately, the picture is completely different for other conditions. The handicap suffered by patients with a complete loss of the vestibular function is significantly underestimated and the pathway to diagnosis is full of hurdles. ${ }^{1,2}$ However, recent studies have unambiguously revealed that the complete loss of the vestibular function has a dramatic impact on the quality of life of affected patients. ${ }^{3}$ It also represents significant economic burden for patients and society. ${ }^{4}$

The clinical management of patients with bilateral vestibular loss remains difficult as there is no clear evidence for an effective treatment. Physical therapy showed $n 0^{5}$ or limited benefit. ${ }^{6}$ Other treatment options such as sensory substitution with auditory or vibrotactile feedback have shown contrasting results and are still under clinical investigation. ${ }^{7-9}$ Recent spectacular results showing the successful differentiation of stem cells into functional hair-cell-like cells and auditory neurons that are similar with the vestibular neurosensory cells, allow the wildest hope for the future. ${ }^{10}$ However, it might still be long before this promising therapeutic pathway can be successfully translated to the clinic.

These important facts support the vestibular implant research efforts that have been launched more than 15 years ago primarily to rehabilitate patients with a bilateral vestibular loss. Promising results have been obtained in animal models and humans and partial restoration of the vestibular function has been demonstrated. ${ }^{11-13}$ Moreover, awareness among the medical community, funding agencies, and specialized industry (in particular the cochlear implant companies) has substantially increased in the last years. Hopefully, this rising interest will boost research in the field allowing, in the near future, the availability of a vestibular implant able to restore useful vestibular function and help "normalize" balance in patients with a severe bilateral vestibular loss. Another interesting aspect of vestibular implant research is that this device constitutes a unique opportunity to develop fundamental knowledge in the vestibular research field, in particular by providing unprecedented, selective, and direct access to the vestibular endorgans.

\section{Vestibular function}

The peripheral vestibular organ is located in the inner ear and is composed of five special sensory organs (i.e., endorgans) dedicated to sensing head movements. The three pairs of semicircular canals are sensitive to angular acceleration, providing cues 
about three-dimensional head rotations. The otolithic organs (saccule and utricule) are predominantly sensitive to linear acceleration, providing cues about 3D head translations, including gravity. This motion information allows unambiguous real time monitoring of head position and movement. Unlike other sensory organs, vestibular input quickly becomes multimodal (i.e., multisensory) in subcortical structures, at the level of the second neurons. A direct consequence of this is that there is no clear conscious or distinct sensation coming from the vestibular endorgans alone. Yet, vestibular information is involved in a large variety of subcortical and cortical functions, which is illustrated by the multifocal brain activation observed to different vestibular stimuli. ${ }^{14}$

Vestibular information is fundamental for a variety of functional aspects. Vestibular reflexes play a central role in the balance system. The vestibulo-ocular reflex (VOR) mediates gaze stabilization in dynamic conditions, while the vestibulo-spinal (VCS) and vestibulo-collic reflexes (VCC) are important for postural control. Motion perception is another function that relies on complex integration of vestibular input. For example, integration of canal and otolithic information in an internal model is necessary to solve the ambiguity between a tilt and a translation in the horizontal plane. ${ }^{15} \mathrm{We}$ also know that motion related information affects the activity of hippocampal place cells which are essential for spatial navigation. ${ }^{16,17}$ Moreover, the vestibular input interacts with the autonomic system for the regulation of arterial blood pressure during gravity changes, ${ }^{18}$ or during head-up tilt. ${ }^{19}$ There is even some evidence that vestibular dysfunction could play a role in orthostatic hypotension. ${ }^{20}$ Other authors have shown interactions with the respiratory system ${ }^{21}$ and with sleep patterns. ${ }^{22}$ Finally, the multiple vestibular projections are known to influence emotions, memory, cognition $^{23,24}$ and even personality. ${ }^{25}$

\section{Bilateral loss of the vestibular function}

In patients with a bilateral vestibular loss, imbalance is probably the predominating symptom. However, the global clinical picture involves functional impairments at different levels inducing a variety of symptoms which are not necessary flagrant. Additionally, as previously mentioned, there is no clear conscious sensation of the vestibular endorgans. This makes it often difficult for patients to understand and describe their symptoms and, consequently, clinicians face confusing anamnesis.

\section{Vestibular testing}

Despite significant progress in vestibular testing, it remains a clinical challenge to assess and quantify the vestibular function in its entirety. We still mostly rely on the evaluation of vestibular reflexes (i.e., VOR measurement to a variety of stimuli for canal function and vestibular evoked myogenic potentials recording for otholithic 
function). However, various other diagnostic approaches are being explored. Assessment of multisensory tasks such as visual acuity in dynamic conditions (i.e., while walking or during unpredictable passive head movements) is a sensitive test of the integration of vestibular input into the balance system. ${ }^{26-28}$ The measurement of motion perception thresholds is another strategy to assess the functionality of the vestibular system with a long history in vestibular research. Significant progress and interesting results have been achieved with these tests, ${ }^{29}$ and some experts in the field envision them to soon become part of the standard vestibular testing battery. However significant remaining drawbacks of the method are the necessary heavy and expensive equipment (i.e., hexapod), the duration of the test (many hours) and the fact that it is not possible to completely rule out the effect of somatosensory cues. Posturography has also been extensively studied and is used by many clinicians. However, a group of experts in the field recently concluded that there is no generally applicable posturography test providing reasonable sensitivity and specificity for the diagnosis of balance disorders currently available. ${ }^{30}$ They also concluded that perturbation techniques are most likely needed to enhance the diagnostic yield of this technique.

In summary, significant challenges still need to be overcome in the field of vestibular testing and evaluation. In addition, the quantification of the "artificial" vestibular information provided by a vestibular implant is a recent endeavor. This is why one of the main challenges in the vestibular implant field will be the development of meaningful tests to objectively determine its clinical benefit. In this paper we attempt to summarize the milestones of our group in this direction.

\section{Methods}

\section{The concept of the vestibular implant}

The concept is similar to that of the cochlear implant except that instead of sound, motion information is captured using head-fixed sensors and fed to a special processor $^{31}$ where it is converted into an appropriate neural pattern. This pattern is is in then transmitted to the implanted stimulator via telemetry. The stimulator consists of a modified cochlear implant with 1 to 3 "vestibular" electrodes taken out of the cochlear electrode array and put into separate branches designed to be positioned closely to the ampullary branches of the vestibular nerve (Figure 1.1). Finally, the neural patterns of processed motion information are provided to the central nervous system in the form of electrical currents delivered via the vestibular electrodes. 


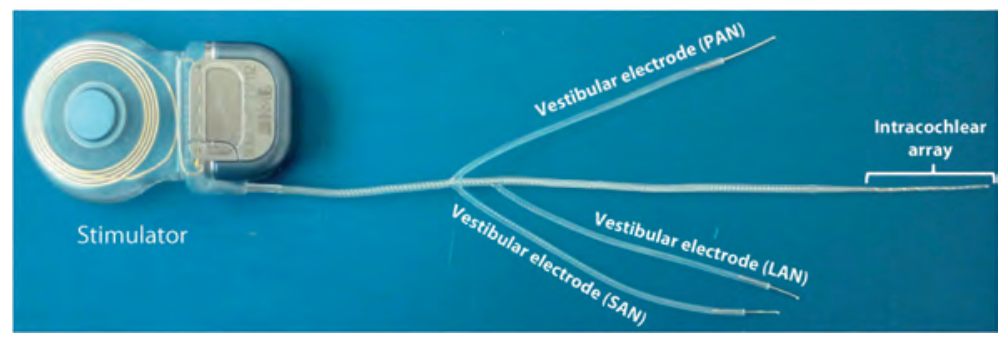

Figure 1.1 A multichannel version of the vestibular implant prototype, consisting in a modified cochlear implant (Medel, Innsbruck, Austria) with 3 vestibular electrodes to be implanted in the 3 semicircular canals close to the corresponding ampullary nerves and the main array with cochlear electrodes.

In this concept, the vestibular implant aims at transmitting "artificial" neural patterns to the central nervous system similar to those coded by the normally functioning vestibular system. In a healthy subject, the vestibular nerves fire spontaneously at a rate of about 90 action potentials/s in the absence of movement. ${ }^{32}$ Motion is then coded by the modulation of this spontaneous firing rate. For example, for the horizontal semicircular canal a head rotation in the direction of the canal (i.e., rightwards for the right ear and leftwards for the left ear) will result in an increase of the firing rate. Conversely, a horizontal head rotation in a direction opposite to the canal (i.e., leftwards for the right ear and rightwards for the left ear) will result in a decrease of the firing rate. This motion-controlled modulation of the spontaneous firing rate results in a compensatory horizontal eye movement in the direction opposite to the head movement. Therefore, in patients with a bilateral loss of the vestibular function, a prerequisite to reestablish bi-directional eye movements with unilateral electrical stimulation is to restore an artificial "spontaneous" firing rate so that it can be increased (up-modulated) for generating eye movements in one direction and decreased (down-modulated) for generating eye movements in the opposite direction.

\section{Development of surgical techniques}

A fundamental step was the development of appropriate surgical techniques to allow for stable and safe implantation of electrodes in the vicinity of the vestibular nerve branches. However, this surgical access presents significant risk of inducing hearing loss. $^{33}$ As the majority of BVL patients has a normal or residual hearing, hearing preservation represents a major issue. Therefore, an extralabyrinthine surgical approach was initially developed based on the known surgical approach to the posterior ampullary nerve developed by Gacek who showed results with a high rate of 
hearing preservation (>95\%). ${ }^{34}$ A first series of 100 temporal bones were dissected, showing a procedure success rate of $98 \%$ in accessing the posterior ampullary nerve. ${ }^{35,36}$ The need to elicit eye movements in other "orthogonal" directions led to the development of similar extralabyrinthine surgical approaches to the superior and lateral ampullary nerves. In a second series of 80 temporal bones, both structures could be reached in 71 temporal bones. Nevertheless, in most of the cases (64/71) the ampulla was opened. ${ }^{37}$ All the above mentioned approaches are transmeatal (through the external auditory canal) and can be done in local anesthesia which allowed acute intra-operative testing in the awake patient which was essential to demonstrate the feasibility of the project.

As already mentioned, the extralabyrinthine approaches were thought to be safer and were initially favored. Nevertheless, all the promising results reported by research groups working in the same field on animals were obtained by using more classical intralabyrinthine approaches. ${ }^{12,38}$ Therefore, our group also developed an intralabyrinthine surgical approach. ${ }^{39}$ A mastoidectomy is performed, each semicircular canal is exposed and a micrometric labyrinthotomy close to the ampulla is performed. While a certain degree of hearing and vestibular function preservation was obtained in animal experiments after intralabyrinthine electrode insertion, this could not be confirmed in human experiments. ${ }^{40-44}$ Therefore, the risk of impairing hearing by using an intralabyrinthine approach remains unknown. Nevertheless, promisingly, it has been shown that it is possible to preserve hearing during canal plugging, which is a similar surgical procedure performed for intractable benign paroxysmal positional vertigo or for severe Menière's disease. ${ }^{45-47}$

\section{Ethical considerations}

All experiments were designed and conducted in accordance with the 1964 Declaration of Helsinki. Local ethical committees of the Geneva University Hospitals (NAC 11-080) and of the Maastricht University Medical Centre (NL36777.068.11/METC 11-2-031) approved this experimental protocol. All participants gave their informed consent prior their inclusion in the study.

\section{Results}

\section{Acute intra-operative stimulation trials}

After having developed the surgical routes to reach the ampullary branches of the vestibular nerve, the first step was to show that vestibular responses could be elicited in human patients in acute stimulation trials during surgeries where the vestibular nerves were accessible. To do this, 3 deaf patients, candidates to a cochlear 
implantation, were selected. Two patients had a bilateral Menière's disease and one patient had a concomitant unilateral vestibular loss. In all 3 cases, the posterior ampullary nerve was accessed (extralabyrinthine approach) in local anesthesia. An electrode was positioned in its contact and maintained manually in place to allow for acute electrical stimulation. Robust nystagmic responses reflecting the activation of the vestibular system were elicited and were recorded using a standard 2D videooculography system. Those nystagmic responses showed a threshold effect and were predominantly in the vertical plane. For a given pulse rate, a monotonic, fairly linear correlation between current amplitude $(\mu \mathrm{A})$ and slow phase velocity of the nystagmus was found. In a second stage, current amplitude was kept constant and its pulse rate was progressively increased. The observed nystagmic responses also showed a threshold effect. The amplitude of the nystagmus slow phase velocity showed an almost linear rise with a peak at an "optimal" pulse rate followed by a progressive decline.

During the acute stimulation trials, the patients described a feeling of surprise, then a sensation of rotatory motion without nausea or motion sickness. At the end of the experimental procedure, general anesthesia was induced and a regular cochlear implantation was performed. ${ }^{48}$

A similar procedure targeting the lateral and superior ampullary branches of the vestibular nerve (extralabyrinthine) was done in three patients with unilateral intractable Menière's disease prior to a transmeatal labyrinthectomy. Hearing was non serviceable in the operated ears. The recorded nystagmic responses were predominantly in the horizontal plane without concomitant stimulation of the facial nerve. $^{49}$

Using the intralabyrinthine surgical approach described by van de Berg et al., ${ }^{39}$ the first acute intra-operative intralabyrinthine electrical stimulation trials of the different ampullary branches of the vestibular nerve were performed in 3 deaf patients with a bilateral vestibular loss prior to cochlear implantation. ${ }^{39}$ Note that the procedure was performed under general anesthesia which is known to impair nystagmic responses, especially the fast phase of the VOR. ${ }^{50,51}$ Particularly propofol (2,6-diisopropylphénol), a short acting hypnotic agent used to induce and maintain general anesthesia, has been shown to decrease significantly the vestibular nuclei activity in rats. ${ }^{52}$ Therefore, prior to electric stimulation trials, propofol was stopped and anesthesia was maintained with remiphentanil, a short-acting synthetic opioid, similar to fentanyl. As fentanyl selectively suppresses the fast phase of the nystagmic response, ${ }^{53}$ only the slow phases of the VOR could be electrically elicited, resulting in a tonic deviation of the eyes. The observed tonic deviations were predominantly in the plane of the stimulated semicircular canal. As the electric stimulation was stopped, the eyes returned to the neutral position, presumably driven by elastic restoring forces of the eyeball. No complications were reported in the follow up. 


\section{Chronic implantations and adaptation to electrical stimulation of the vestibular system in humans}

The first implantation of a vestibular implant prototype in a human was performed in Geneva in 2007. The patient suffered from bilateral deafness and concomitant idiopathic bilateral vestibular loss. This first vestibular implant prototype incorporated only one vestibular electrode together with the cochlear array. The posterior ampullary nerve could not be visually identified during surgery, but the vestibular electrode was positioned in its vicinity according to the known landmarks. As the surgery was done in general anesthesia, no attempt of generating vestibular responses upon intra-operative stimulation trials was made. Postoperative electrical stimulation delivered via the vestibular electrode elicited robust nystagmic responses with a dominant horizontal component, indicating a possible current spread to other branches of the vestibular nerve. During stimulation, the patient reported a high frequency sound and a sensation of dizziness, both of which increased with increasing current intensity.

Since the concept of the vestibular implant is to mimic the physiology of the vestibular system, the next step consisted of attempting to restore an "artificial" baseline or "rest" activity in the vestibular nerve. After approximately 27 minutes of constant electrical stimulation the nystagmic responses induced at the sudden onset of stimulation almost completely vanished. This was the first demonstration of adaptation to constant electrical stimulation of the vestibular nerve in a human subject. Moreover a reversed nystagmic response, lasting less than 10 minutes, was observed when the stimulation was suddenly turned off. After several repetitions of such on/ off stimulation sequences, the adaptation time required for nystagmic responses to disappear fell down to less than 5 minutes. This "adaptation" lasted close to 24 hours after the device has been turned off ${ }^{54}$ and was much faster than what had been reported in similar animal experiments. ${ }^{55}$ These findings had crucial implications for the clinical application of the concept, significantly reducing concerns that a vestibular implant had to be permanently activated to avoid long-lasting adverse symptoms when turning the device on or off. Finally, another fundamental finding was the demonstration of the ability to generate smooth, controlled eye movements by up- and down- modulating the baseline stimulation using a virtual motion sinusoidal profile. Although both amplitude and frequency modulation were effective, larger responses were recorded with amplitude modulation. ${ }^{56}$

Since this first-of-its-kind experiment performed in 2007, a group of 12 additional patients (i.e., 13 successful implantations in total) have been implanted with 3 incremental generations of vestibular implant prototypes incorporating 1,2 , or 3 vestibular electrodes (see Table 1.1). All patients suffered from severe bilateral vestibular loss and were deaf on the implanted ear (details on the inclusion criteria can be found $\mathrm{in}^{13}$ ). Diverse etiologies and different deficit durations were deliberately 
chosen in order to assess efficacy in a wide patient population. To date, we have no surgical complications or adverse events related to the implantations to report. All details regarding implantations and results obtained with the 11 first implanted patients have been recently reported. ${ }^{57}$

Table 1.1 Demographic details and implantation characteristics of the implanted patients.

\begin{tabular}{|c|c|c|c|c|c|c|c|c|}
\hline $\begin{array}{l}\text { Patient } \\
(\mathrm{N}=13) \\
\end{array}$ & Sex & Etiology & Onset & $\begin{array}{l}\text { Age } \\
\text { (implant) }\end{array}$ & $\begin{array}{l}\text { Year } \\
\text { implanted }\end{array}$ & Side & $\begin{array}{l}\text { Vestibular } \\
\text { electrodes }\end{array}$ & $\begin{array}{l}\text { Surgical } \\
\text { approach }^{\bullet}\end{array}$ \\
\hline BVL1 & $\mathrm{m}$ & Idopathic & Progressive & 68 & 2007 & left & PAN & $\mathrm{EL}$ \\
\hline BVL2 & $\mathrm{m}$ & $\begin{array}{l}\text { Congenital/ } \\
\text { idopathic }\end{array}$ & Progressive & 34 & 2008 & right & PAN & EL \\
\hline BVL3 & $\mathrm{m}$ & $\begin{array}{l}\text { Congenital/ } \\
\text { idopathic }\end{array}$ & Progressive & 46 & 2008 & left & PAN & EL \\
\hline BVL4 & $\mathrm{m}$ & $\begin{array}{l}\text { Sudden hearing } \\
\text { loss right/ } \\
\text { Menière } \\
\text { disease left }\end{array}$ & Progressive & 71 & 2011 & left & PAN & EL \\
\hline BVL5 & $\mathrm{m}$ & Traumatic & $\begin{array}{l}\text { Acute } \\
\text { (<1 year) }\end{array}$ & 63 & 2012 & right & PAN/LAN & EL \\
\hline BVL6 & $f$ & $\begin{array}{l}\text { St.p. } \\
\text { mastoidectomy } \\
\text { in childhood } \\
\text { right/traumatic }\end{array}$ & $\begin{array}{l}\text { Acute } \\
\text { (<1 year) }\end{array}$ & 67 & 2013 & left & $\begin{array}{l}\text { PAN/LAN/ } \\
\text { SAN }\end{array}$ & IL \\
\hline BVL7 & $f$ & $\begin{array}{l}\text { St.p. meningitis } \\
\text { at age of } 1\end{array}$ & $\begin{array}{l}\text { Acute } \\
\text { (47 years) }\end{array}$ & 48 & 2012 & right & $\begin{array}{l}\text { PAN/LAN/ } \\
\text { SAN }\end{array}$ & IL \\
\hline BVL8 & $\mathrm{m}$ & DFNA9 & Progressive & 67 & 2012 & left & $\begin{array}{l}\text { PAN/LAN/ } \\
\text { SAN }\end{array}$ & IL \\
\hline BVL9 & $f$ & DFNA9 & Progressive & 68 & 2013 & left & $\begin{array}{l}\text { PAN/LAN/ } \\
\text { SAN }\end{array}$ & IL \\
\hline BVL10 & $\mathrm{m}$ & DFNA9 & Progressive & 66 & 2013 & left & $\begin{array}{l}\text { PAN/LAN/ } \\
\text { SAN }\end{array}$ & IL \\
\hline BVL11 & $\mathrm{m}$ & DFNA9 & Progressive & 64 & 2013 & left & $\begin{array}{l}\text { PAN/LAN/S } \\
\text { AN }\end{array}$ & IL \\
\hline BVL12 & $\mathrm{m}$ & Traumatic & $\begin{array}{l}\text { Acute } \\
\text { (3 years) }\end{array}$ & 53 & 2015 & right & $\begin{array}{l}\text { PAN/LAN/ } \\
\text { SAN }\end{array}$ & IL \\
\hline BVL13 & $f$ & Idopathic & Progressive & 56 & 2016 & Left & $\begin{array}{l}\text { PAN/LAN/ } \\
\text { SAN }\end{array}$ & IL \\
\hline
\end{tabular}

*PAN- posterior ampullary nerve; LAN - lateral ampullary nerve; SAN- superior ampullary nerve. ${ }^{\circ}$ EL extralabyrinthic; IL - intralabyrinthic

\section{Restoration of the VOR}

The VOR can be considered a very useful window to the functionality of the peripheral vestibular endorgans. Although it only represents a single aspect of the vestibular function, it is the most accessible method to its quantitative assessment. Therefore, most preliminary work on vestibular implants, including ours, initally focused on the VOR to establish feasibility. 
In 3 vestibular implant recipients, the VOR resulting from motion modulated electrical stimulation via the electrode positoned in ampulla of the lateral canal was assessed. ${ }^{13}$ Patients were rotated around an earth vertical axis using a rotatory chair in complete darkness. The rotation profiles of the rotatory chair were of sinusoidal form, with a peak velocity of $30 \%$ and frequencies ranging from $0.1 \mathrm{~Hz}$ up to $2 \mathrm{~Hz}$. The VOR was measured using a 2D video eye-tracker in two conditions: (1) when the device was turned on (system ON) and (2) when the device was not activated (system OFF). With the system $O N$, the VOR gain significantly increased at rotation frequencies of 0.5, 1 and $2 \mathrm{~Hz}$, but not at the lower frequencies of 0.1 and $0.25 \mathrm{~Hz}$. This frequencydependency of the "artificial" VOR was similar to that observed in a group of normal controls. ${ }^{58}$ Increasing the modulation depth (i.e., stimulation intensity) up to $75 \%$ of the available dynamic range further increased the VOR gain. In the best case, the "artificial" VOR was practically normalized (see example in Figure 1.2). Although these experiments were carried out in only a small number of patients and were limited for a small range of motion profiles, these results represented a fundamental milestone, being the first demonstration of functional rehabilitation of the vestibular system with a vestibular implant prototype.
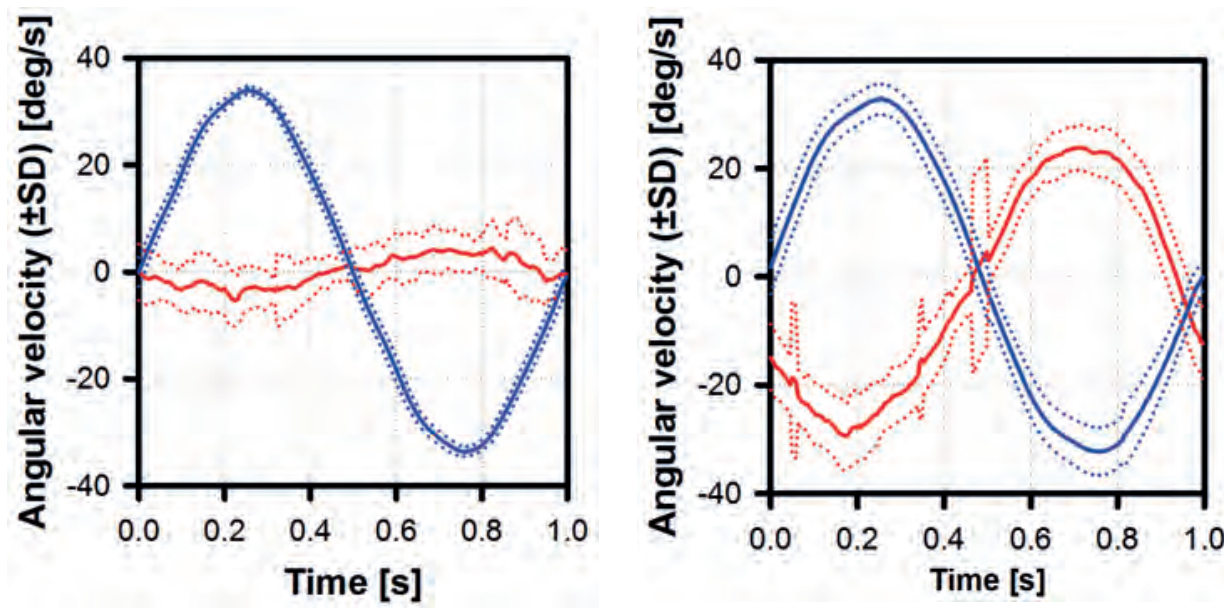

Figure 1.2 Patient with a bilateral vestibular loss, fitted with a vestibular implant prototype, rotating around an earth vertical axis $\left(f=1 \mathrm{~Hz}, \omega \max =30^{\circ} / \mathrm{s}\right)$. A: "system off", B: "system on" (motion modulated stimulation via the electrode positioned close to the lateral ampullary nerve).

The functional implications of the "artificial" restoration of the VOR were additionally measured using visual acuity measurements during electrical stimulation of the posterior or the superior ampullary nerves with a virtual sinusoidal motion profile. In other words, in these experiments the patients were sitting static on a chair while the 
vestibular implant was activated. In this condition, we observed pathological decreases of visual acuity of similar magnitude to dynamic visual acuity drops reported for patients with bilateral vestibular loss. ${ }^{59}$ Deteriorating visual acuity is obviously not the goal of the vestibular implant, but this result is important since it demonstrates that the "artificial" VOR can significantly impact visual acuity. Therefore, if the "artificial" VOR is appropriately fine-tuned, it could provide useful motion information to improve visual performance in simple, everyday dynamic tasks such as during walking. This was further corroborated in pilot measurements of dynamic visual acuity with the vestibular implant prototype, where results clearly showed that the performance of implanted patients became close to normal when coherent motion information was delivered by the device. ${ }^{60}$

\section{Beyond the VOR}

Substantial efforts have been devoted to vestibular implant research in animal models. In particular, innovative psychophysical methods have been developed in order to assess vestibular percepts beyond the VOR (e.g., tilt and head perception perception in primates $\left.{ }^{61-63}\right)$. However, these assessments remain limited in animals, especially because subjective descriptions cannot be obtained from them for obvious reasons. Therefore, research in human patients fitted with a vestibular implant represents a unique opportunity to bring the exploration of the vestibular system to another level.

In a recent paper, the percepts elicited by electrical stimulation of the ampullary branches of the vestibular nerve in 11 patients fitted with a vestibular implant were reported. ${ }^{57}$ Only a few electrodes elicited motion percepts, despite the fact that it would have been the most intuitive guess. In the meantime, two additional patients have been implanted. One suffered a bilateral vestibular loss following trauma with bilateral intraotic temporal bone fractures (BVL12 in Table 1.1). This patient in particular is a clever physicist who understands very well the ongoing experiments and provides very precise descriptions of the effects of electrical stimulation. For example, as the current amplitude of the stimulus delivered to the superior ampullary nerve was gradually increased (25-50 $\mu \mathrm{A}$ increments, stimulation profile identical to previous reports ${ }^{13,57}$ ), he described: "I have never experienced something like this before, I can feel it is there but I cannot explain exactly, I can say it is like a frequency, $20 \mathrm{~Hz}$, but I cannot say this is it. The closest I can get, is in fact, if you suddenly remember something you have forgotten." However, as soon as a cochlear electrode was activated he described: "I can hear, it is a sound!" He was then asked if the sensation he experienced during activation of the "vestibular" electrode had anything to do with sound, he replied without ambiguity: "no it was not a sound!" Given all these unexpected descriptions, it could be questioned whether vestibular nerves had really been activated in our implanted patients. However, the placement of electrodes 
under visual control using known surgical approaches, the radiologic control of the electrode position, and the presence of a VOR during electrode activation are all strong arguments in favor of activation of the vestibular system.

Additional evidence of activation of the vestibular system using our vestibular implant prototypes could be obtained by activation of the vestibulo-collic reflex demonstrated by the recording of cervical vestibular evoked myogenic potentials (cVEMPs). Cervical VEMPs are thought to reflect the functionality of the saccular part of the otolithic organ. ${ }^{64}$ Few groups reported successful measurements of cVEMPs elicited by electrical stimulation of the promontory in local anesthesia prior cochlear implantation ${ }^{65}$ or by direct electrical stimulation of the inferior vestibular nerve in patients undergoing a surgery of the cerebello-pontine angle in general anesthesia. ${ }^{66}$ In this second study there were no recordable cVEMPs when stimulating the superior vestibular nerve, which is in accordance with its probable saccular origin. In a recent pilot study conducted in 5 implanted patients, cVEMPs with its classical characteristics could be successfully elicited (see example in Figure 1.3) using a standard recording protocol, except for the stimulus, which consisted of 100 trials of single, cathodic-first, biphasic, and charge balanced pulses delivered at a rate of 5 pulses per second via the electrodes positioned in the lateral or the superior canals (intralabyrinthine). P1 and $\mathrm{N} 1$ latencies were in average $9.8 \mathrm{~s}( \pm 1.0 \mathrm{~s})$ and $16.9 \mathrm{~s}( \pm 1.7 \mathrm{~s})$, respectively. These findings indicate that it is possible to artificially restore the vetibulo-collic reflex in a patient with a complete bilateral vestibular loss and thus that useful motion information could potentially be delivered via the vestibulo-spinal pathways.

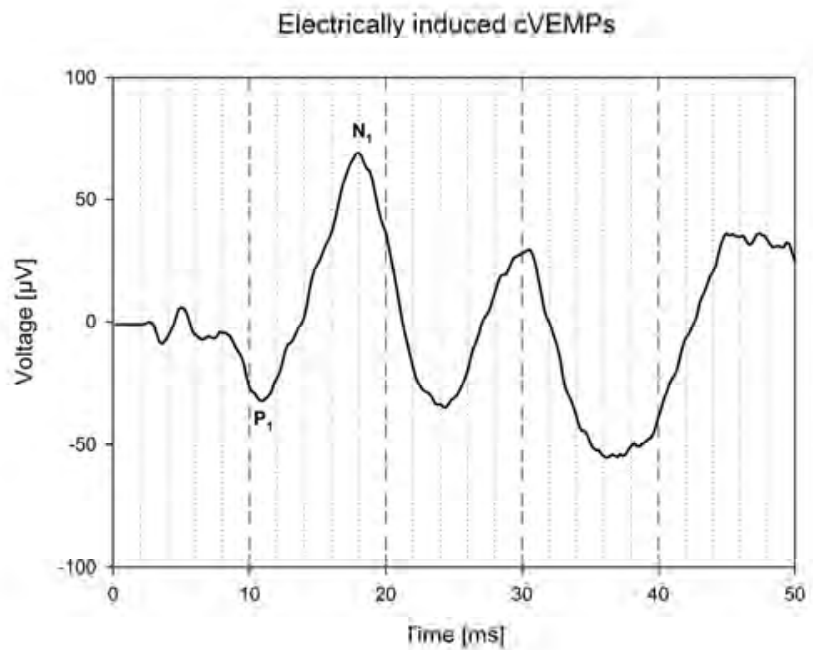

Figure 1.3 Morphology of cVEMPs recorded according to a standard protocol, except for the stimulus, which consisted of 100 trials of single cathodic first, biphasic, charge balanced pulses delivered at a rate of 5 pulses per second by the vestibular implant electrode positioned in the superior canal. 


\section{Discussion}

The Geneva-Maastricht team is the first (and practically the only one) to work with human patients fitted with a vestibular implant aimed at restoring vestibular function in cases of bilateral vestibular loss. Within the last 10 years, 13 patients have received single- and multi-channel vestibular implant prototypes. No medical complication has been observed. It is worth mentioning that, except for one patient who has normal hearing on the controlateral ear, all patients benefit fully from their cochlear implant and are fully satisfied with the device.

We have demonstrated that vestibular reflexes can be at least partially restored using a vestibular implant prototype. Besides the extensively studied VOR, here we show that the vestibulo-collic reflex can also be successfully activated. Average latencies of the cVEMPs response were however substantially shorter than those classically described with acoustic stimuli (P1=13ms/N1=23ms). Similar short latency cVEMP responses have been recently reported using electrical stimuli delivered with cochlear implants. ${ }^{67}$ These results could be explained by the fact that electrical stimulation directly activates the vestibular afferents, thus bypassing the mechanoelectrical transduction. However, the precise origin of these responses (canal afferents, otolithic afferents or a combination of both) still remains unclear. Finally, in order to evaluate whether the restoration of vestibular reflexes could be useful in a task representative of every-day difficulties faced by patients, we implemented a simple dynamic visual acuity protocol that could be performed with and without activation of the vestibular implant. ${ }^{26}$ The pilot results are striking, demonstrating that the implant significantly improves the ability to stabilize gaze in a dynamic condition such as walking. Altogether, these results represent a major step towards a useful device potentially addressing several major complaints of patients suffering with a bilateral vestibular loss (e.g., oscillopsia, postural control) and open the door for a clinical application in the near future.

Another research team reported results obtained in patients implanted with a modified cochlear implant with a trifurcating array incorporating 3 electrodes per array inserted into the ampullas. ${ }^{33,68}$ Their device was purely vestibular and did not have any cochlear electrodes. Their initial concept was also different from ours: their vestibular device was concieved to act as a pace maker that could be activated during a Menière's crisis to regulate vestibular function in patients suffering from intractable Menière's disease. Only one patient experienced a typical Menière's crisis after implantation. In this case, activation of the device led to a subjective improvement. Although this result is promising, the fact that no other Menière's crisis has been reported after implantation is compatible with the loss of the vestibular function observed after implantation in all 4 of them. Moreover the pre-operative residual hearing could not be preserved either in any of the 4 subjects participating in this study. Despite this negative results, in can not be ruled out that the principle of a 
specific vestibular pacemeker developed to control the fluctuation of the vestibular function in patients suffering handicapping Menière's disease, can still be a potential therapeutic application of the principle of electrical stimulation of the vestibular nerve.

\section{Future work}

As preservation of the vestibular and hearing function will be a crucial issue, substantial efforts are put into the design of new electrodes and surgical approaches to allow atraumatic implantation with preservation of the membraneous labyrinth. Particular attention is put into increasing the specificity of stimulation by optimally targetting the different branches of the vestibular nerve, inculding the saccular and utricular branches. Indeed canal and otolithic function are complementary and rehabilitation of both functions could be necessary to reach a higher level of functional rehabilitation.

The other main area of interest is improving and extending the rehabilitation prospects of the device, particularly regarding imbalance and postural control. Finally, in parallel to this fundamental clinical developments our current and future research will also be devoted to gathering fundamental knowledge on the vestibular system and its interactions with extra-vestibular mechanisms. Indeed, the vestibular implant offers the unique opportunity to selectively deliver a vestibular stimulus to the central nervous system. This opens an unprecedented door for the study of the integration and perception of vestibular input in the multisensory, multimodal system of balance. 


\section{References}

1. Miffon M,Guyot JP. Difficulties faced by patients suffering from total bilateral vestibular loss. ORL J Otorhinolaryngol Relat Spec 2015;77:241-7.

2. van de Berg $R, M$. van Tilburg $M$, Kingma $H$. Bilateral vestibular hypofunction: challenges in establishing the diagnosis in adults, ORL J Otorhinolaryngol Relat Spec 2015;77:197-218.

3. Guinand N, Boselie F, Guyot JP, Kingma H. Quality of life of patients with bilateral vestibulopathy. Ann Otol Rhinol Laryngol 2012;121:471-7.

4. Sun DQ, Ward BK, Semenov YR, Carey JP, Della Santina CC. Bilateral vestibular deficiency: quality of life and economic implications. JAMA Otolaryngol Head Neck Surg 2014;140:527-34.

5. Zingler VC, Cnyrim C, Jahn K, Weintz E, Fernbacher J, Frenzel C, Brandt T, Strupp M. Causative factors and epidemiology of bilateral vestibulopathy in 255 patients. Ann Neurol 2007;61:524-32.

6. Gillespie MB, Minor LB. Prognosis in bilateral vestibular hypofunction. Laryngoscope 1999;109:35-41.

7. Honegger F, Hillebrandt IM, van den Elzen NG, Tang KS, Allum JH. The effect of prosthetic feedback on the strategies and synergies used by vestibular loss subjects to control stance. J Neuroeng Rehabil 2013;10:115.

8. Janssen M, Stokroos R, Aarts J, van Lummel R, Kingma H. Salient and placebo vibrotactile feedback are equally effective in reducing sway in bilateral vestibular loss patients. Gait Posture 2010;31:213-7.

9. Ma CZ, Wan AH, Wong DW, Zheng YP, Lee WC. A vibrotactile and plantar force measurement-based biofeedback ystem: paving the way towards wearable balance-improving devices. Sensors (Basel) 2015;15:31709-22.

10. Chen W, Jongkamonwiwat N, Abbas L, Eshtan SJ, Johnson SL, Kuhn S, Milo M, Thurlow JK, Andrews PW, Marcotti W, Moore HD, Rivolta MN. Restoration of auditory evoked responses by human ES-cellderived otic progenitors. Nature 2012;490:278-82.

11. Dai C, Fridman GY, Davidovics NS, Chiang B, Ahn JH, Della Santina CC. Restoration of 3D vestibular sensation in rhesus monkeys using a multichannel vestibular prosthesis. Hear Res 2011;281:74-83.

12. Lewis RF, Haburcakova C, Gong W, Makary C, Merfeld DM. Vestibulo-ocular reflex adaptation investigated with chronic motion-modulated electrical stimulation of semicircular canal afferents. J Neurophysiol 2010;103:1066-79.

13. Perez Fornos A, Guinand N, van de Berg R, Stokroos R, Micera S, Kingma H, Pelizzone M, Guyot JP. Artificial balance: restoration of the vestibulo-ocular reflex in humans with a prototype vestibular neuroprosthesis. Front Neurol 2014;5:66.

14. Lopez C, Blanke O, The thalamocortical vestibular system in animals and humans. Brain Res Rev 2011; 67:119-46.

15. Merfeld DM, Zupan L, Peterka RJ. Humans use internal models to estimate gravity and linear acceleration. Nature 1999;398:615-8.

16. Chen G, King JA, Burgess N, O'Keefe J. How vision and movement combine in the hippocampal place code. Proc Natl Acad Sci U S A 2013;110:378-83.

17. Kandel e. A place and a grid in the sun. Cell 2014;159:1239-42.

18. Gotoh TM, Fujiki N, Matsuda T, Gao S, Morita H. Roles of baroreflex and vestibulosympathetic reflex in controlling arterial blood pressure during gravitational stress in conscious rats. Am J Physiol Regul Integr Comp Physiol 2004;286:R25-30.

19. Tanaka K, Abe C, Awazu C, Morita H. Vestibular system plays a significant role in arterial pressure control during head-up tilt in young subjects. Auton Neurosci 2009;148:90-6.

20. Aoki M, Sakaida Y, Tanaka K, Mizuta K, Ito Y. Evidence for vestibular dysfunction in orthostatic hypotension. Exp Brain Res 2012;217:251-9.

21. Woodring SF, Yates BJ. Responses of ventral respiratory group neurons of the cat to natural vestibular stimulation. Am J Physiol 1997;273:R1946-56.

22. Cordero L, Clark DL, Schott L. Effects of vestibular stimulation on sleep states in premature infants. Am J Perinatol 1986;3:319-24.

23. Fuller PM, Jones TA, Jones SM, Fuller CA. Evidence for macular gravity receptor modulation of hypothalamic, limbic and autonomic nuclei. Neuroscience 2004;129:461-71. 
24. Smith PF, Zheng Y. From ear to uncertainty: vestibular contributions to cognitive function. Front Integr Neurosci 2013;7:84.

25. Smith PF, Darlington CL. Personality changes in patients with vestibular dysfunction. Front Hum Neurosci 2013;7:678.

26. Guinand N, Pijnenburg M, Janssen M, Kingma H. Visual acuity while walking and oscillopsia severity in healthy subjects and patients with unilateral and bilateral vestibular function loss. Arch Otolaryngol Head Neck Surg 2012;38:301-6.

27. Lambert S, Sigrist A, Delaspre O, Pelizzone M, Guyot JP. Measurement of dynamic visual acuity in patients with vestibular areflexia. Acta Otolaryngol 2010;130:820-3.

28. Vital D, Hegemann SC, Straumann D, Bergamin O, Bockisch CJ, Angehrn D, Schmitt KU, Probst R. A new dynamic visual acuity test to assess peripheral vestibular function. Arch Otolaryngol Head Neck Surg 2010;136:686-91.

29. Priesol AJ, Valko Y, Merfeld DM, Lewis RF. Motion perception in patients with idiopathic bilateral vestibular hypofunction. Otolaryngol Head Neck Surg 2014;150:1040-2.

30. Kingma H, Gauchard GC, de Waele C, van Nechel C, Bisdorff A, Yelnik A, Magnusson M, Perrin PP. Stocktaking on the development of posturography for clinical use. J Vestib Res 2011;21:117-25.

31. Pelizzone M, Perez-Fornos A, Ranieri M, Cavuscens S. Device for electrical stimulation of neural and/or muscular tissue, has signal processing unit to transform input signal received from signal sensors into modulated electrical output signal to be treated by speech processor., in, vol WO2014118094-A1; EP2762196-A1, 2013.

32. Goldberg JM, Fernandez C. Physiology of peripheral neurons innervating semicircular canals of the squirrel monkey. I. Resting discharge and response to constant angular accelerations. J Neurophysiol 1971;34:635-60.

33. Golub JS, Ling L, Nie K, Nowack A, Shepherd SJ, Bierer SM, Jameyson E, Kaneko CR, Phillips JO, Rubinstein JT. Prosthetic implantation of the human vestibular system. Otol Neurotol 2014;35:136-47.

34. Gacek RR, Gacek MR. Results of singular neurectomy in the posterior ampullary recess. ORL J Otorhinolaryngol Relat Spec 2002;64:397-402.

35. Feigl G, Kos I, Anderhuber F, Guyot JP, Fasel J. Development of surgical skill with singular neurectomy using human cadaveric temporal bones. Ann Anat 2008;190:316-23.

36. Kos MI, Feigl G, Anderhuber F, Wall C, Fasel JH, Guyot JP. Transcanal approach to the singular nerve. Otol Neurotol 2006;27:542-6.

37. Feigl GC, Fasel JH, Anderhuber F, Ulz H, Rienmuller R, Guyot JP, Kos IM. Superior vestibular neurectomy: a novel transmeatal approach for a denervation of the superior and lateral semicircular canals. Otol Neurotol 2009;30:586-91.

38. Dai C, Fridman GY, Davidovics NS, Chiang B, Ahn JH, Della Santina CC. Restoration of 3D vestibular sensation in rhesus monkeys using a multichannel vestibular prosthesis. Hear Res 2011;281:74-83.

39. van de Berg R, Guinand N, Guyot JP, Kingma H, Stokroos RJ. The modified ampullar approach for vestibular implant surgery: feasibility and its first application in a human with a long-term vestibular loss. Front Neurol 2012;3:18.

40. Bierer SM, Ling L, Nie K, Fuchs AF, Kaneko CR, Oxford T, Nowack AL, Shepherd SJ, Rubinstein JT, Phillips JO. Auditory outcomes following implantation and electrical stimulation of the semicircular canals. Hear Res 2012;287:51-6.

41. Dai C, Fridman GY, Della Santina CC., Effects of vestibular prosthesis electrode implantation and stimulation on hearing in rhesus monkeys. Hear Res 2011;277:204-10.

42. Rubinstein JT, Bierer S, Kaneko C, Ling L, Nie K, Oxford T, Newlands S, Santos F, Risi F, Abbas PJ, Phillips JO. Implantation of the semicircular canals with preservation of hearing and rotational sensitivity: a vestibular neurostimulator suitable for clinical research. Otol Neurotol 2012;33:789-96.

43. Tang S, Melvin TA, Della Santina CC. Effects of semicircular canal electrode implantation on hearing in chinchillas, Acta Otolaryngol 2009;129:481-6.

44. Tran H, de Waele C, Beraneck M, Vassias I, Gioanni H, Huy PT, Herman P, Vidal PP, Kania RE. Auditory outcomes after implantation and electrical stimulation of the lateral ampullar nerve in guinea pig. Ear Hear 2012;33:118-23.

45. Agrawal, Parnes LS. Human experience with canal plugging. Ann N Y Acad Sci 2001;942:300-5. 
46. Charpiot A, Rohmer D, Gentine A. Lateral semicircular canal plugging in severe Meniere's disease: a clinical prospective study about 28 patients. Otol Neurotol 2010;31:237-40.

47. Limb CJ, Carey JP, Srireddy S, Minor LB. Auditory function in patients with surgically treated superior semicircular canal dehiscence. Otol Neurotol 2006;27:969-80.

48. Wall C 3rd, Kos MI, Guyot JP. Eye movements in response to electric stimulation of the human posterior ampullary nerve. Ann Otol Rhinol Laryngol 2007;116:369-74.

49. Guyot JP, Sigrist A, Pelizzone M, Feigl CC, Kos MI. Eye movements in response to electrical stimulation of the lateral and superior ampullary nerves. Ann Otol Rhinol Laryngol 2011;120:81-7.

50. Birns JW, Honrubia V. Effects of halothane on the vestibulo-ocular reflex. Am J Otolaryngol 1980; 1:418-25.

51. Nakao A, Markham CH, Curthoys IS. Modification of single neural elements in vestibular nystagmus by anesthesia. Acta Otolaryngol 1980;89:121-34.

52. Cavazzuti M, Porro CA, Barbieri A, Galetti A. Brain and spinal cord metabolic activity during propofol anaesthesia. Br J Anaesth 1991;66:490-5.

53. Janeke JB, Jongkees LB, Oosterveld WJ. Selective suppression of the fast phase of labyrinthine nystagmus by phentanyl (fentanyl). Acta Otolaryngol 1969;68:468-73.

54. Guinand N, Guyot JP, Kingma H, Kos I, Pelizzone M. Vestibular implants: The first steps in humans. Conf Proc IEEE Eng Med Biol Soc 2011 (2011):2262-4.

55. Merfeld DM, Gong W, Morrissey J, Saginaw M, Haburcakova C, Lewis RF. Acclimation to chronic constant-rate peripheral stimulation provided by a vestibular prosthesis. IEEE Trans Biomed Eng 2006; 53:2362-72.

56. Guyot JP, Sigrist A, Pelizzone M, Kos MI. Adaptation to steady-state electrical stimulation of the vestibular system in humans. Ann Otol Rhinol Laryngol 2011;120:143-9.

57. Guinand N, van de Berg R, Cavuscens S, Stokroos RJ, Ranieri M, Pelizzone M, Kingma H, Guyot JP, Perez-Fornos A. Vestibular implants: 8 years of experience with electrical stimulation of the vestibular nerve in 11 patients with bilateral vestibular loss. ORL J Otorhinolaryngol Relat Spec 2015;77:227-40.

58. van de Berg R, Guinand N, Nguyen K, Ranieri M, Cavuscens S, Guyot JP, Stokroos R, Kingma H, Perez Fornos A. The vestibular implant: Frequency-dependency of the electrically evoked Vestibulo-Ocular Reflex in humans. Frontiers in Systems Neuroscience 2015;8:255.

59. Guinand N, van de Berg R, Ranieri M, Cavuscens S, DiGiovanna J, Nguyen TA, Micera S, Stokroos R, Kingma H, Guyot JP, Pérez Fornos A. Vestibular implants: Hope for improving the quality of life of patients with bilateral vestibular loss. Conf Proc IEEE Eng Med Biol Soc 2015 (2015):7192-5.

60. Guinand N, van de Berg R, Pelizzone M, Stokroos R, Kingma H, Guyot JP, Perez Fornos A. Walking with the Geneva-Maastricht Vestibular Implant : normalization of dynamic visual acuity in patients with a bilateral vestibular loss. In: 38th Annual Midwinter Meeting, Association for Research in Otolaryngology, Baltimore, USA, 2015.

61. Lewis RF, Haburcakova C, Gong W, Lee D, Merfeld D. Electrical stimulation of semicircular canal afferents affects the perception of head orientation. J Neurosci 2013;33:9530-5.

62. Lewis RF, Haburcakova C, Merfeld DM. Tilt psychophysics measured in nonhuman primates. Ann N Y Acad Sci 2005;1039:294-305.

63. Lewis RF, Haburcakova C, Merfeld DM. Roll tilt psychophysics in rhesus monkeys during vestibular and visual stimulation. J Neurophysiol 2008;100:140-53.

64. Rosengren SM, Welgampola MS, Colebatch JG. Vestibular evoked myogenic potentials: past, present and future. Clin Neurophysiol 2010;121:636-51.

65. Park JJ, Shen A, Westhofen M. Promontory electrical stimulation to elicit vestibular evoked myogenic potentials (VEMPs). Acta Otolaryngol 2015;135:239-45.

66. Basta D, Todt I, Eisenschenk A, Ernst A. Vestibular evoked myogenic potentials induced by intraoperative electrical stimulation of the human inferior vestibular nerve. Hear Res 2005;204:111-4.

67. Parkes WJ, Gnanasegaram JJ, Cushing SL, McKnight CL, Papsin BC, Gordon KA. Vestibular evoked myogenic potential testing as an objective measure of vestibular stimulation with cochlear implants. The Laryngoscope (2016), n/a-n/a. 
68. Phillips JO, Ling L, Nie K, Jameyson E, Phillips CM, Nowack AL, Golub JS, Rubinstein JT. Vestibular implantation and longitudinal electrical stimulation of the semicircular canal afferents in human subjects. J Neurophysiol 2015;113:3866-92. 



\section{Chapter 2}

\section{Quality of life of patients with bilateral vestibulopathy}

Nils Guinand, Frans Boselie, Jean-Philippe Guyot, Herman Kingma Annals of Otology, Rhinology \& Laryngology 2012;121(7):471-477 


\section{Abstract}

\section{Objectives}

Currently, there is no evidence of an effective treatment for patients with bilateral vestibulopathy (BV). Their main complaints are oscillopsia and imbalance. Opinions about the impact of BV on their quality of life are controversial, and their handicap is not always recognized, even among otoneurologists. The aim of this study was to objectively assess the health status of BV patients in order to evaluate the need for pursuing efforts toward the development of new treatments.

\section{Methods}

The Short-Form Health Survey (SF-36), the Dizziness Handicap Inventory (DHI), the Short Falls Efficacy Scale-International (Short FES-I), and an oscillopsia severity questionnaire were submitted to $39 \mathrm{BV}$ patients. The SF-36 scores were compared to the scores of a general Dutch population. The DHI scores were correlated to the oscillopsia severity scores. The Short FES-I scores were compared to scores in an elderly population. Residual otolithic function was correlated to all scores, and hearing to SF-36 scores.

\section{Results}

Compared to the general Dutch population, the BV patients scored significantly worse on the "physical functioning," "role physical," "general health," "vitality," and "social functioning" SF-36 variables $(P<0.05)$. The DHI scores were strongly correlated with the oscillopsia severity scores $(r=0.75 ; P<0.000001)$. The Short FES-I scores indicated a slight to moderate increase in the patients' fear of falling. No significant score differences were found between BV patients with residual otolithic function and patients with complete BV. There was no correlation between hearing status and SF-36 scores.

\section{Conclusions}

The results correlate with our clinical impression that BV has a strong negative impact on physical and social functioning, leading to a quality-of-life deterioration. There is a clear need for a therapeutic solution. Efforts toward the development of a vestibular implant are justified. 


\section{Introduction}

The vestibular system enables automatization of balance control, automatization of spatial orientation, and automatization of image stabilization during head movements, allowing the simultaneous performance of various cognitive tasks. For example, under normal conditions, one of the main tasks of the vestibulo-ocular reflex (VOR) is to automatically execute adequate compensatory eye movements during head movements. The semicircular canals' system registers 3-dimensional (3-D) angular accelerations and provides the central nervous system with information about angular head velocity via the vestibular nerve. In case of a bilateral failure of the system, the angular VOR can no longer be elicited. The subject is therefore no longer able to maintain sharp vision on a fixed target during high-frequency head movements, having oscillopsia and reduced visual acuity. It has recently been shown that it is possible to partially restore the angular VOR in animals. ${ }^{1}$ In humans, it is possible to control eye movements by selective acute electric stimulation of the vestibular nerve branches. Chronic electric stimulation of the ampullary nerves allows restoration of a baseline firing rate that can be modulated to generate controlled eye movements. ${ }^{2-6}$ Those major findings indicate the feasibility of restoring an angular VOR with a vestibular implant that would artificially register 3-D angular accelerations by use of gyroscopes and accelerometers, process the information, and transmit it to the brain via electrodes implanted in the vicinity of the vestibular nerve branches in a way similar to that of a cochlear implant. Patients with bilateral vestibulopathy (BV) would be first-choice candidates. Bilateral vestibulopathy is a chronic condition of which the causes can be ototoxic, infectious, traumatic, autoimmune, or congenital, but in approximately $50 \%$ of cases, no cause can be found. ${ }^{7}$ Currently, there is no clear evidence of an effective treatment for BV patients, and in the long term there is no improvement of the condition for the majority of them, despite vestibular exercises and balance training. ${ }^{8}$ The prevalence of BV has been estimated at 81 in 100,000 persons, corresponding to 500,000 patients in Europe and the United States and as many as 3 million worldwide. ${ }^{9}$ With progressive aging of the populations of developed countries, presby-vestibulopathy, a progressive neurosensory degeneration that is generally bilateral, could affect an increasing number of patients in the future. ${ }^{10}$ Patients with BV complain mainly of oscillopsia (a sensation that the visual environment is moving when it is not) and of imbalance. Symptoms related to vestibular function impairment, such as vertigo, dizziness, headache, nausea, double vision, photophobia, ataxia, and light-headedness, have also been reported in BV patients. $^{11,12}$ Those symptoms were rarely mentioned spontaneously by our BV patients and were very heterogeneous among them. Moreover, it can be difficult to accurately define those symptoms. We therefore decided to focus mainly on oscillopsia. Additionally, BV can also induce neuropsychological modifications of body perception in space. ${ }^{13}$ As to how BV affects patients' quality of life, otoneurologists 
differ greatly. Some would say that BV patients adapt very well to the absence of vestibular cues by relying mainly on visual and proprioceptive information and by an increase of their tolerance to oscillopsia, ${ }^{14}$ undergoing a minor impact on their quality of life. Our experience tends to show the opposite. Indeed, most of our BV patients mention a decrease of their physical performance, and also complain that their condition is not understood and acknowledged by others. The data on BV patients' quality of life are sparse and are limited to a small number of patients.

Health-related quality-of-life measurements are widely used to assess the burden of a chronic disease, to compare treatments, and to evaluate the efficacy of treatment. They could also be useful tools for justifying the development of new treatments by providing a more objective evaluation of a disease's impact on patients' quality of life. A multidimensional approach, combining a validated general health survey and a validated disease-specific questionnaire, has been chosen to assess the impact of BV on patients' lives. As there is not any standardized way to evaluate oscillopsia, a new questionnaire was developed. The aim of this study was to assess the quality of life, the self-perceived handicap, the "fear of falling," and the severity of oscillopsia in BV patients. As moderate and severe bilateral hearing impairments have a significant impact on general health survey scores, ${ }^{15}$ audiometric evaluation was systematically performed. The causes of bilateral vestibular function loss were documented.

\section{Materials and methods}

\section{BV patients}

Thirty-nine BV patients (22 male and 17 female; mean age, 57 years; age range, 29 to 83 years) who were investigated at the Division of Balance Disorders at Maastricht University Hospital between October 2009 and April 2011 were prospectively included in the study (Table 2.1). A search of the 2006 to 2009 (January 2006 to September 2009) department database was performed with the first criteria listed below. Eightyone subjects were found. Of them, 48 accepted our invitation. The remaining 33 could not be reached or declined our invitation. Of those 48 patients, 25 fulfilled the second and third criteria listed below and were included in the study. Fourteen BV patients had diagnoses made between October 2009 and April 2011 and fulfilled all 3 inclusion criteria.

The 3 inclusion criteria were 1) mean peak slow phase velocity of no more than $5 \% \mathrm{~s}$ in bilateral bithermal caloric irrigations, 2) gain of less than $15 \%$ on rotatory chair tests, and 3) pathological head impulse test results for the horizontal and vertical canals. Electronystagmography was used for vestibular testing. Bilateral bithermal $\left(30^{\circ} \mathrm{C}\right.$ and $44^{\circ} \mathrm{C}$ ) caloric irrigations with water were performed by experienced technicians under standard conditions. Rotatory chair tests consisted of horizontal and vertical torsion 
swings $\left(0.11 \mathrm{~Hz}\right.$; wmax of $\left.100^{\circ} / \mathrm{s}\right)$ and bilateral velocity steps ( $\omega$ of $\left.250^{\circ} / \mathrm{s}\right)$. Manual head impulse test results were recorded with a high-speed camera (Casio Exilim, Pro EX-F1, 12. optical zoom, high speed camera, 300 frames per second) in the 3 semicircular canal planes. Presence of correction saccades in all 6 directions was considered as pathological. All recordings were analyzed by an experienced otolaryngologist.

Table 2.1 Demographic variables in BV patients and in general Dutch population.

\begin{tabular}{lcc}
\hline & $\begin{array}{c}\text { BV-patietns } \\
(\mathrm{N}=39)\end{array}$ & $\begin{array}{c}\text { Normal population } \\
(\mathrm{N}=1742)\end{array}$ \\
\hline Age & & \\
mean (SD) & $57.0(10.9)$ & $47.6(18.0)$ \\
range & $29-83$ & $16-94$ \\
Gender & & \\
male & $56 \%$ & $56 \%$ \\
female & $44 \%$ & $44 \%$ \\
\hline
\end{tabular}

Assessment of the otolithic function consisted of cervical vestibular evoked myogenic potentials (cVEMPs; Nicolet, Viking IIIP). The active electrodes were placed on the main belly of the sternocleidomastoid muscle, equidistant from the mastoid process and the sternum. The reference electrodes were placed on the superior part of the sternum, and the ground electrode was placed on the forehead. The patients were in the supine position; they were instructed to turn the head contralateral to the stimulation side and then to lift it in order to contract the sternocleidomastoid muscle ipsilateral to the stimulation. The electromyographic results were recorded, amplified, and rectified. Tone bursts $(500 \mathrm{~Hz}$ at $130 \mathrm{~dB}$ sound pressure level stimulus repetition rate of $5.1 \mathrm{~Hz}$, rise time of $2 \mathrm{~ms}$, plateau of $2 \mathrm{~ms}$, and fall time of $2 \mathrm{~ms}$ ) were transmitted unilaterally via headphones to the right and left ears. Signals of 150 stimuli were averaged. Cervical vestibular evoked myogenic potentials were considered present when unilateral or bilateral positive (P1) and negative (N2) peaks could be identified. The presence of cVEMPs indicated at least a residual otolithic function, of saccular origin. ${ }^{16}$ Patients with bilateral absence of cVEMPs and the presence of an air-bone gap were omitted from the calculation.

The hearing assessment consisted of a pure tone air and bone conduction audiogram. Average air conduction thresholds of more than $25 \mathrm{~dB}$ hearing level $(\mathrm{HL})$ at the frequencies $0.5,1,2$, and $4 \mathrm{kHz}$ were considered to show hearing impairment. Thresholds between 26 and $45 \mathrm{~dB}$ HL were defined as mild hearing loss, thresholds of $46 \mathrm{~dB}$ to $65 \mathrm{~dB} \mathrm{HL}$ as moderate loss, and thresholds above $65 \mathrm{~dB} \mathrm{HL}$ as severe loss. The level of hearing loss was defined according to the best ear in patients with bilateral impairment, and according to the worst ear for patients with unilateral impairment. 
All patients had a neuro-ophthalmologic examination. The oculomotor function was normal in all BV patients. Polyneuropathy was reported in 1 BV patient. All patients were investigated on a volunteer basis and gave their written informed consent. The investigations were conducted according to the principles expressed in the Declaration of Helsinki.

\section{Questionnaires}

The questionnaires were submitted to patients during an interview at the Division of Balance Disorders at Maastricht University Hospital.

SF-36. The Short-Form Health Survey (SF-36) is a validated short-form health questionnaire composed of 36 items grouped into 8 variables: "physical functioning," "role physical," "bodily pain," "general health," "vitality," "social functioning," "role emotional," and "mental health," assessing both mental and physical health. ${ }^{17,18}$ For each variable, item scores are coded, summed, and transformed on a scale from 0 (worst possible health state measured by the questionnaire) to 100 (best possible health state). It is a generic health measure, rather than disease-specific. The validated Dutch version of the SF-36 was used in the present study. ${ }^{19}$ The SF-36 scores of the BV patients were compared to scores of a general Dutch population.

DHI. The Dizziness Handicap Inventory (DHI) is a validated 25-item, self-perceived handicap scale designed to assess the effect of dizziness and unsteadiness on quality of life. The items are grouped into 3 subscales evaluating the effects of dizziness and unsteadiness on emotional, functional, and physical aspects of daily living. For each item, there are 3 possible answers: no, sometimes, and yes, giving, respectively, 0, 2, and 4 points. A total score of 100 corresponds to the worst self-perceived disability handicap, and a total score of 0 to an absence of handicap. ${ }^{20}$ As it might not be adequate to use the 3 subscales separately, only the total $\mathrm{DHI}$ score was used for interpretation. ${ }^{21,22}$

Short FES-I. The Short Falls Efficacy Scale-International (Short FES-I) is a validated questionnaire. It has been implemented to evaluate the fear of falling, especially in elderly persons. ${ }^{23}$

Oscillopsia Severity Questionnaire. A questionnaire assessing oscillopsia severity was developed for the purpose of the study. Oscillopsia was defined as a "sensation that the visual environment is moving when it's not." The 9 questionnaire items investigated oscillopsia frequency in different situations encountered in daily life (Table 2.2).

Each item was scored 1 (never), 2 (seldom), 3 (sometimes), 4 (often), or 5 (always). The mean scores gave an oscillopsia severity score ranging from 1 to 5 . Mean scores of 3 or more were considered as indicating moderate to extreme oscillopsia severity. The internal consistency of the questionnaire was measured with Cronbach's $\alpha$. The coefficient $\alpha$ would be 1 if the questionnaire items were perfectly correlated, and 0 if 
they were independent. Cronbach's $\alpha$ was 0.88 in the group of BV patients. Values above 0.7 are generally considered acceptable. ${ }^{24}$

Table 2.2 Oscillopsia severity questionnaire.

1. Do you have the sensation that the visual environment is moving when it's not?

2. By dim light, do you have the sensation that what you see is not stable?

3. Is it difficult for you to recognize known faces when you are walking?

4. When you are reading, do you have the sensation that the text is not stable?

5. When you are watching television, do you have the sensation that the image is not stable?

6. When you are driving your car, do you have the sensation that what you see is not stable?

7. As a car passenger, do you have the sensation that what you see is not stable?

8. When you are riding bicycle, do you have the sensation that what you see is not stable?

9. When you are walking on uneven ground, do you have the sensation that what you see is not stable?

always $=5$, often $=4$, sometimes $=3$, seldom $=2$, never $=1$

\section{Statistical analysis}

Means and standard errors were obtained for all SF-36 variables. For each variable, expected SF-36 scores were computed from age- and sex-specific equations obtained in the general population, provided by Aaronson et al. ${ }^{19}$ (also Aaronson, personal communication, 2010). The differences between actual and expected scores were tested by means of 2 -tailed paired $t$-tests. A Mann-Whitney $U$ test was used to compare SF-36 scores, DHI scores, and oscillopsia severity scores between BV patients with and without residual otolithic function. A Mann-Whitney $U$ test was further used to compare the SF-36 scores of BV patients with moderate or severe bilateral hearing loss with the SF-36 scores of BV patients with mild bilateral hearing loss, unilateral hearing loss, or normal hearing. The Pearson product-moment correlation coefficient between DHI scores and oscillopsia severity scores was determined. For all tests, statistical significance was defined as a $P$ value of no more than 0.05 .

\section{Results}

The cause of bilateral vestibular function loss was ototoxicity in 7 patients $(17.96 \%$; due to systemic gentamicin treatment in 6 and cisplatin in 1), related to a COCH gene mutation (DFNA9) in $4(10.26 \%)$, and infectious in 3 ( 2 patients or $5.13 \%$ with positive serologic tests for Borrelia burgdorferi and 1 patient or $2.56 \%$ who had meningitis at the age of 1 year). One patient (2.56\%) reported the first symptoms to have occurred after a severe head trauma without fracture (a fall on stairs). One patient (2.56\%) had bilateral Meniere's disease, and in 4 patients (10.26\%), a positive family history for inner ear disease was known. One patient $(2.56 \%)$ was known to have Wegener's granulomatosis. In 18 patients (46.15\%), no cause could be found (Table 2.3 ). 
Table 2.3 Causes of BV.

\begin{tabular}{lcc}
\hline Etiology & $\mathrm{n}(\mathrm{N}=39)$ & $\%$ \\
\hline Idiopathic & 18 & 46.15 \\
Ototoxic & 7 & 17.96 \\
Positive family history for inner ear disease & 4 & 10.26 \\
COCH gene mutation (DFNA9) & 4 & 10.26 \\
Infectious & 3 & 7.69 \\
Morbus Wegener & 1 & 2.56 \\
Ménière disease & 1 & 2.56 \\
Traumatic & 1 & 2.56 \\
\hline
\end{tabular}

Five of the 8 SF-36 variable scores were significantly lower $(P<0.05)$ than predicted, adjusted scores from the general Dutch population. The 5 SF-36 variables concerned were "physical functioning," "role physical," "general health," "vitality," and "social functioning." There was no significant difference for the other 3 SF-36 variables $(P>0.05$; Table 2.4). The presence of cVEMPs could be identified in 20 patients. Two patients with audiometric tests showing an air-bone gap of more than $10 \mathrm{~dB}$ over 0.5 , 1, 2, and $4 \mathrm{~Hz}$ and absent cVEMPs were not included for calculation. The SF-36 scores, $\mathrm{DHI}$ scores, and oscillopsia severity scores were not significantly different between BV patients with residual otolithic function and BV patients without residual otolithic function $(P>0.05)$.

Table 2.4 SF-36 scores of BV patients compared to predicted scores, computed from age- and sexspecific equations obtained in general Dutch population.

\begin{tabular}{lccc}
\hline SF-36 variables & BV-patients $(\mathrm{n}=39)$ & $\begin{array}{c}\text { Normal Population ( } \mathrm{n}=1742) \\
\text { predicted scores } \\
\text { mean (standard error) }\end{array}$ & $P$ \\
\hline Physical functioning & $54.7(4.3)$ & $76.8(1.2)$ & 0.000 \\
Role physical & $51.9(6.2)$ & $71.3(1.1)$ & 0.005 \\
Body pain & $76.5(3.8)$ & $72.1(0.7)$ & 0.267 \\
General Health & $57.5(3.5)$ & $67.0(0.7)$ & 0.014 \\
Vitality & $57.1(2.8)$ & $67.1(0.7)$ & 0.003 \\
Social functioning & $67.3(3.9)$ & $81.9(0.5)$ & 0.001 \\
Role emotional & $75.2(5.4)$ & $80.7(0.6)$ & 0.342 \\
Mental health & $72.1(2.4)$ & $75.7(0.5)$ & 0.174 \\
\hline
\end{tabular}

The $\mathrm{DHI}$ scores were strongly correlated to the oscillopsia severity scores $(r=0.75$; $P<0.000001)$. DHI scores from 30 to 60 indicate a moderate selfperceived handicap, and scores above 60 indicate a severe handicap. ${ }^{25}$ Thirty-three patients (85\%) had a score of 30 or above, and 17 patients (44\%) had a score of 60 or more (see Figure 2.1). 


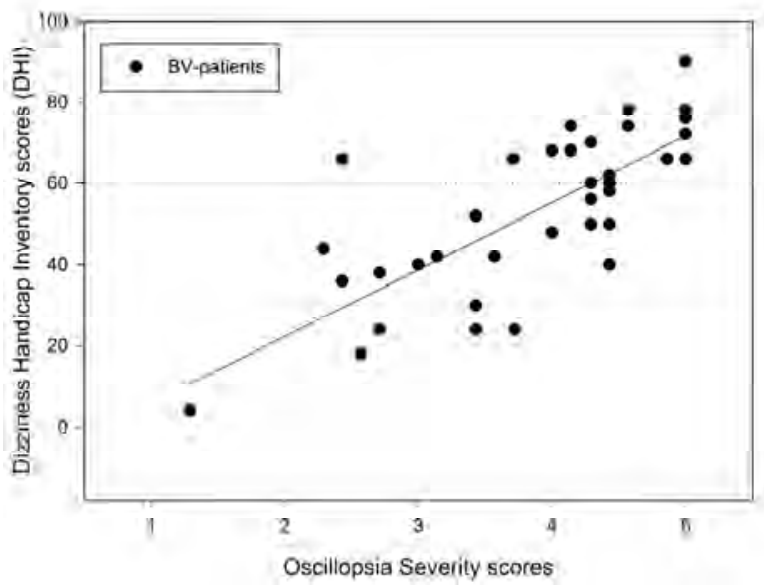

Figure 2.1 Dizziness Handicap Inventory (DHI) scores versus escillopsia severity scores in patients with bilateral vestibulopathy. Self-perceived handicap is considered moderate for DHI scores between 30 and 60, and severe for DHI scores above 60 .

The Short FES-I scores corresponded to a slight to moderate fear of falling for 29 of 39 patients (74\%). Three patients (8\%) had severe fear of falling, and $7(18 \%)$ had no fear of falling ${ }^{23}$ (Table 2.5).

Table 2.5 Short FES-I scores of BV patients.

\begin{tabular}{lc}
\hline Fear to fall & BV-Patients (N=39) \\
\hline No & 7 \\
Slight & 12 \\
moderate & 17 \\
Severe & 3 \\
\hline
\end{tabular}

A pure tone audiogram was performed in 38 of the 39 patients. A bilateral hearing impairment was found in 17 patients (44.7\%). Among them, 4 (10.5\% of 38 patients) had mild hearing loss, 3 (7.9\%) moderate, and 10 (26.3\%) severe. Unilateral hearing loss was present in 6 patients (15.8\%), and 15 (39.5\%) had normal hearing (Table 2.6). No significant differences in the SF-36 scores were found between BV patients with moderate or severe bilateral hearing loss and BV patients with mild bilateral hearing loss, unilateral hearing loss, or normal hearing $(P>0.05)$. 
Table 2.6 Audiometric findings in BV patients.

\begin{tabular}{lc}
\hline & BV-patients $(\mathrm{N}=38)$ \\
\hline bilateral hearing loss & \\
mild $(26-45 \mathrm{~dB} H \mathrm{~L})$ & $4(10.5 \%)$ \\
moderate $(46-65 \mathrm{~dB} \mathrm{HL})$ & $3(7.9 \%)$ \\
severe $(>65 \mathrm{~dB} \mathrm{HL})$ & $10(26.3 \%)$ \\
unilateral hearing loss $(>25 \mathrm{~dB} \mathrm{HL})$ & $6(15.8 \%)$ \\
normal hearing $(<25 \mathrm{~dB} \mathrm{HL})$ & $15(39.5 \%)$ \\
\hline
\end{tabular}

\section{Discussion}

The SF-36 scores correlate quite well with our clinical impression. Patients with BV progressively reduce their physical activities. In the Netherlands, a country with about 16 million inhabitants, over 14 million persons ride a bicycle every day, to go to work or social events. Not being able to ride a bicycle anymore is perceived as an important handicap. Most of our BV patients are affected; as a consequence, they have to modify their professional and social activities. As we learned from the different questionnaires, most of our BV patients also have important difficulties with simple tasks such as taking a shower, taking care of the household, going up stairs, or putting on clothes. The scores obtained for "physical functioning" and "social functioning" correlate very well with those observations. The "vitality" variable reveals that they feel less enthusiastic and energetic and are more often tired. The "general health" variable indicates that the BV patients perceive their health as worse than the health of most persons living in their social environment, and that they are not optimistic about improvement of their condition in the future.

Residual otolithic function of saccular origin was found in 20 BV patients (51\%). In contrast, in a study including 84 BV patients, of whom only $48 \%$ showed "complete" BV, cVEMPs were present in all patients, with bilateral pathological amplitudes in $61 \%$ of the cases. ${ }^{26}$ This difference could be explained by the fact that the criteria for defining BV patients differed. In our study, the inclusion criteria were stricter regarding semicircular canal function, which might be associated with poorer otolithic function. No significant score differences (SF-36, DHI, and oscillopsia severity) were found between BV patients with residual otolithic function and BV patients without identifiable otolithic function. This finding is in accordance with a study including 5 BV patients with preserved saccular function who reported oscillopsia while walking. ${ }^{27}$

The results indicate that $34.2 \%$ of the BV patients had moderate to severe bilateral hearing impairment. The SF-36 scores of the BV patients with moderate or severe bilateral hearing loss and the BV patients with mild bilateral hearing loss, unilateral hearing loss, or normal hearing were not significantly different, indicating that hearing level was not a relevant factor among our BV patients. The prevalence $(44.7 \%)$ of 
bilateral hearing impairment among the BV patients in this study is at least twice as high as that reported for an age-matched general population. ${ }^{15}$ Normal hearing was found in $39.5 \%$ of BV patients; this percentage is slightly above the $34 \%$ reported in $50 \mathrm{BV}$ patients by Rinne et al. ${ }^{11}$ and the $27 \%$ reported by Vibert et al. ${ }^{28}$ in a study of 52 BV patients. In a large study including 255 BV patients, based on bedside hearing tests and subjective hypoacusis, audiometry was only performed in 80 BV patients, and hearing loss was found in 78 of them. ${ }^{8}$ Most likely, no significant hearing loss would have been found in the remaining two thirds of BV patients. As the definition of $B V$ is not uniform and the hearing testing differs in different studies, interpretation of those results is limited. Interestingly, all 5 BV patients with an ototoxic cause due to gentamicin who completed audiometry had normal hearing. This finding is probably due to a higher gentamicin sensitivity on the part of the vestibular hair cells compared to the cochlear hair cells. ${ }^{29}$

The strong correlation with the DHI scores shows that the oscillopsia severity questionnaire can be used to assess oscillopsia severity in BV patients. The $\mathrm{DHI}$ scores indicate that the handicap was perceived as severe by $17 \mathrm{BV}$ patients (44\%) and as moderate by 16 (41\%). It is interesting to see that in a study of 19 patients with superior semicircular canal dehiscence, the mean DHI score was $44 .{ }^{30}$ Leaving patients with such a handicap without treatment was not even an option; indeed, they all benefited from surgical treatment with a middle fossa approach despite potential severe complications. ${ }^{30}$ In our BV patient group, 26 patients (67\%) had a DHI score higher than 44, without having any effective treatment option. Likewise, for most subjects in a group of patients with unilateral vestibular loss with a mean DHI score of 40 , there were effective medical, surgical, or rehabilitative treatments available. ${ }^{25}$ This finding shows clearly the need to propose therapeutic solutions to BV patients.

Patients with BV can modify their activities and the way they function according to symptom severity, preventing falls efficiently. Of 39 BV patients, only 3 (8\%) mentioned having fallen sporadically (results not documented). Fear of falling can be maintained at a low or moderate level, but at a cost, as indicated clearly by the negative impact on quality of life and patients' high ratings of self-perceived handicap. Efforts to develop a vestibular implant to restore the vestibular reflexes are worthwhile to pursue in order to propose a potential therapeutic solution to BV patients in the future. The SF-36, DHI, Short FES-I, and oscillopsia severity questionnaire could be very useful tools for monitoring BV patients after vestibular implantation. 


\section{References}

1. Lewis RF, Haburcakova C, Gong W, Makary C, Merfeld DM. Vestibuloocular reflex adaptation investigated with chronic motion-modulated electrical stimulation of semicircular canal afferents. J Neurophysiol 2010;103:1066-79.

2. Kos MI, Feigl G, Anderhuber F, Wall C, Fasel JH, Guyot JP. Transcanal approach to the singular nerve. Otol Neurotol 2006;27:542-6.

3. Feigl GC, Fasel JH, Anderhuber F, et al. Superior vestibular neurectomy: a novel transmeatal approach for a denervation of the superior and lateral semicircular canals. Otol Neurotol 2009;30:586-91.

4. Wall C III, Kos MI, Guyot JP. Eye movements in response to electric stimulation of the human posterior ampullary nerve. Ann Otol Rhinol Laryngol 2007;116:369-74.

5. Guyot JP, Sigrist A, Pelizzone M, Kos MI. Adaptation to steady-state electrical stimulation of the vestibular system in humans. Ann Otol Rhinol Laryngol 2011;120:143-9.

6. Guyot JP, Sigrist A, Pelizzone M, Feigl GC, Kos MI. Eye movements in response to electrical stimulation of the lateral and superior ampullary nerves. Ann Otol Rhinol Laryngol 2011;120:81-7.

7. Zingler VC, Weintz E, Jahn K, et al. Follow-up of vestibular function in bilateral vestibulopathy. J Neurol Neurosurg Psychiatry 2008;79:284-8.

8. Zingler VC, Cnyrim C, Jahn K, et al. Causative factors and epidemiology of bilateral vestibulopathy in 255 patients. Ann Neurol 2007;61:524-32.

9. Della Santina CC, Hoffman H. Prevalence and impact of bilateral vestibular deficiency (BVD): results from the 2008 United States National Health Interview Survey [Abstract]. J Vestib Res 2010;20:244-5.

10. Furman JM, Raz Y, Whitney SL. Geriatric vestibulopathy assessment and management. Curr Opin Otolaryngol Head Neck Surg 2010;18:386-91.

11. Rinne $T$, Bronstein AM, Rudge P, Gresty MA, Luxon LM. Bilateral loss of vestibular function: clinical findings in 53 patients. J Neurol 1998;245:314-21.

12. Zingler VC, Weintz E, Jahn K, et al. Causative factors, epidemiology, and follow-up of bilateral vestibulopathy. Ann N Y Acad Sci 2009;1164:505-8.

13. Grabherr L, Karmali F, Bach S, Indermaur K, Metzler S, Mast FW. Mental own-body and body-part transformations in microgravity. J Vestib Res 2007;17:279-87.

14. Grunfeld EA, Morland AB, Bronstein AM, Gresty MA. Adaptation to oscillopsia: a psychophysical and questionnaire investigation. Brain 2000;123:277-90.

15. Chia EM, Wang JJ, Rochtchina E, Cumming RR, Newall P, Mitchell P. Hearing impairment and healthrelated quality of life: the Blue Mountains Hearing Study. Ear Hear 2007;28:187-95.

16. Curthoys IS. A critical review of the neurophysiological evidence underlying clinical vestibular testing using sound, vibration and galvanic stimuli. Clin Neurophysiol 2010;121:132-44.

17. Ware JE Jr, Sherbourne CD. The MOS 36-item shortform health survey (SF-36). I. Conceptual framework and item selection. Med Care 1992;30:473-83.

18. Jenkinson C, Coulter A, Wright L. Short form 36 (SF36) health survey questionnaire: normative data for adults of working age. BMJ 1993;306:1437-40.

19. Aaronson NK, Muller M, Cohen PD, et al. Translation, validation, and norming of the Dutch language version of the SF-36 Health Survey in community and chronic disease populations. J Clin Epidemiol 1998;51:1055-68.

20. Jacobson GP, Newman CW. The development of the Dizziness Handicap Inventory. Arch Otolaryngol Head Neck Surg 1990;116:424-7.

21. Kurre A, Bastiaenen CH, van Gool CJ, Gloor-Juzi T, de Bruin ED, Straumann D. Exploratory factor analysis of the Dizziness Handicap Inventory (German version). BMC Ear Nose Throat Disord 2010;10:3.

22. Tamber AL, Wilhelmsen KT, Strand LI. Measurement properties of the Dizziness Handicap Inventory by cross-sectional and longitudinal designs. Health Qual Life Outcomes 2009;7:101.

23. Kempen GI, Zijlstra GA, van Haastregt JC. The assessment of fear of falling with the Falls Efficacy Scale-International (FES-I). Development and psychometric properties in Dutch elderly [in Dutch]. Tijdschr Gerontol Geriatr 2007;38:204-12. 
24. Nunnally JC. Psychometric theory. 2nd ed. New York, NY: McGraw-Hill, 1978.

25. Jacobson GP, Calder JH. Self-perceived balance disability/handicap in the presence of bilateral peripheral vestibular system impairment. J Am Acad Audiol 2000;11:76-83.

26. Zingler VC, Weintz E, Jahn K, et al. Saccular function less affected than canal function in bilateral vestibulopathy. J Neurol 2008;255:1332-6.

27. Brantberg K, Löfqvist L. Preserved vestibular evoked myogenic potentials (VEMP) in some patients with walkinginduced oscillopsia due to bilateral vestibulopathy. J Vestib Res 2007;17:33-8.

28. Vibert D, Liard P, Häusler R. Bilateral idiopathic loss of peripheral vestibular function with normal hearing. Acta Otolaryngol 1995;115:611-5.

29. Webster JC, McGee TM, Carroll R, Benitez JT, Williams ML. Ototoxicity of gentamicin. Histopathologic and functional results in the cat. Trans Am Acad Ophthalmol Otolaryngol 1970;74:1155-65.

30. Crane BT, Minor LB, Carey JP. Superior canal dehiscence plugging reduces dizziness handicap. Laryngoscope 2008;118:1809-13. 



\section{Chapter 3}

\section{Visual acuity while walking and oscillopsia severity in healthy subjects and patients with unilateral and bilateral vestibular function loss}

Nils Guinand, Mark Pijnenburg, Maurice Janssen, Herman Kingma Arch Otolaryngol Head Neck Surg 2012;138:301-306 


\section{Abstract}

\section{Objectives}

To assess visual acuity (VA) while the patient is walking and to evaluate oscillopsia severity in patients with bilateral vestibulopathy (BV) and in patients with unilateral vestibular loss (UVL).

\section{Design}

Prospective study with a group of patients with BV, a group of patients with UVL, and a control group of healthy subjects.

\section{Setting}

Tertiary academic center.

\section{Participants}

Thirty seven patients with BV(age range, 29-80 years), 11 patients with UVL (age range, 48-75 years), and 57 healthy subjects (age range 20-77 years).

\section{Intervention}

Computation of the difference between the VA measured in static conditions and in dynamic conditions while walking on a treadmill at 2, 4, and $6 \mathrm{~km} / \mathrm{h}$. Oscillopsia severity was assessed with a questionnaire that we developed.

\section{Main outcome measures}

Differences in VA at 2, 4, and $6 \mathrm{~km} / \mathrm{h}$ and oscillopsia severity score.

\section{Results}

As a group, patients with BV showed a significant increase of the VA differences compared with healthy subjects $(P<.001)$ and patients with UVL $(P<.001)$ for all 3 walking velocities. Normality thresholds were defined as healthy subjects' $95 \% \mathrm{Cl}$. Sensitivity of the test was $97 \%$ for discriminating patients with BV. Moderate to extreme oscillopsia severity was found in $81 \%$ of patients with BV and in $9 \%$ of patients with UVL. Differences in VA did not correlate with oscillopsia severity scores in patients with BV ( $P>.05$ for all comparisons).

\section{Conclusions}

We designed a highly sensitive, simple, cost-effective protocol to assess dynamic VA under physiologic conditions and a questionnaire to determine oscillopsia severity. Both tools could be used for the evaluation of new treatments for BV and patients with UVL. 


\section{Introduction}

Walking is probably the most common form of motion for an active adult. During moderately fast walking $(6 \mathrm{~km} / \mathrm{h})$, a combination of vertical and horizontal translational and rotational head movements is induced. Vertical head translation occurs at the stepping frequency $(2 \mathrm{~Hz})$, and lateral head translation at the stride frequency $(1 \mathrm{~Hz})$. Peak head pitch and yaw angular velocities are around $17^{\circ} / \mathrm{s}$, which is in the functional range of vestibuloocular reflex (VOR). ${ }^{1}$ The induced image slip on the retina is greatly compensated by the VOR, which generates compensatory eye movements opposite to head movements. In healthy subjects, a gain (eye velocity/head velocity) close to 1 allows efficient image stabilization on the retina. In with bilateral vestibulopathy (BV), the VOR is significantly impaired or absent. Thus, with high-frequency head movements $(>1 \mathrm{~Hz})$, the retinal slip cannot be optimally compensated. Because the threshold for perception of retinal drift is close to $1 \% \mathrm{~s}$ in healthy subjects, ${ }^{2}$ high-frequency head movements can lead to oscillopsia, an illusion of movement of the visual environment. For low-frequency head movements $(<1 \mathrm{~Hz})$, the optokinetic reflex and, to a certain extent, the cervicoocular reflex can generate compensatory eye movements. ${ }^{3}$ Efficient stabilization of the image on the retina is also necessary to preserve visual acuity (VA) during head movements. Indeed, VA decreases rapidly when the retinal slip exceeds the threshold of few degrees per second $\left(2.5^{\circ} / \mathrm{s}-4.0^{\circ} / \mathrm{s}\right) .^{4}$

Illusory perception of motion and decreased VA during head movements can be experienced simultaneously by patients with BV, and the combination is often described without distinction as "blurred vision," one of the main complaints of patients with BV, and can lead to a significant deterioration of quality of life. ${ }^{5}$ Although patients with BV have reported a certain subjective improvement after vestibular rehabilitation, ${ }^{6}$ there is unfortunately no evidence of an efficient treatment. Zingler et al. ${ }^{7}$ found that, independent of the etiology, in more than $80 \%$ of 82 patients with BV who had at least 3 months of vestibular training, there was neither a subjective improvement in their condition nor an improvement in their vestibular reflexes (follow-up range, 3 months to 13 years after initial diagnosis).

The idea of a vestibular implant that could provide the central nervous system with information about head angular velocities by electric stimulation of the vestibular nerve has emerged. Recently, partial recovery of the VOR has been achieved by electric stimulation of the semicircular canals of labyrinthine-defective monkeys. ${ }^{8-10}$ In humans, important steps toward the development of a vestibular implant have been made. Surgical approaches to electrode implantation sites have been described, ${ }^{11,12}$ and the first electric stimulations of the vestibular nerve have shown that it is possible to elicit controlled eye movements. ${ }^{13,14}$

If VOR can be restored in humans, specific tests to select and monitor vestibular implant candidates will be needed. Gaze stabilization can be tested with the Head 
Impulse Test $(\mathrm{HIT})^{15}$ in the 3 different semicircular ca- nals planes. The presence of corrective ocular saccades reveals an abnormal VOR function. ${ }^{15}$ Gaze stabilization performance can also be evaluated by measurement of the VA during active or passive, vertical and horizontal head movements; this process is called dynamic VA (DVA). ${ }^{16}$ Passive high angular velocity $\left(150^{\circ} / \mathrm{s}\right)$ movements allow best discrimination between patients with unilateral vestibular loss (UVL) or bilateral vestibular loss and normal subjects. ${ }^{17}$

A drawback of these approaches is the nonphysiologic stimuli that are applied. In the present study, we opted for VA measurements while the patient was walking on a treadmill at different velocities, which corresponds to physiologic stimuli. The fact that the test cannot discriminate the side, in a case of unilateral vestibular loss, is not relevant because we aim to select patients with bilateral impairment of the vestibular function.

Visual acuity testing has already been investigated under similar conditions, showing a significant DVA de- crease in patients with BV compared with healthy subjects. ${ }^{18,19}$ The correlation between DVA and oscillopsia severity was not assessed. For horizontal movements, it was shown that oscillopsia severity is most likely related to retinal slip tolerance level and not to retinal slip amplitude itself. ${ }^{20}$ Our aim was to develop and implement a DVA protocol for a patient walking on a treadmill that is simple, cost-effective, quick, and sensitive. Second we aimed to correlate DVA performance to oscillopsia severity assessed with a questionnaire that we also developed. Grading of oscillopsia severity has been previously demonstrated with a visual analog scale ${ }^{21}$ or a questionnaire developed by researchers for that specific purpose. ${ }^{20}$ Nevertheless, to our knowledge there is no standardized way of assessing oscillopsia severity. Both the DVA protocol and the oscillopsia severity questionnaire described herein should allow evaluation of treatments for patients with BV and patients with UVL.

\section{Methods}

\section{Test subjects}

Thirty-seven patients (20 men and 17 women; mean age, 56 years; age range, 29-80 years) with BV were observed at the Di- vision of Balance Disorders at Maastricht University Hospital between January 2010 and May 2011. They all fulfilled 3 inclusion criteria: (1) mean peak slow phase velocity of $5 \%$ s or less in bilateral bithermal caloric irrigations; (2) pathologic HIT for horizontal and vertical canals; and (3) a gain of $25 \%$ or less on rotatory chair tests. Electronystagmography (ENG) was used for vestibular testing. Bilateral bithermal $\left(30^{\circ} \mathrm{C}\right.$ and $\left.44^{\circ} \mathrm{C}\right)$ caloric irrigations with water were performed by experienced technicians under standard 
conditions. Rotatory chair tests consisted of horizontal torsion swing $(0.1 \mathrm{~Hz}$; maximum velocity, $100 \%$ s) and bilateral velocity steps (velocity, $250 \%$ s). Manual HITs were recorded with a high-speed camera (Casio Exilim, Pro EX-F1; Casio) at $12 \mathrm{X}$ optical zoom and 300 frames per second in the 3 semicircular canal planes. Presence of corrective saccades was considered pathologic. All recordings were analyzed by an experienced otorhinolaryngologist. All patients had a normal neurologic status and normal oculomotor function.

A group of 11 patients ( 7 men and 4 women; mean age, 61 years; age range, 48-75 years) with UVL was also included. They all fulfilled the following criteria: (1) unilateral mean peak slow phase velocity of $5 \%$ or less in bilateral bithermal caloric irrigations; (2) unilateral pathologic HIT for horizontal and vertical canals; and (3) a gain of more than $30 \%$ for horizontal torsion swing $(0.11 \mathrm{~Hz}$; maximum velocity, $100 \%$ s).

As a control group, 57 healthy subjects were included (32 men and 25 women; mean age, 41 years; age range, 20-77 of years), who had no prior vestibular symptoms and normal HIT findings in the 3 semicircular canal planes.

\section{Va testing}

Visual acuity was measured in the 3 groups using a chart of Sloan letters (CDHKNORSVZ). The chart consisted of rows of 5 randomly chosen letters. As for the standard Snellen chart used by ophthalmologists, VA decreased 0.1 log unit per row on the logMAR scale. The VA scale was adapted to the test subject distance, which was $2.8 \mathrm{~m}$ for all measurements. The chart was positioned at eye height. Visual acuity was tested binocularly. Measurements were performed with eye corrections in place for subjects who wore glasses or contact lenses. Measurement procedures were identical in static and dynamic conditions. The experimental setup was calibrated and validated by comparison with the known VA (determined at the department of ophthalmology) in 10 healthy subjects.

Starting with letters in a row corresponding to a VA value of 1.25 , test subjects had to read out the 5 letters from left to right. The VA value of the last row with at least 3 correctly named letters was recorded. Tests were performed while the subject was standing or walking on a standard fitness treadmill. The protocol started with evaluation of the static VA (SVA) while the subject was standing still on the treadmill. Then, VA was evaluated while the subject walked 2, 4, and $6 \mathrm{~km} / \mathrm{h}$. Static VA evaluation was repeated at the end of the procedure. For every measurement, the Sloan letter chart was replaced by a new one with a different letter order.

A safety string connected to the emergency treadmill brake was clipped to the test subject's waist. If the test subject felt that he or she could not walk at higher speed, the procedure was interrupted, and the last SVA measurement was recorded. All measurements were performed under controlled luminance. 


\section{Oscillopsia severity score}

A questionnaire assessing oscillopsia severity was developed specifically for this study and was administered to patients with BV and patients with UVL. Oscillopsia was defined as a "sensation that the visual environment is moving when it's not." The 9-item questionnaire investigated oscillopsia frequency in different situations encountered in daily life. Each item was scored 1 (never), 2 (seldom), 3 (sometimes), 4 (often), or 5 (always). Scores were averaged to provide an oscillopsia severity score ranging from 1 to 5 . A mean score higher than 3 was considered to indicate moderate to extreme oscillopsia severity. Internal consistency of the questionnaire was measured with the Cronbach $\alpha$ statistic. The coefficient a would be 1 if questionnaire items were perfectly correlated and 0 if they were completely independent. The Cronbach a was 0.88 in the group of patients with BV. Values above 0.7 are generally considered acceptable (Figure 3.1). ${ }^{22}$

1. Do you have the sensation that the visual environment is moving when it's not?

2. By dim light, do you have the sensation that what you see is not stable?

3. Is it difficult for you to recognize known faces when you are walking?

4. When you are reading, do you have the sensation that the text is not stable?

5. When you are watching television, do you have the sensation that the image is not stable?

6. When you are driving your car, do you have the sensation that what you see is not stable?

7. As a car passenger, do you have the sensation that what you see is not stable?

8. When you are riding bicycle, do you have the sensation that what you see is not stable?

9. When you are walking on uneven ground, do you have the sensation that what you see is not stable?

Figure 3.1 Oscillopsia severity questionnaire.

always $=5$, often $=4$, sometimes $=3$, seldom $=2$, never $=1$

\section{Data and statistical analysis}

For each velocity, the VA difference (mean SVA-DVA) was obtained. Five healthy subjects repeated the protocol 5 times. Measured VA differences were not significantly different (Friedman, Bonferroni corrected). The coefficient of variance was less than $20 \%$ for each velocity, indicating good test reproducibility. Linear regressions were used for evaluation of a correlation between healthy subjects' VA differences at 2, 4, and $6 \mathrm{~km} / \mathrm{h}$ and age. The VA differences in patients with BV were compared with those in healthy subjects and in patients with UVL using a nonparametric Mann-Whitney $U$ test. For all 3 velocities, patients with BV and patients with UVL were considered abnormal when the VA difference was above the threshold (healthy subjects' $95 \% \mathrm{Cl}$ [mean \pm 2 SDs]). Sensitivity of the protocol was determined for each velocity and for combinations of velocities with the following equation: true positives/(true positives + false negatives). True positives were patients with BV and an abnormal VA difference, and false negatives were patients with BV 
and a normal VA difference. For patients with BV and patients with UVL, correlations between the VA difference at each velocity and the oscillopsia severity score were determined with the Pearson correlation coefficient. Scores of each item of the oscillopsia severity questionnaire were compared between patients with patients with BV and those with UVL using a nonparametric Mann-Whitney $U$ test.

\section{Results}

In healthy subjects, regression analysis showed a significant relationship between VA differences and age at $4 \mathrm{~km} / \mathrm{h}(F=13.71, P<.001)$ and $6 \mathrm{~km} / \mathrm{h}(F=4.64, P=.04)$ but not at $2 \mathrm{~km} / \mathrm{h}(F=0.367, P=55)$. At $4 \mathrm{~km} / \mathrm{h}$, age accounted for $19 \%$ of the variance of the VA differences and at $6 \mathrm{~km} / \mathrm{h}, 6 \%$ (Figure 3.2). As a group, healthy subjects showed a significant increase of the mean VA difference between 4 and $6 \mathrm{~km} / \mathrm{h}(P=.002)$ but not between 2 and $4 \mathrm{~km} / \mathrm{h}(P=.06)$.

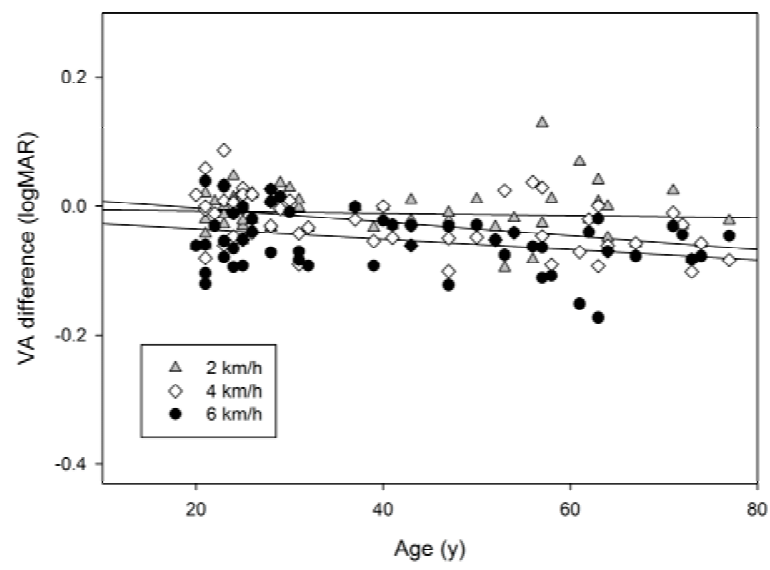

Figure 3.2 Visual acuity (VA) differences of 57 healthy subjects vs age at walking velocities of 2, 4 and $6 \mathrm{~km} / \mathrm{h}$. Regression analysis showed significant relationship between VA differences and age at 4 and $6 \mathrm{~km} / \mathrm{h}(P<.05$ for both) but not at $2 \mathrm{~km} / \mathrm{h}(P=.55)$.

Patients with BV were therefore compared with age- matched control group A, made up of 29 healthy subjects (16 men and 13 women; mean age, 56 years; age range, 32-77 years). Mean (SD) VA differences at 2,4 , and $6 \mathrm{~km} / \mathrm{h}$ were -0.166 (0.081), $-0.238(0.119)$, and $-0.340(0.138)$, respectively, in patients with BV and -0.015 (0.045), $-0.043(0.037)$, and $-0.066(0.041)$ in control group A. Mean VA differences were significantly higher in patients with BV than in healthy subjects at all 3 velocities $(P<.001$ for all). As a group, patients with BV showed a significant increase in their mean VA difference between 2 and $4 \mathrm{~km} / \mathrm{h}(P=.01)$ and between 4 and $6 \mathrm{~km} / \mathrm{h}(P=.03)$. 
Patients with UVL were compared with age-matched control group B, made up of 20 healthy subjects ( 11 men and 9 women; mean age, 60 years; age range 47-74 years). Mean (SD) VA differences at 2, 4, and $6 \mathrm{~km} / \mathrm{h}$ were $-0.022(0.032)$, $-0.061(0.047)$, and $-0.103(0.090)$, respectively, in patients with UVL and -0.011 $(0.052),-0.040(0.040)$, and $-0.069(0.045)$ in control group B. For all 3 velocities, the mean VA difference was not significantly different $(P>.05)$. As a group, patients with UVL showed a significant decrease in mean VA difference between 2 and $4 \mathrm{~km} / \mathrm{h}$ $(P=.03)$ but not between 4 and $6 \mathrm{~km} / \mathrm{h}(P=0.12)$.

Compared with patients with UVL, the mean VA difference in patients with BV was significantly increased at 2,4 , and $6 \mathrm{~km} / \mathrm{h}(P<.001$ for all) (Figure 3.3 ).

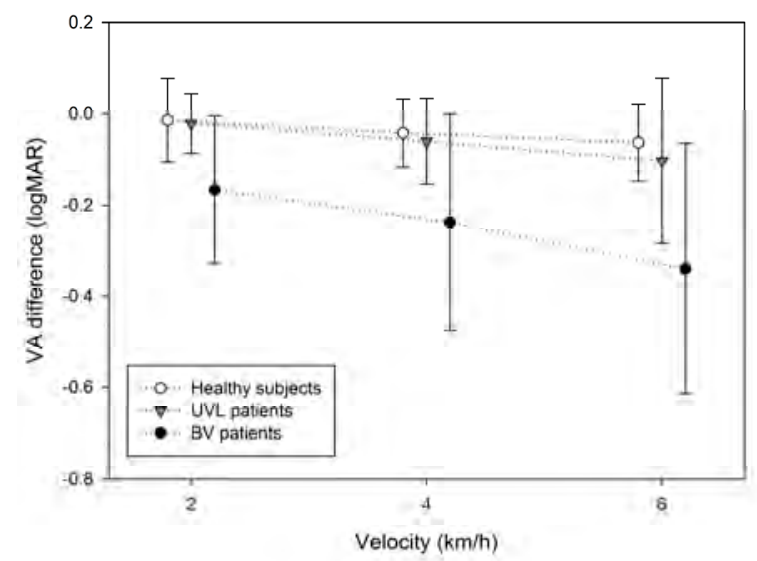

Figure 3.3 Mean visual acuity (VA) differences and 95\% Cls (mean $\pm 2 \mathrm{SDs}$ ). Illustrated are data for patients with bilateral vestibulopathy (BV), patients with unilateral vestibular loss (UVL), and age-matched control group A (29 healthy subjects) measured at walking velocities of 2, 4, and $6 \mathrm{~km} / \mathrm{h}$. The patients with BV differed significantly from both other groups at all velocities $(P<.001)$.

The mean VA difference $95 \% \mathrm{Cls}$ in control group A were $-0.091,-0.074$, and -0.147 at 2,4 , and $6 \mathrm{~km} / \mathrm{h}$, respectively. Those values were considered as normality thresholds. At $2 \mathrm{~km} / \mathrm{h}, 76 \%$ of patients with BV had an abnormal VA difference $(n=28)$. All patients with BV could perform the test at $2 \mathrm{~km} / \mathrm{h}$. At $4 \mathrm{~km} / \mathrm{h}, 84 \%$ of patients with BV had an abnormal VA difference $(n=31) ; 11 \%$ had a normal VA difference $(n=4)$ and $6 \%$ were not able to walk at that pace $(n=2)$. At $6 \mathrm{~km} / \mathrm{h}, 73 \%$ of patients with BV had an abnormal VA difference $(n=27) ; 6 \%$ had a normal VA difference $(n=2)$; and $22 \%$ could not walk so fast $(n=8)$.

Only 1 patient with BV had a normal VA difference at all 3 velocities. Two patients with BV had a normal VA difference at $4 \mathrm{~km} / \mathrm{h}$, with abnormal values at 2 and $6 \mathrm{~km} / \mathrm{h}$. One patient with BV had a normal VA difference at $6 \mathrm{~km} / \mathrm{h}$, with abnormal values at 2 and $4 \mathrm{~km} / \mathrm{h}$. 
The sensitivity of the complete protocol, including measurements of the VA difference at 2,4 , and $6 \mathrm{~km} / \mathrm{h}$, was $97 \%$. When testing patients' VA differences exclu- sively at $2 \mathrm{~km} / \mathrm{h}$ or $4 \mathrm{~km} / \mathrm{h}$, we found the sensitivities to be $76 \%$ and $84 \%$, respectively. By combining 2 and $4 \mathrm{~km} / \mathrm{h}$, we calculated the sensitivity to be $95 \%$.

The mean VA difference $95 \% \mathrm{Cls}$ in control group B were $-0.114,-0.120$, and -0.159 at 2, 4, and $6 \mathrm{~km} / \mathrm{h}$, respectively. Those values were considered normality thresholds. Among the patients with UVL, $100 \%$ had a normal VA difference at $2 \mathrm{~km} / \mathrm{h}(\mathrm{n}=11)$; $91 \%$ at $4 \mathrm{~km} / \mathrm{h}(\mathrm{n}=10)$; and $73 \%$ at $6 \mathrm{~km} / \mathrm{h}(\mathrm{n}=8)$. All of them could perform the test at all 3 velocities.

None of the healthy subjects complained of oscillopsia. In contrast, all patients with BV experienced oscillopsia. We collected 36 oscillopsia severity score questionnaires from patients with BV, and 11 from patients with UVL. Scores higher than 3 were considered to indicate moderate to extreme oscillopsia severity. No significant correlations were found at any of the 3 walking velocities between oscillopsia severity scores in patients with BV and VA differences ( $P>.05$ for all). In Figure 3.4, patients with BV and patients with UVL VA differences at $4 \mathrm{~km} / \mathrm{h}$ are plotted vs the oscillopsia severity scores. Of 35 patients with BV who could perform the test at $4 \mathrm{~km} / \mathrm{h}, 28$ had oscillopsia severity scores higher than 3 , indicating the presence of moderate to extreme oscillopsia severity. All but 1 patient with UVL had an oscillopsia severity score of 3 or lower. Among the 3 patients with UVL and a score of 3, 2 showed an abnormal VA difference at $6 \mathrm{~km} / \mathrm{h}$ (Figure 3.4).

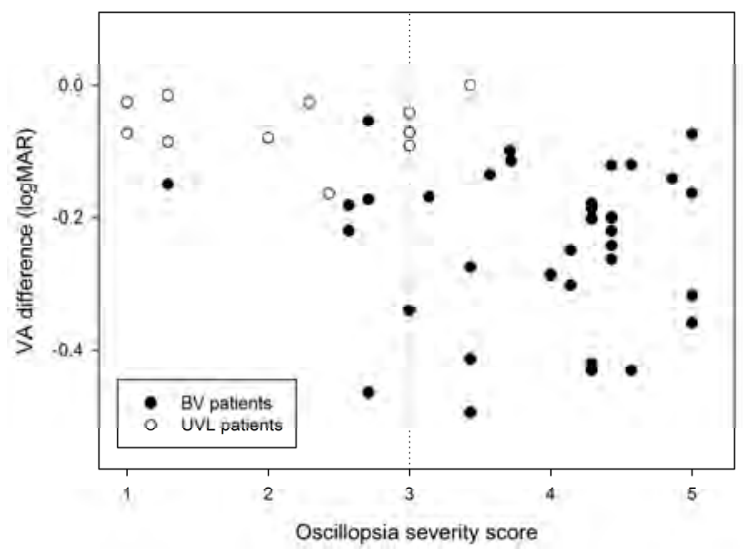

Figure 3.4 Visual acuity (VA) differences measured while subjects were walking at $4 \mathrm{~km} / \mathrm{h}$ vs oscillopsia severity scores. Illustrated are data from patients with bilateral vestibulopathy (BV) and patients with unilateral vestibular loss (UVL). No correlation was found ( $\mathrm{BV}=0.04$ $[P=.83] ; \mathrm{rUVL}=0.02[P=.96])$. Similar results were obtained at 2 and $6 \mathrm{~km} / \mathrm{h}$. The dotted vertical line indicates an oscillopsia severity score of 3; a score higher than 3 indicated moderate to extreme oscillopsia severity. 
Except for the mean score of item 5, patients with BV, as a group, had significantly higher individual item scores than patients with UVL $(P<.05$ for all comparisons) (Figure 3.5).

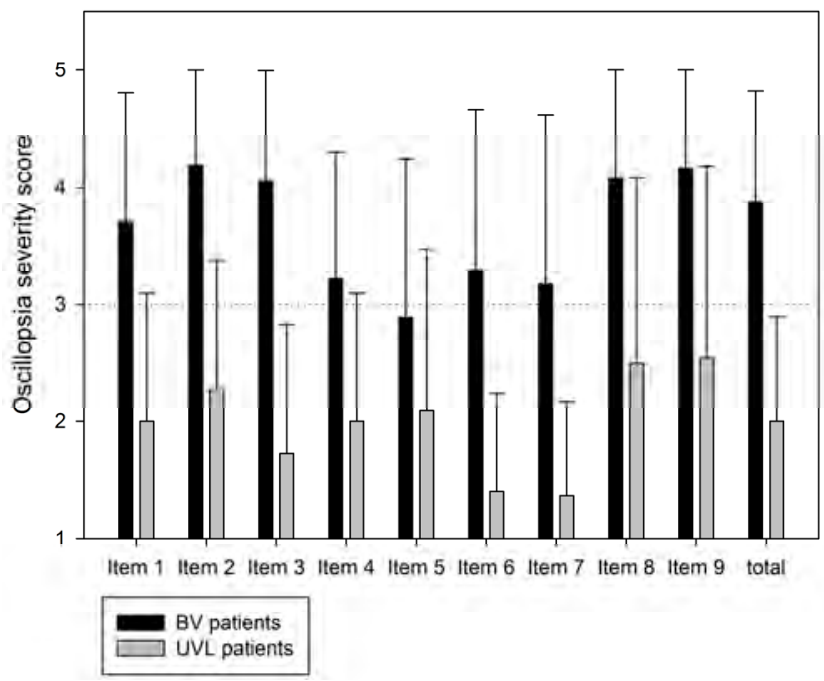

Figure 3.5 Oscillopsia severity questionnaire scores. Illustrated are mean (SD) individual item scores and mean (SD) total oscillopsia severity scores for the group of patients with bilateral vestibulopathy (BV) and patients with unilateral vestibular loss (UVL). Scores higher than 3 (indicated by the horizontal dotted line) indicated moderate to extreme oscillopsia severity. Except for the score of item 5, all scores were significantly higher in the group of patients with $\mathrm{BV}(\mathrm{P}<.05$ for all but item 5$)$.

\section{Comment}

To our knowledge, the present study includes the larg- est group of patients with BV ever evaluated for DVA and oscillopsia severity. The proposed protocol for DVA evaluation has a high sensitivity for discriminating BV. Our data would suggest that VA measurements should be per-formed while the patient is walking at 2 and $4 \mathrm{~km} / \mathrm{h}$. In cases of normal VA differences, testing at $6 \mathrm{~km} / \mathrm{h}$ should be added. It is a simple, costeffective procedure that can be performed in less than 10 minutes. Prior practice or training is not necessary to perform the test, which can easily be performed by a single person. Most patients with BV could perform the test without difficulties. Nevertheless, owing to imbalance, 2 of them could not walk at $4 \mathrm{~km} / \mathrm{h}$, and 8 could not walk at $6 \mathrm{~km} / \mathrm{h}$.

This protocol evaluates the vestibular system in physiologic conditions and could be used as a complementary vestibular clinical test. Moreover, in contrast to other 
commonly used vestibular tests such as caloric or rotatory tests, specific training in neurotology is not necessary for adequate interpretation of the results. This test could therefore be used by general practitioners, neurologists, or otorhinolaryngologists to reveal BV in patients presenting with oscillopsia. More specifically, it could help assess the functional outcome of a vestibular training protocol. Regarding the development of a vestibular implant, we think that our DVA protocol is suitable for candidate selection and might also play a role in assessment of the functional benefit after implantation.

Among patients with BV, walking at a velocity as low as $2 \mathrm{~km} / \mathrm{h}$ already induced a substantial VA decrease. The severity of oscillopsia was not correlated with the amount of VA loss during walking at any of the 3 tested velocities. This is in accordance with findings of a previous study ${ }^{21}$ using a DVA protocol with predictable active head movements. Nevertheless, this study was limited to a small number of patients with BV $(n=13)$, and only the DVA improvement after vestibular training was considered, not the absolute value of the DVA. ${ }^{21}$

A DVA protocol with passive head movements should be preferred because patients with BV can develop central mechanisms that could partially substitute for the VOR, such as compensatory corrective saccades, which are more effective during selfgenerated than manually imposed head rotations. ${ }^{16}$ It has been shown, using a selfmotion vs. a visual motion psychophysical test and a self-perceived handicap questionnaire, that amplitude of retinal slip was not correlated with the severity of the handicap due to oscillopsia. ${ }^{20}$ By increasing their threshold for visual motion detection as a result of a central adaptive process, patients with BV could improve their tolerance to retinal slip. ${ }^{23,24}$ On the other hand, in a functional MRI study, it was shown that when patients with BV were compared with healthy subjects, optokinetic stimulation induced a higher activation of the visual cortex and oculomotor areas in patients with BV, especially of the primary visual cortex and of the motion-sensitive areas in the temporal lobe. ${ }^{25}$ It is still not clear how these 2 apparently opposed mechanisms interact during locomotion, for instance.

In the present study, none of the healthy subjects reported oscillopsia. As a group, our patients with BV scored significantly higher than patients with UVL for 8 of the 9 oscillopsia severity questionnaire items. Items 4 and 5 revealed that patients with BV can experience oscillopsia while reading or watching television. This finding indicates that certain situations that are considered static for healthy subjects should be considered dynamic for patients with BV. Indeed, most patients with BV mentioned the necessity for active head stabilization in such situations. Only 1 of our patients with UVL had an oscillopsia severity score higher than 3 (9\%), whereas 29 patients with BV had a score higher than $3(81 \%)$. This finding indicates clearly that despite having central adaptive mechanisms increasing their tolerance to retinal slip, ${ }^{20}$ most patients with BV experience moderate to extreme oscillopsia severity. These results seem to indicate that unilateral recovery of the VOR might reduce significantly the 
severity of oscillopsia. Furthermore, the oscillopsia severity score could be used to assess the subjective benefit of treatments, such as a vestibular training protocol, in patients with BV and UVL.

Our results showed a significant relationship between VA differences and age when walking at 4 and $6 \mathrm{~km} / \mathrm{h}$. Age accounted for $19 \%$ of the variations of the VA differences at $4 \mathrm{~km} / \mathrm{h}$ and for $6 \%$ at $6 \mathrm{~km} / \mathrm{h}$. This is slightly higher than reported in a study using a DVA protocol with high angular velocity $\left(>150^{\circ} / \mathrm{s}\right)$ elicited by passive head movements around the yaw axis, where age accounted only for $4 \%$ of the VA variations in 100 healthy subjects. ${ }^{17}$ In another study, using a DVA protocol with predictable active head movements, age accounted for $40 \%$ of the DVA variations in healthy subjects. ${ }^{26}$

Among our healthy subjects and patients with BV, only 1 was older than 75 years. For this population, VOR performances are known to be significantly diminished. ${ }^{27}$

Therefore, a significant DVA performance decrease can be expected, which could also explain abnormal VA differences at $6 \mathrm{~km} / \mathrm{h}$ in 2 patients with UVL who were older than 75 years. Apart from those 2 patients, the only abnormal VA difference among patients with UVL was found in a 64-year-old patient at $6 \mathrm{~km} / \mathrm{h}$, which indicates that in most cases, unilateral recovery of the VOR could potentially allow normalization of the DVA when walking.

In conclusion, the DVA protocol proposed in this study is simple, quick, and costeffective. It can reveal a bilateral vestibulopathy with a sensitivity as high as $97 \%$. The oscillopsia severity scores showed that despite central adaptive mechanisms, most patients with BV experience moderate to extreme oscillopsia severity. There is no clear correlation between the DVA and oscillopsia severity. Both the DVA protocol and the oscillopsia severity score could be used to assess the functional and subjective benefits of treatments, such as vestibular training, for patients with BV and UVL. Furthermore, it could help to select and monitor potential vestibular implant candidates. 


\section{References}

1. Moore ST, Hirasaki E, Raphan T, Cohen B. The human vestibulo-ocular reflex during linear locomotion. Ann N Y Acad Sci 2001;942:139-47.

2. Murakami I. Correlations between fixation stability and visual motion sensitivity. Vision Res 2004;44: 751-61.

3. Barnes GR, Forbat LN. Cervical and vestibular afferent control of oculomotor response in man. Acta Otolaryngol 1979;88:79-87.

4. Leigh RJ, Averbuch-Heller L, Tomsak RL, Remler BF, Yaniglos SS, Dell'Osso LF. Treatment of abnormal eye movements that impair vision: strategies based on current concepts of physiology and pharmacology. Ann Neurol 1994;36:129-41.

5. Guinand N, Boselie F, Guyot JP, Kingma H. Abstracts of the XXVI Ba'ra'ny Society Meeting, Reykjavik, Iceland, August 18-21, 2010. J Vestib Res 2010;20:79-340.

6. Gillespie MB, Minor LB. Prognosis in bilateral vestibular hypofunction. Laryngoscope 1999;109:35-41.

7. Zingler VC, Weintz E, Jahn K, et al. Follow-up of vestibular function in bilateral vestibulopathy. J Neurol Neurosurg Psychiatry 2008;79(3):284-8.

8. Dai C, Fridman GY, Davidovics NS, Chiang B, Ahn JH, Della Santina CC. Restoration of 3D vestibular sensation in rhesus monkeys using a multichannel vestibular prosthesis. Hear Res 2011;281):74-83.

9. Dai C, Fridman GY, Della Santina CC. Effects of vestibular prosthesis electrode implantation and stimulation on hearing in rhesus monkeys. Hear Res 2011;277:204-10.

10. Merfeld DM, Haburcakova C, Gong W, Lewis RF. Chronic vestibulo-ocular reflexes evoked by a vestibular prosthesis. IEEE Trans Biomed Eng 2007;54:1005-5.

11. Kos MI, Feigl G, Anderhuber F, Wall C, Fasel JH, Guyot JP. Transcanal approach to the singular nerve. Otol Neurotol 2006;27(4):542-6.

12. Feigl GC, Fasel JH, Anderhuber F, et al. Superior vestibular neurectomy: a novel transmeatal approach for a denervation of the superior and lateral semicircular canals. Otol Neurotol 2009;30:586-91.

13. Wall C III, Kos MI, Guyot JP. Eye movements in response to electric stimulation of the human posterior ampullary nerve. Ann Otol Rhinol Laryngol 2007;116:369-74.

14. Guyot JP, Sigrist A, Pelizzone M, Kos MI. Adaptation to steady-state electrical stimulation of the vestibular system in humans. Ann Otol Rhinol Laryngol 2011;120:143-9.

15. Halmagyi GM, Curthoys IS. A clinical sign of canal paresis. Arch Neurol 1988;45:737-9.

16. Tian JR, Shubayev I, Demer JL. Dynamic visual acuity during passive and self-generated transient head rotation in normal and unilaterally vestibulopathic humans. Exp Brain Res 2002;142:486-95.

17. Vital D, Hegemann SC, Straumann D, et al. A new dynamic visual acuity test to assess peripheral vestibular function. Arch Otolaryngol Head Neck Surg. 2010;136:686-91.

18. Lambert S, Sigrist A, Delaspre O, Pelizzone M, Guyot JP. Measurement of dynamic visual acuity in patients with vestibular areflexia. Acta Otolaryngol 2010;130:820-3.

19. Hillman EJ, Bloomberg JJ, McDonald PV, Cohen HS. Dynamic visual acuity while walking in normals and labyrinthine-deficient patients. J Vestib Res 1999;9:49-57.

20. Grunfeld EA, Morland AB, Bronstein AM, Gresty MA. Adaptation to oscillopsia: a psychophysical and questionnaire investigation. Brain 2000;123:277-90.

21. Herdman SJ, Hall CD, Schubert MC, Das VE, Tusa RJ. Recovery of dynamic visual acuity in bilateral vestibular hypofunction. Arch Otolaryngol Head Neck Surg 2007;133:383-9.

22. Nunnally JC. Psychometric Theory. 2nd ed. New York, NY: McGraw-Hill; 1978.

23. Morland $\mathrm{AB}$, Bronstein $\mathrm{AM}$, Ruddock $\mathrm{KH}$, Wooding DS. Oscillopsia: visual function during motion in the absence of vestibulo-ocular reflex. J Neurol Neurosurg Psychiatry 1998;65:828-35.

24. Kalla R, Muggleton N, Spiegel R, et al. Adaptive motion processing in bilateral vestibular failure. J Neurol Neurosurg Psychiatry 2011;82:1212-6.

25. Dieterich M, Bauermann T, Best C, Stoeter P, Schlindwein P. Evidence for cortical visual substitution of chronic bilateral vestibular failure (an fMRI study). Brain 2007;130:2108-16.

26. Herdman SJ, Schubert MC, Tusa RJ. Role of central preprogramming in dynamic visual acuity with vestibular loss. Arch Otolaryngol Head Neck Surg 2001;127:1205-10. 
Chapter 3

27. Baloh RW, Jacobson KM, Socotch TM. The effect of aging on visual-vestibuloocular responses. Exp Brain Res 1993;95:509-16. 


\section{Chapter 4}

Vestibular implants: 8 years of experience with
electrical stimulation of the vestibular nerve in 11
patients with bilateral vestibular loss

N Guinand, R van de Berg, S Cavuscens, RJ Stokroos, M Ranieri, M Pelizzone, H Kingma, J-P Guyot, A Perez-Fornos

ORL J Otorhinolaryngol Relat Spec 2015;77(4):227-240 


\section{Abstract}

\section{Background}

The concept of the vestibular implant is primarily to artificially restore the vestibular function in patients with a bilateral vestibular loss (BVL) by providing the central nervous system with motion information using electrical stimulation of the vestibular nerve. Our group initiated human trials about 10 years ago.

\section{Methods}

Between 2007 and 2013, 11 patients with a BVL received a vestibular implant prototype providing electrodes to stimulate the ampullary branches of the vestibular nerve. Eye movements were recorded and analyzed to assess the effects of the electrical stimulation. Perception induced by electrical stimulation was documented.

\section{Results}

Smooth, controlled eye movements were obtained in all patients showing that electrical stimulation successfully activated the vestibulo-ocular pathway. However, both the electrical dynamic range and the amplitude of the eye movements were variable from patient to patient. The axis of the response was consistent with the stimulated nerve branch in 17 out of the 24 tested electrodes. Furthermore, in at least 1 case, the elicited eye movements showed characteristics similar to those of compensatory eye movements observed during natural activities such as walking. Finally, diverse percepts were reported upon electrical stimulation (i.e., rotatory sensations, sound, tickling or pressure) with intensity increasing as the stimulation current increased.

\section{Conclusion}

These results demonstrate that electrical stimulation is a safe and effective means to activate the vestibular system, even in a heterogeneous patient population with very different etiologies and disease durations. Successful tuning of this information could turn this vestibular implant prototype into a successful artificial balance organ. 


\section{Introduction}

Bilateral loss of the vestibular function (BVL) has a dramatic impact on the quality of life. The affected patients complain predominantly of chronic imbalance and blurred vision in dynamic conditions. ${ }^{1.2}$ They fear to fall and frequently report having difficulties reading signs or recognizing faces when they are walking. Some even report feeling ashamed to be seen in public as others often think they are drunk. Moreover, due to multifocal cortical and thalamic projections of vestibular afferents, emotions, memory, cognitive abilities and personality can also be affected..$^{3-5}$ In most cases, a BVL cannot be compensated and sensory substitution is insufficient so that there is no or little spontaneous improvement to be expected in the long term. ${ }^{6}$ As a consequence, BVL imposes a significant social and economic burden on patients and society. ${ }^{7}$ Unfortunately, there currently is no evidence for an efficient treatment.

The concept of a vestibular implant to artificially restore the vestibular function is similar to that of cochlear implants, which have a proven track record for hearing rehabilitation in cases of profound deafness. Briefly, in a vestibular implant, head motion is captured with motion sensors (i.e., gyroscopes) and transformed to a pattern of electrical currents by an external processor. This information is then wirelessly transmitted to an implanted stimulator that incorporates vestibular electrodes. Electrical stimulation delivered through these vestibular electrodes would trigger action potentials in the vestibular nerve that, in theory, would be interpreted by the central nervous system as head motion, ultimately allowing the 'artificial' restoration of the vestibular function.

Intensive efforts towards the development of a vestibular implant for clinical applications have been undertaken during the past decade. ${ }^{8}$ Devices with single or multichannel independent vestibular arrays have been designed and/or manufactured. ${ }^{9-13}$ Different motion sensor fixation and signal processing strategies have been proposed, leading to the recent filing of several patents. ${ }^{14-18}$ Finally, several animal and human studies have established the feasibility of this concept. Electrical stimulation was identified as an effective means for activating the vestibular system in animals already in the 1960s. ${ }^{19.20}$ Most recent animal research efforts have concentrated on meticulously investigating the effects of electrical stimulation parameters on vestibular responses, ${ }^{21}$ focusing mainly on vestibulo-ocular responses, $^{22-34}$ but also on orientation percepts and postural responses. ${ }^{35-37}$ In humans, extralabyrinthine and intralabyrinthine surgical routes to the lateral (LAN), posterior (PAN) and superior (SAN) ampullary branches of the vestibular nerve have been described and validated in peroperative stimulation trials. ${ }^{38-43}$ Our group has reported on the results of the first chronic implantations of a vestibular implant prototype in human subjects, ${ }^{44}$ followed by a group at the University of Washington. ${ }^{45}$ Our most recent results demonstrated that it is possible to elicit an artificial, motion- 
controlled vestibulo-ocular reflex in implanted patients. ${ }^{46}$ Postural responses to electrical stimulation have also been reported in human subjects. ${ }^{47}$

Our research group has participated in vestibular implant development for over 10 years. Today, we have a unique pool of 11 BVL patients implanted with a vestibular implant prototype. In this paper, we report on our main results, gathered over a period of 8 years.

\section{Materials and methods}

\section{Patients and surgery}

Eleven patients with bilateral or unilateral deafness ( 3 females and 8 males, mean age at implantation years, range 34-71 years) and concomitant BVL were recruited between 2007 and 2013 at the Service of Otorhinolaryngology, Head and Neck Surgery, Department of Clinical Neurosciences, Geneva University Hospital in Switzerland and at the Division of Balance Disorders of Maastricht University Medical Centre in the Netherlands. The demographics of the patient population are presented in Table 4.1. They all fulfilled 3 inclusion criteria: (1) mean peak slow-phase velocity of $\leq 5 \% \mathrm{~s}$ in bilateral bithermal caloric irrigations, (2) pathological Head Impulse Test for all 6 horizontal and vertical canals, and $(3)$ low $(<0.2)$ or no gain in rotatory chair tests. Standard videonystagmography and electronystagmography were used for vestibular testing. Bilateral bithermal $\left(30\right.$ and $44^{\circ} \mathrm{C}$ ) caloric irrigations were performed by highly experienced technicians in standardized conditions. Rotatory chair tests consisted of standard clinical horizontal torsion swing tests $\left(0.05-0.1 \mathrm{~Hz}, \omega \max =60^{\circ} / \mathrm{s}\right)$. The Head Impulse Test was performed with the Video Head Impulse Test of Ulmer (Synapsis@, Marseille, France), the EyeSeeCam Video Head Impulse Test (EyeSeeCam VOG @, Munich, Germany) and/or the ICS Impulse (Otometrics, Denmark).

The patients received a custom-modified cochlear implant (MED-EL, Innsbruck, Austria) with 1, 2 or 3 vestibular electrodes derived from the main cochlear array (Figure 4.1). A minimum of 9 electrodes was thus left for the cochlear stimulation, which should not jeopardize the auditory outcome. ${ }^{48}$ 
Table 4.1 Demographics and implant details for each patient.

\begin{tabular}{|c|c|c|c|c|c|c|c|c|c|}
\hline Patient & Sex & $\begin{array}{l}\text { Hearing } \\
\text { loss }\end{array}$ & Etiology & Onset & $\begin{array}{l}\text { Age at } \\
\text { implant }\end{array}$ & $\begin{array}{l}\text { Implant } \\
\text { year }\end{array}$ & $\begin{array}{l}\text { Implanted } \\
\text { side }\end{array}$ & $\begin{array}{l}\text { Vestibular } \\
\text { electrodes }\end{array}$ & $\begin{array}{l}\text { Surgical } \\
\text { approach }\end{array}$ \\
\hline S1 & $M$ & $B$ & idiopathic & progressive & 68 & 2007 & left & PAN & $E L$ \\
\hline S2 & M & B & $\begin{array}{l}\text { congenital / } \\
\text { idopathic }\end{array}$ & progressive & 34 & 2008 & right & PAN & EL \\
\hline S3 & $M$ & B & $\begin{array}{l}\text { congenital / } \\
\text { idopathic }\end{array}$ & progressive & 46 & 2008 & left & PAN & EL \\
\hline S4 & $M$ & $B$ & Menière's & progressive & 71 & 2011 & left & PAN & EL \\
\hline S5 & M & $\mathrm{U}$ & traumatic & $\begin{array}{c}\text { acute } \\
\text { (<1 year } \\
\text { ago) }\end{array}$ & 63 & 2012 & right & $\begin{array}{l}\text { PAN } \\
\text { LAN }\end{array}$ & EL \\
\hline S6 & $\mathrm{F}$ & B & $\begin{array}{c}\text { mastoidectomy }(\mathrm{L}) \\
\text { traumatic }(\mathrm{R})\end{array}$ & $\begin{array}{l}\text { acute } \\
\text { (<1 year } \\
\text { ago) }\end{array}$ & 67 & 2013 & left & $\begin{array}{l}\text { PAN } \\
\text { LAN } \\
\text { SAN }\end{array}$ & IL \\
\hline S7 & $\mathrm{F}$ & $U$ & meningitis & $\begin{array}{c}\text { acute } \\
\text { (47 years } \\
\text { ago) }\end{array}$ & 48 & 2012 & right & $\begin{array}{l}\text { PAN } \\
\text { LAN } \\
\text { SAN }\end{array}$ & IL \\
\hline S8 & M & B & DFNA9 & progressive & 67 & 2012 & left & $\begin{array}{l}\text { PAN } \\
\text { LAN } \\
\text { SAN }\end{array}$ & IL \\
\hline S9 & $\mathrm{F}$ & B & DFNA9 & progressive & 68 & 2013 & left & $\begin{array}{l}\text { PAN } \\
\text { LAN } \\
\text { SAN }\end{array}$ & IL \\
\hline S10 & $M$ & B & DFNA9 & progressive & 66 & 2013 & left & $\begin{array}{l}\text { PAN } \\
\text { LAN } \\
\text { SAN }\end{array}$ & IL \\
\hline S11 & $M$ & B & DFNA9 & progressive & 64 & 2013 & left & $\begin{array}{l}\text { PAN } \\
\text { LAN } \\
\text { SAN }\end{array}$ & IL \\
\hline
\end{tabular}

$\mathrm{B}=$ bilateral; $\mathrm{U}=$ unilateral; $\mathrm{EL}$ = extralabyrinthine; $\mathrm{IL}=$ intralabyrinthine

In 5 patients (S1-S5), an extralabyrinthine transmeatal surgical approach was performed. ${ }^{38,41}$ The PAN (S1-S5) and the LAN (only S5) were exposed. In these patients (except S1), this part of the surgery was done under local anesthesia, and a probe electrode (125 $\mu \mathrm{m}$ diameter, 90\% platinium-10\% iridium Tefloncoated wire; MicroProbes for Life Science, Gaithersburg, Md., USA) was used to perform acute peroperative electrical stimulation. The depth and direction of drilling was adjusted following the observation of nystagmic responses obtained upon electrical stimulation. Once the optimum electrode position was found, general anesthesia was induced and the custom-modified device was implanted using a conventional retroauricular approach with a regular mastoidectomy, a posterior tympanotomy and a cochleostomy. The cochlear array was inserted into the cochlea and the vestibular electrodes were put in contact with the PAN (and LAN in S5) and secured with fascia from the temporal muscle and/or glass ionomer (Ketac, 3M, Saint Louis, Minn., USA). 
(A)

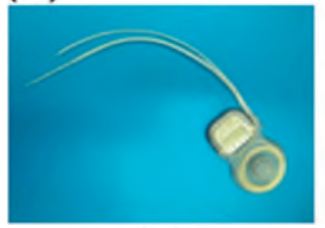

(C)
(B)

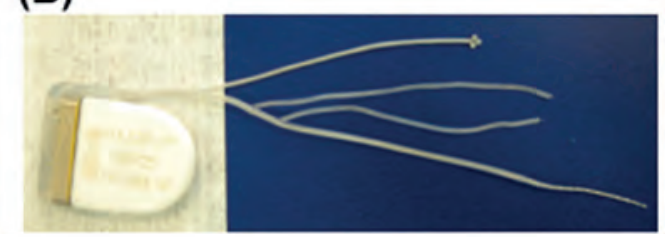

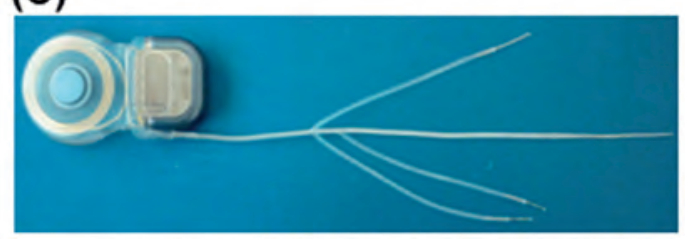

Figure 4.1 The three generations of vestibular implant prototypes developed in collaboration with MEDEL (Innsbruck, Austria) based on existing cochlear implant technology. A standard cochlear implant was customized by removing 1-3 electrodes from the cochlear array. Each of these 'vestibular' electrodes was located on the distal tip of separate leads to allow implantation in the posterior ampullary nerve (extralabyrinthine approach, a), in the posterior and lateral ampullary nerves (extralabyrinthine approach, b), or in the ampullae of the posterior, lateral, and superior semicircular canals (intralabyrinthine approach, c).

In 6 patients (S6-S11), an intralabyrinthine approach was used to put 1 electrode in each ampulla. ${ }^{43}$ The entire procedure was performed under general anesthesia. Briefly, this approach consisted of a regular mastoidectomy, a posterior tympanotomy and a cochleostomy. The 3 semicircular canals were blue-lined and an inframillimetric 'canalotomy' was performed close to each ampulla. Electrodes were then inserted into each ampulla. At this stage, the patient was kept under intravenous remifentanil only, allowing to maintain general anesthesia while preserving the slow phase of the vestibulo-ocular reflex (e.g., tonic eye deviation). Peroperative electrical stimulation delivered via the vestibular electrodes was performed and the direction of the resulting tonic eye deviation was used to adjust the electrode position. Finally, electrodes were secured with hydroxylapatite bone cement (Otomimix, Walter Lorenz Surgical, Jacksonville, Fla., USA) and fibrin sealant (Tissucol, Baxter International Inc., Deerfield, III., USA), or with fascia from the temporal muscle.

Device activation took place no earlier than 4 weeks postoperatively, when healing of the surgery site was assumed to be complete. For simplicity purposes, from now on, we will refer to PAN, LAN and SAN for electrical stimulation delivered with each of the vestibular electrodes. 


\section{Electrical stimulation}

The setup for the electrical stimulation of the PAN, LAN and SAN was composed of a desktop computer that allows customization of the stimulation parameters (current intensity, pulse rate, phase width, modulation depth and modulation frequency). The computer communicated this information to the implanted stimulator via the manufacturer's research interface Board (RIB II, MED-EL) and the system's antenna.

Cochlear electrodes were always switched off during the experimental procedure. Stimulation was delivered to each electrode separately, and consisted of trains of charge-balanced, cathodic-first, biphasic pulses (400 $\mu \mathrm{s} /$ phase) presented at 200 pulses/s. During the device activation, the current amplitude was incremented by steps of a maximum of $50 \mu \mathrm{A}$ (lowered to $25-10 \mu \mathrm{A}$ if necessary) to minimize patient discomfort. Vestibular threshold was determined as the first (lowest) level of electrical current where the first vestibular symptom was observed (e.g., a change in nystagmus slow-phase velocity $>2 \%$ ) or reported (e.g., 'I feel like turning'). Particular attention was given to the first reported perception. Then stimulation was again increased by 10- to $25-\mu \mathrm{A}$ steps until the upper comfortable level (e.g., occurrence of pain or facial nerve stimulation) was reached. The dynamic range was determined as the current range from the vestibular threshold up to the upper comfortable level. ${ }^{44}$

The next step consisted of characterizing the eye movements that could be elicited by electrical stimulation delivered through each electrode (single-electrode stimulation). A 'baseline' stimulation (constant amplitude electrical stimulation) was given at an amplitude arbitrarily chosen in the middle of the dynamic range. Once patients were adapted to this 'baseline' stimulation, ${ }^{44}$ the amplitude of the stimulus was modulated using a sinusoidal signal with a frequency of $3 \mathrm{~Hz}$ and a modulation depth corresponding to $75 \%$ of each patient's dynamic range. At the end of the experiments, 'baseline' stimulation was gradually decreased to zero.

\section{Eye movement recording and analysis}

Two-dimensional eye-in-head angular position was recorded using a fast monocular 2D video oculography system (EyeSeeCam VOG). Ideally, 3D binocular movements should be reported. ${ }^{49}$ However, we decided to use 2D video oculography for several reasons. First, although the search coil technique is considered as the gold standard for $3 D$ ocular recording, ${ }^{50}$ it is invasive and is generally not well tolerated for use longer than 20-30 min; therefore, we did not consider it acceptable to add this burden to our test patients who had to undergo long, repeated testing sessions. Secondly, search coil measurements require a relative complex infrastructure consisting of a cubic structure (about $1 \mathrm{~m}^{3}$ ) incorporating the 3D coils by which the magnetic field is generated. For accurate measurements, the patient's head must stay in the linear area (center) of this magnetic field. This is a major drawback as our future goal is to test patients at least partly moving freely in their environment. Moreover, 
previous studies have demonstrated that 2D analysis of eye movements is sufficient to assess the vestibulo-ocular reflex during natural activities. ${ }^{51}$ Thirdly, several portable, light-weight, high-speed, infrared monocular video oculography systems, incorporating motion sensors have recently been developed, providing accurate measurements of 2D eye movements and $6 \mathrm{D}$ head movement, ${ }^{52,53}$ but the detection of the eye torsion (3rd dimension) by video eye trackers is still more troublesome, less reliable and less accurate. Therefore, being aware of its limitations, we considered it acceptable to use monocular 2D video oculography as a first approach. All eye movement recordings were done in darkness with patients sitting in an upright position.

A segment of 10 cycles was analyzed for each experimental trial. Eye position data were first filtered at $30 \mathrm{~Hz}$ with a low-pass moving average filter (zero-phase shift). Eye velocity and acceleration were then obtained via the first and second derivatives of the eye position. Blinks and quick eye movements (e.g., saccades and nystagmus quick phases) were detected as segments where eye acceleration was $>1,000^{\circ} / \mathrm{s}$. These segments were removed from the data and were not replaced by interpolated values (Figure 4.2a).

Peak horizontal and vertical velocity (respectively, PVhorizontal and PVvertical) were estimated using best-fit frequency-fixed sinusoids (Figure 4.2b). Total peak velocity was then computed as the vector norm of these 2D components:

$$
\left(\sqrt{P V_{\text {horizontal }^{2}}+P V_{\text {vertical }}^{2}}\right) .
$$

The axis of eye movements (angle with respect to the horizontal) was computed as:

$$
\tan ^{-1}\left(\frac{P V_{\text {vertical }}}{P V_{\text {horizontal }}}\right)
$$

Eye movements with an angle $>45^{\circ}$ were considered as predominantly vertical and those with an angle $<45^{\circ}$ as predominantly horizontal. Finally, asymmetry was presented using the index

$$
\frac{P V_{E}-P V_{1}}{P V_{E}+P V_{1}},
$$

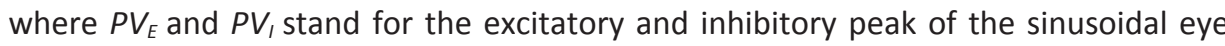
movement, respectively (i.e., away from and towards the implanted ear). These excitatory/inhibitory peak velocities were calculated using best fits to stimulus halfcycles (e.g., only positive or only negative), similar to previous studies. ${ }^{31,46}$ Individual patient results did not always follow a normal distribution; therefore, results were reported as median values (25th-75th percentiles). Mean results across patients 
followed a normal distribution (Shapiro-Wilk $P>0.05$ ) and were therefore presented as mean values ( \pm standard deviation).

\section{Ethics considerations}

Experiments were designed and conducted in accordance with the 1964 Declaration of Helsinki. Local Ethics Committees of the Geneva University Hospitals (NAC 11-080) and of the Maastricht University Medical Centre (NL36777.068.11/METC 11-2-031) approved this experimental protocol. All participants gave their informed consent prior their inclusion in the study.

\section{Results}

A total of 24 vestibular electrodes were available for electrical stimulation in 11 patients. At the time of writing this paper, the longest follow-up period was 8 years (patient S1, implantation in July 2007), and the shortest was 2 years (S10 and S11, implantation in July 2013). No complications related to the surgery or to the experimental procedure were reported.

\section{Measured dynamic range}

A vestibular threshold could be determined in 21 of the 24 available electrodes (blue circles in Figure 4.3). In 19 of these electrodes, the upper comfortable level corresponded to facial nerve stimulation (red circles in Figure 4.3). In 2 electrodes, no upper comfortable level could be determined even at the highest current amplitude tested $(550 \mu \mathrm{A}$; red triangles in Figure 4.3$)$. The dynamic range was highly variable across patients and across electrodes (gray columns in Figure 4.3). It was null for 1 electrode. In 3 electrodes, no vestibular reaction was observed nor reported, even at the highest stimulation currents tested $(550 \mu \mathrm{A}$; blue triangles in Figure 4.3$)$. 
(A)

Raw position data
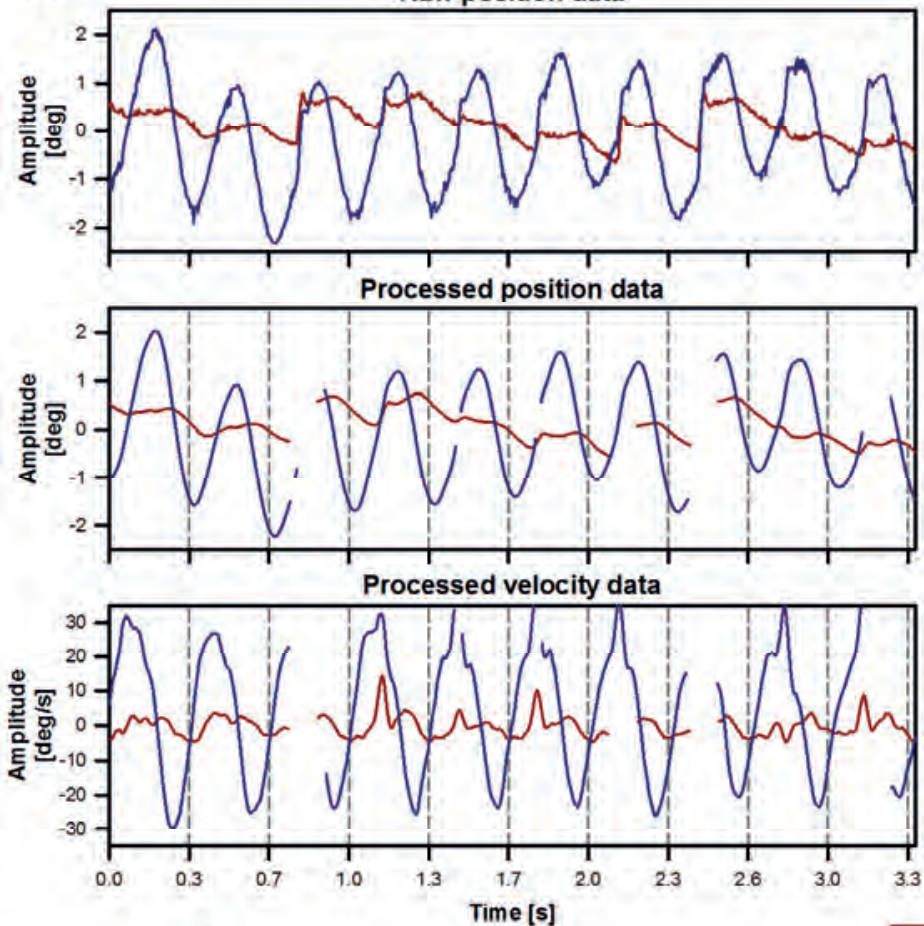

(B)
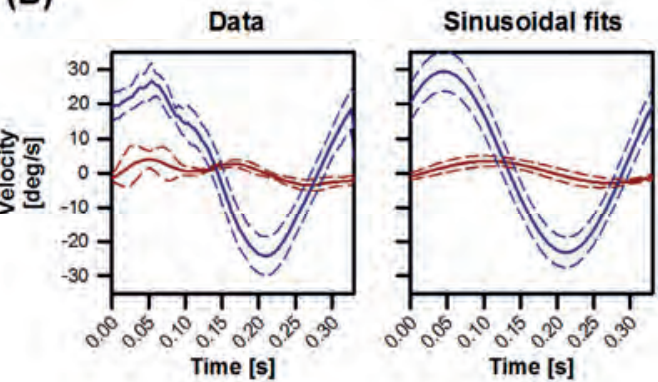

Figure 4.2 Illustration of eye movement data processing. The panels present eye movement data tracings for patient $\mathrm{S}$, gathered during the amplitude modulation experiments (frequency $3 \mathrm{~Hz}$, modulation strength corresponding to $75 \%$ of the patient's dynamic range). A Three steps are illustrated: raw eye position (e.g., before any processing was performed), processed eye position data (e.g., eye position data after low-pass filtering and after blinks and quick eye movements $>1,000 \%$ had been removed), and processed eye velocity data (e.g., obtained from the derivative of the processed eye position data). B Average cycle data \pm standard deviation (left) and their corresponding sinusoidal fits (right). 

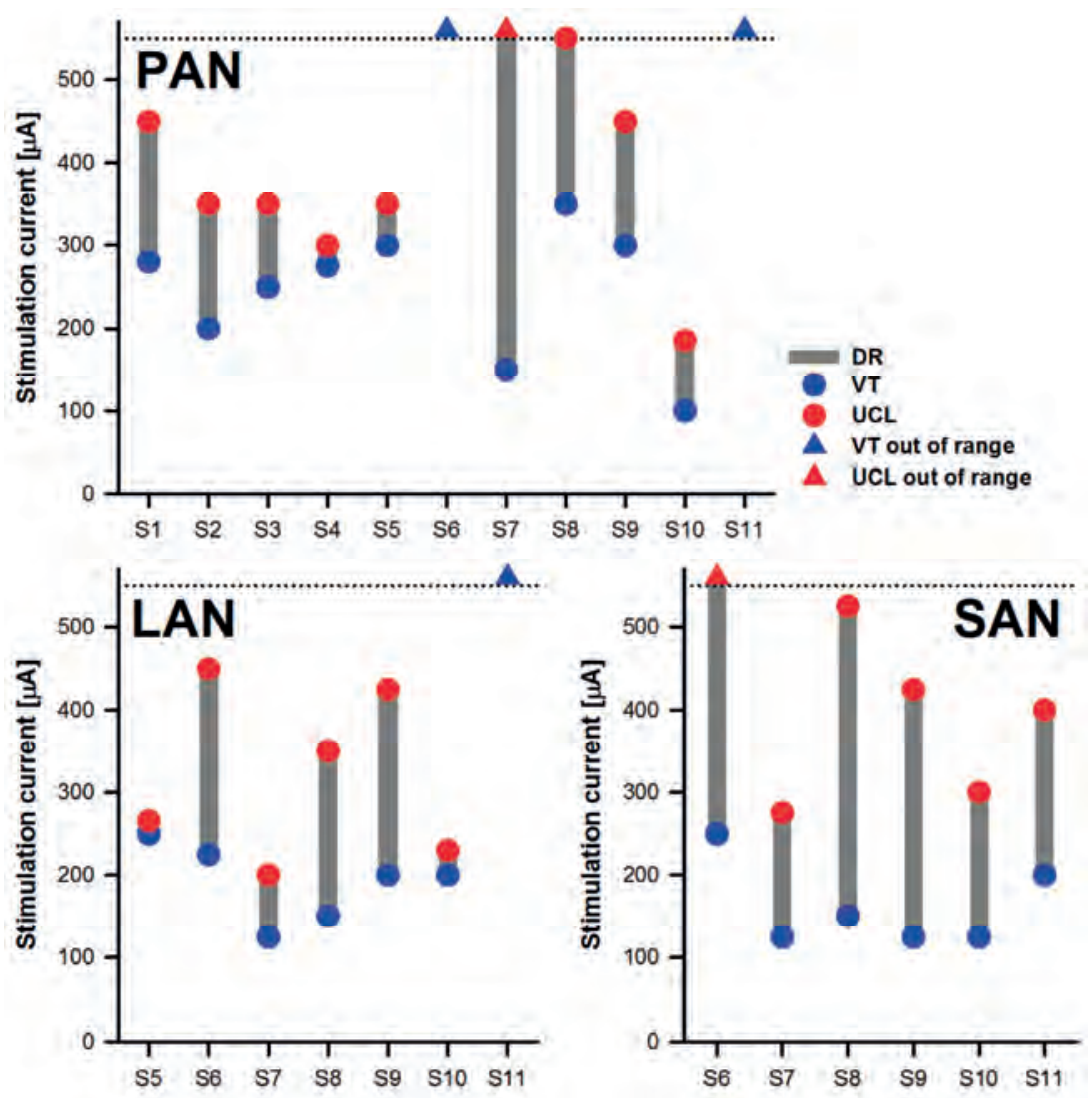

Figure 4.3 Vestibular thresholds (VT -blue circles), upper comfortable level (UCL - red circles) and corresponding dynamic range (DR - gray columns) are shown. Twenty-one out of the 24 available electrodes were responsive, and a dynamic range could be established. No response was obtained with 3 electrodes (blue triangles), even at the highest current tested $(550 \mu \mathrm{A}$, dotted lines in each panel). In 2 electrodes, no UCL could be determined, even at $550 \mu \mathrm{A}$ (red triangles). Note that in the case of the LAN electrode of subject S5, both the VT and the UCL were at the same current level $(250 \mu \mathrm{A})$ so the blue and red circles had to be slightly offset for visibility.

\section{Electrically elicited eye movements}

The main characteristics of the electrically elicited eye movements (total peak velocity and axis) are presented in Figure 4.4. The largest eye movements per electrode were observed in patient S7 for PAN $\left(26^{\circ} / \mathrm{s} ; 24.4-26.5^{\circ} / \mathrm{s}\right)$ and LAN $\left(32.7^{\circ} / \mathrm{s} ; 25.2-39.4^{\circ} / \mathrm{s}\right)$ and in patient S6 for SAN $\left(21.3^{\circ} / \mathrm{s} ; 18.5-23.4^{\circ} / \mathrm{s}\right)$. Consistent with the very variable dynamic ranges measured, the range of eye velocities was also very variable. Mean 
peak velocities per electrode across patients were $8.7 \pm 7.6^{\circ} / \mathrm{s}$ for PAN $(n=11)$, $13 \pm 12.5^{\circ} / \mathrm{s}$ for LAN $(n=6)$, and $11.9 \pm 6.6^{\circ} / \mathrm{s}$ for SAN $(n=5)$.

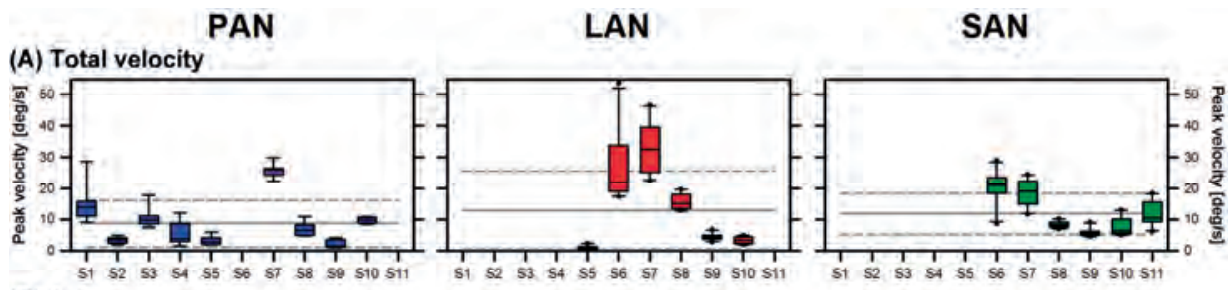

(B) Axis

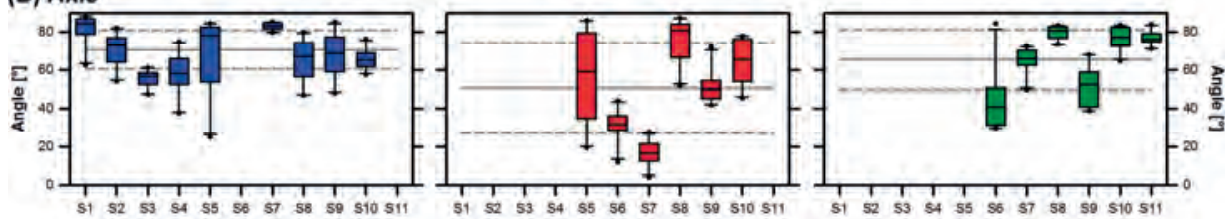

Figure 4.4 Main characteristics of electrically evoked eye movements. Each individual panel shows individual data for a given electrode (PAN: first column, blue box plots; LAN: middle column, red box plots; SAN: right col-umn, green box plots). Box plots indicate median values, 25th and 75th percentile values (colored box), 10th and 90th percentile values (error bars), and 5th and 95th percentile values (black circles). Mean values across patients, per electrode, are presented as gray solid lines ( \pm standard deviation, gray dashed lines). A Total peak velocity. B Axis (angle with respect to the horizontal plane) of the elicited eye movements, calculated over 10 consecutive cycles.

As expected from previous experiments, ${ }^{39,42}$ stimulation via 15 out of the 16 PAN and SAN electrodes resulted in eye movements with a predominantly vertical component. The mean angle for PAN stimulation was $70.6 \pm 10^{\circ}$ and of $65.4 \pm 15.8^{\circ}$ for SAN stimulation. Note, however, that for 1 SAN electrode, the angle was predominantly horizontal $\left(\mathrm{S} 6: 40.8^{\circ} ; 31.1-50.5^{\circ}\right)$. In contrast, stimulation of LAN electrodes resulted in a larger misalignment from the expected angle. The mean angle for LAN stimulation was $50.6 \pm 23.3^{\circ}$; only 2 out of the 6 LAN electrodes elicited eye movements predominantly in the horizontal plane (S6: $\left.31.7^{\circ}, 28.5-36^{\circ} ; \mathrm{S} 7: 16.5^{\circ}, 12.4-21.5^{\circ}\right)$.

The asymmetry index of the responses is presented in Figure 4.5. The most symmetrical responses were observed with PAN stimulation $(0.04 \pm 0.07)$. SAN stimulation $(0.08 \pm 0.15)$ and LAN stimulation $(0.14 \pm 0.11)$ showed a slightly higher asymmetry index. The less symmetrical responses per electrode were observed in patient S3 for PAN stimulation $(0.15,0.01-0.19)$, in patient S5 for LAN stimulation $(0.34,0.22-0.55)$, and in patient S10 for SAN stimulation $(0.20,-0.08$ to 0.24$)$. 


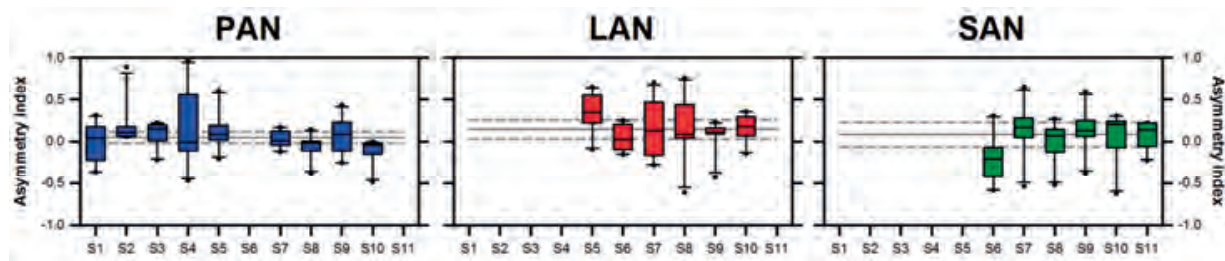

Figure 4.5 Asymmetry of the eye movements elicited via electrical stimulation of the PAN (left panel, blue box plots), the LAN (middle panel, red box plots), and the SAN (right panel, green box plots). Box plots indicate median values, 25th and 75th percentile values (colored box), 10th and 90th percentile values (error bars), and 5th and 95th percentile values (black circles). Mean values across patients, per electrode, are presented as gray solid lines ( \pm standard deviation, gray dashed lines). This index was calculated using best fits to excitatory/inhibitory stimulus half cycles (see Materials and Methods).

\section{Evoked percepts}

The first-reported perceptions for each electrode are summarized in Table 4.2. During PAN stimulation, sound was most frequently the first reported perception (6 out of 9). For LAN and SAN stimulation, diverse perceptions were reported, such as rotatory sensation, sound, tickling or pressure. The intensity of the perception reported for each electrode was also very variable. In general, the intensity of the reported percepts increased as the stimulation current increased. However, it was rarely consistent with evoked ocular responses.

Table 4.2 First-reported sensation upon stimulation of each of the vestibular electrodes.

\begin{tabular}{llll}
\hline & PAN & SAN & LAN \\
\hline S1 & sound, vertigo & n.a. & n.a. \\
S2 & sound & n.a. & n.a. \\
S3 & sound & n.a. & n.a. \\
S4 & sound & n.a. & n.a. \\
S5 & sound & n.a. & needle in the ear \\
S6 & none & rotatory sensation & rotatory sensation \\
S7 & eyes moving & eyes moving, 'tickling' sensation & ear 'tickling' \\
S8 & 'tickling' sensation & 'tickling' sensation & 'tickling' sensation \\
S9 & vibration, sound & vibration, sound & 'current-flow' sensation \\
S10 & rotatory sensation, & sound & Sound \\
S11 & none & pressure & Pressure \\
\hline
\end{tabular}

n.a. $=$ Not applicable. 


\section{Discussion}

The results presented here demonstrate that the concept of a unilateral vestibular implant is feasible in human patients. Motion information provided by the vestibular system is artificially mimicked by delivering a constant 'baseline' stimulation, which can be up- or downmodulated to evoke vestibulo-ocular responses. This allowed eliciting controlled eye movements in the 11 patients suffering from a BVL who received a vestibular implant. A particularly promising outcome of this study is that eye movements could be successfully evoked in a heterogeneous group of patients regarding the etiology of the deficit or the duration of the disease. This is particularly relevant since a significant concern was that vestibular dendrites could degenerate with time, precluding electrical stimulation of the vestibular nerve after long periods of sensory deprivation.

\section{Efficacy of electrical stimulation}

The efficacy of stimulation was very different across patients and across electrodes. Eye movements evoked by electrical stimulation of an ampullary nerve were expected to have an axis orthogonal to the plane of the corresponding semicircular canal. This was optimally achieved for PAN, LAN and SAN stimulation in 1 patient (S7). Moreover, in this case, mean peak eye velocities were $26,32.7$, and $19.1^{\circ}$ s for PAN, LAN, and SAN stimulation, respectively. This is a very promising finding, since in this case, evoked eye movements were within the range of compensatory eye movements previously reported during important dynamic daily activities, such as walking or running $\left(20-30 \%\right.$ s). ${ }^{51,54}$ Group results, however, showed some misalignment and lower mean peak eye velocities. Animal research reports suggest that adaptive processes could help improve the overall characteristics of the artificial eye movements in the long term. ${ }^{26,30,33}$ Furthermore, particular stimulation strategies (e.g.) incorporation of precompensatory 3D coordinate transformations, ${ }^{29}$ comodulation of the amplitude and pulse rate of the stimulation ${ }^{32}$ have also been suggested as possible alternatives to improve the characteristics of the electrically evoked vestibulo-ocular response.

It was expected that only very small eye movements could be elicited via electrodes with a narrow dynamic range (e.g., LAN responses for S10). However, surprisingly, eye movement responses were minimal in some cases with a relatively large dynamic range (e.g., LAN stimulation for S9). Despite this, patients still reported strong sensations related to vestibular stimulation (e.g., being pulled to the side or rotatory sensations). Such a dissociation between eye movement amplitude and sensation has also been reported by the team of the University of Washington. ${ }^{55}$ This suggests that electrical stimulation might be activating vestibular structures other than those 
involved in the generation of the vestibulo-ocular reflex. This finding deserves further investigation.

Three out of the 24 implanted electrodes were unresponsive. Some hypotheses can be put forward. In subject S6, the PAN electrode was unresponsive. A CT scan revealed an intraotic fracture line crossing the posterior ampulla and fibrosis filling the canal was found during surgery. Traumatic section or severe posttraumatic degeneration of the dendrites as well as fibrosis of the ampulla might drastically reduce the excitability of the vestibular nerve. The other 2 unresponsive electrodes were those implanted in the PAN and LAN of patient S11. This patient was suffering from DFNA9, an autosomal dominant nonsyndromic congenital disease due to $\mathrm{COCH}$ gene mutations. ${ }^{56}$ This adult-onset disorder is characterized by a progressive bilateral loss of cochlear and vestibular function. Severe loss of cochleovestibular nerve dendrite is a characteristic histological feature of this disease. ${ }^{57}$ This might preclude the success of intralabyrinthine electrical stimulation, due to the distance between the ampulla and the Scarpa ganglion. This hypothesis is reinforced by the fact that 7 out of the 9 vestibular electrodes of BVL patients diagnosed with DFNA9 (S9-S11) showed the smallest responses.

Finally, another factor that can significantly influence the effectiveness of electrical stimulation is optimal positioning of stimulating electrodes. Indeed, it has been observed that minimal position changes of the electrodes resulted in drastic changes of nystagmic responses. ${ }^{39}$ So far, peroperative stimulation under local and general anesthesia was performed to improve the electrode positioning. Peroperative vestibular electrically evoked action potentials could be an additional tool to improve the electrode positioning in the future. ${ }^{58}$

\section{Perception evoked via electrical stimulation}

Patients were actively requested to describe what they felt during the stimulation sessions. After unsuccessfully attempting to categorize the percepts described by the first implanted patients, raw description of any perception was documented. As can be seen in the results, described percepts were quite heterogeneous. This could be at least partially explained by the concomitant, spurious activation of nonvestibular neural structures due to current spread (i.e. the cochlear nerve, the branches of the glossopharyngeal nerve, and, to some extent, possibly also the vagal and the facial nerves).

\section{Extralabyrinthine versus intralabyrinthine electrode placement}

In 5 patients, electrodes $(n=6)$ were implanted close to vestibular nerve branches (extralabyrinthine approach), while in 6 patients, electrodes $(n=18)$ were implanted in the ampullae (intralabyrinthine approach). The first approach was initially chosen in 
the perspective of reducing the risk of inducing hearing loss (estimated around $4 \%$ as observed by Gacek and Gacek $^{59}$ in 252 neurectomies of the PAN for intractable benign paroxysmal positional vertigo). In contrast, the intralabyrinthine approach might allow better selectivity of the stimulation but the risk of inducing hearing loss in this case is still unclear. Results of animal studies are controversial: some demonstrate that intralabyrinthine electrode insertion with or without electrical stimulation impairs hearing in most of the cases, ${ }^{60-62}$ while others show that it is possible to preserve auditory and vestibular function. ${ }^{63}$ The results in human patients are not very encouraging in this respect. The group at the University of Washington has implanted 4 patients diagnosed with an intractable Menière's disease. They all had some preoperative residual vestibular and hearing function. An intralabyrinthine approach was used and postoperative hearing (reported only for 1 out of the 4 implanted patients) was almost totally lost. ${ }^{45,47}$ Nevertheless, there are reports showing that hearing is preserved after plugging of a dehiscent superior semicircular canal, plugging of the posterior superior semicircular canal for intractable benign paroxysmal positional vertigo, ${ }^{64,65}$ and plugging of the lateral superior semicircular canal in patients with severe Menière's disease. ${ }^{66}$ Since most patients suffering from a BVL have normal or near-normal hearing, it is crucial that the incidence of hearing loss upon vestibular implant surgery is thoroughly investigated and reported in the near future.

\section{Additional considerations}

In the natural vestibular system, motion is coded by modulation of the discharge rate of the spontaneous 'baseline' neural activity (i.e., number of spikes per second) of the vestibular nerve. For the lateral semicircular canal, the discharge rate increases with ampullopetal movements of the endolymph and decreases with movements of opposite direction. For example, for the horizontal semicircular canal, a head rotation in the direction of the canal (i.e., rightwards for the right ear) will result in an increase in the neuronal discharge rate. Conversely, a horizontal head rotation in a direction opposite to the canal (i.e., leftwards for the right ear) will result in a decrease in the discharge rate. The reverse is true for the vertical semicircular canals. ${ }^{67}$ In light of this physiological motion modulation scheme, discharge rate modulation has often been chosen as the stimulation method. ${ }^{29,68}$ However, in our experiments, eye movements were produced using amplitude modulation, not discharge rate modulation. This choice was motivated in a previous observation that in humans, discharge rate modulation resulted in smaller eye movements than amplitude modulation. ${ }^{44}$ Furthermore, our prototype vestibular implants are modified cochlear implants, which are designed to use amplitude modulation.

Consistent with previous data, ${ }^{26,31,46}$ some asymmetry was observed in the responses obtained upon stimulation with the majority of the electrodes. It is still unclear 
whether lack of symmetry will turn out to be a clinically relevant issue that will fundamentally limit the patient's benefit with the system. Furthermore, the vestibular system itself might be able to adapt to the electrical stimulus and improve the characteristics of the response with time. ${ }^{26,30,33}$ The use of different signal processing strategies (e.g., logarithmic vs. simple linear transfer functions) could also provide a potential solution.

\section{Conclusion}

These results confirm the feasibility of the concept of a vestibular implant for human use. We observed no medical complications related to the surgery or the device. Furthermore, the implant was successful at eliciting vestibulo-ocular responses even after long periods of implantation and in a very heterogeneous patient population. This, taken together with previous work, ${ }^{46}$ suggests that our objective of providing a first clinical tool to patients with a BVL might not be so far away.

\section{Acknowledgments}

This work was funded in part by the Seventh Framework Programme, Theme 3, Information and Communication Technologies, European Community CLONS (225929; CLOsed-loop Neural prostheses for vestibular disorderS), and by the INNOGAP proofof-principle fund (Unitec, University of Geneva). 


\section{References}

1. Guinand N, Boselie F, Guyot JP, Kingma H. Quality of life of patients with bilateral vestibulopathy. Ann Otol Rhinol Laryngol 2012;121:471-7.

2. Guinand N, Pijnenburg M, Janssen M, Kingma H. Visual acuity while walking and oscillopsia severity in healthy subjects and patients with unilateral and bilateral vestibular function loss. Arch Otolaryngol Head Neck Surg 2012;138:301-6.

3. Fuller PM, Jones TA, Jones SM, Fuller CA. Evidence for macular gravity receptor modulation of hypothalamic, limbic and autonomic nuclei. Neuroscience 2004;129:461-71.

4. Smith PF, Darlington CL. Personality changes in patients with vestibular dysfunction. Front Hum Neurosci 2013;7:678.

5. Smith PF, Zheng Y. From ear to uncertainty: vestibular contributions to cognitive function. Front Integr Neurosci 2013;7:84.

6. Zingler VC, Weintz E, Jahn K, Mike A, Huppert D, Rettinger N, Brandt T, Strupp M. Follow-up of vestibular function in bilateral vestibulopathy. J Neurol Neurosurg Psychiatry 2008;79:284-8.

7. Sun DQ, Ward BK, Semenov YR, Carey JP, Della Santina CC. Bilateral vestibular deficiency: quality of life and economic implications. JAMA Otolaryngol Head Neck Surg 2014;140:527-34.

8. Merfeld DM, Lewis RF. Replacing semicircular canal function with a vestibular implant. Curr Opin Otolaryngol Head Neck Surg 2012;20:386-92.

9. Gong W, Merfeld DM. Prototype neural semicircular canal prosthesis using patterned electrical stimulation. Ann Biomed Eng 2000;28:572-81.

10. Gong W, Merfeld DM. System design and performance of a unilateral horizontal semicircular canal prosthesis. IEEE Trans Biomed Eng 2002;49:175-81.

11. Della Santina CC, Migliaccio AA, Patel AH. A multichannel semicircular canal neural prosthesis using electrical stimulation to restore 3-d vestibular sensation. IEEE Trans Biomed Eng 2007;54:1016-30.

12. Valentin NS, Hageman KN, Dai C, Della Santina CC, Fridman GY. Development of a multichannel vestibular prosthesis prototype by modification of a commercially available cochlear implant. IEEE Trans Neural Syst Rehabil Eng 2013;21:830-9.

13. Rubinstein JT, Bierer S, Kaneko C, Ling L, Nie K, Oxford T, Newlands S, Santos F, Risi F, Abbas PJ, Phillips JO. Implantation of the semicircular canals with preservation of hearing and rotational sensitivity: a vestibular neurostimulator suitable for clinical research. Otol Neurotol 2012;33:789-96.

14. Jaeger A, Garnham C, Hessler R, Zimmerling M, Della Santina CC, Fridman G. Implantable vestibular prosthesis system with power saving mode including soft start and soft power-down. US2013184788A1; WO2013109678-A1; US8751012-B2; AU2013209844-A1.

15. Jaeger A, Garnham C, Hessler R, Zimmerling M, Della Santina CC, Fridman G. Vestibular implant system with low battery alert. WO2012177589-A1; US2013096654-A1; AU2012273147-A1; CA2838269-A1; CN103561812-A; EP2720750-A1.

16. Garnham C, Zimmerling M, Jaeger A. Vestibular implant system with internal and external motion sensors. WO2012012634-A1; US2012022616-A1; AU2011281029-A1; EP2595699-A1; AU2011281029B2; CN103153393-A; US2014005750-A1; AU2013270472-A1.

17. Merfeld DM, Rauch SD, Wall C, Weinberg M. Balance prosthesis. US Patent No US6546291 B2.

18. Pelizzone M, Perez Fornos A, Ranieri M, Cavuscens S. Device for electrical stimulation of neural and/or muscular tissue, has signal processing unit to transform input signal received from signal sensors into modu- lated electrical output signal to be treated by speech processor. WO2014118094A1; EP2762196-A1.

19. Cohen B, Suzuki Jl. Eye movements induced by ampullary nerve stimulation. Am J Physiol 1963;204:347-51.

20. Suzuki JI, Cohen B, Bender MB. Compensatory eye movements induced by vertical semicircular canal stimu- lation. Exp Neurol 1964;9:137-60.

21. Davidovics NS, Fridman GY, Chiang B, Della Santina CC. Effects of biphasic current pulse frequency, amplitude, duration, and interphase gap on eye movement responses to prosthetic electrical stimulation of the vestibular nerve. IEEE Trans Neural Syst Rehabil Eng 2011;19:84-94. 
22. Lewis RF, Gong W, Ramsey M, Minor L, Boyle R, Merfeld DM. Vestibular adaptation studied with a prosthetic semicircular canal. J Vestib Res 2002;12:87-94.

23. Merfeld DM, Gong W, Morrissey J, Saginaw M, Haburcakova C, Lewis RF. Acclimation to chronic constant-rate peripheral stimulation provided by a vestibular prosthesis. IEEE Trans Biomed Eng 2006;53:2362-72.

24. Merfeld DM, Haburcakova C, Gong W, Lewis RF. Chronic vestibulo-ocular reflexes evoked by a vestibular pros- thesis. IEEE Trans Biomed Eng 2007;54:1005-15.

25. Gong W, Haburcakova C, Merfeld DM. Vestibulo-ocular responses evoked via bilateral electrical stimulation of the lateral semicircular canals. IEEE Trans Biomed Eng 2008;55:2608-19.

26. Lewis RF, Haburcakova C, Gong W, Makary C, Merfeld DM. Vestibuloocular reflex adaptation investigated with chronic motion-modulated electrical stimulation of semicircular canal afferents. J Neurophysiol 2010;103:1066-79.

27. Saginaw MA, Gong W, Haburcakova C, Merfeld DM. Attenuation of eye movements evoked by a vestibular implant at the frequency of the baseline pulse rate. IEEE Trans Biomed Eng 2011;58: 2732-9.

28. Lewis RF, Haburcakova C, Gong W, Karmali F, Merfeld DM: Spatial and temporal properties of eye movements produced by electrical stimulation of semicircular canal afferents. J Neurophysiol 2012;108:1511-20.

29. Fridman GY, Davidovics NS, Dai C, Migliaccio AA, Della Santina CC. Vestibulo-ocular reflex responses to a multi- channel vestibular prosthesis incorporating a $3 \mathrm{D}$ coordinate transformation for correction of misalignment. J Assoc Res Otolaryngol 2010;11:367-81.

30. Dai C, Fridman GY, Chiang B, Davidovics NS, Melvin TA, Cullen KE, Della Santina CC. Cross-axis adaptation improves 3D vestibulo-ocular reflex alignment during chronic stimulation via a headmounted multichannel vestibular prosthesis. Exp Brain Res 2011;210:595-606.

31. Dai C, Fridman GY, Davidovics NS, Chiang B, Ahn JH, Della Santina CC. Restoration of 3D vestibular sensation in rhesus monkeys using a multichannel vestibular prosthesis. Hear Res 2011;281:74-83.

32. Davidovics NS, Fridman GY, Della Santina CC. Co-modulation of stimulus rate and current from elevated base- lines expands head motion encoding range of the vestibular prosthesis. Exp Brain Res 2012;218:389-400.

33. Dai C, Fridman GY, Chiang B, Rahman MA, Ahn JH, Davidovics NS, Della Santina CC. Directional plasticity rapidly improves 3D vestibulo-ocular reflex alignment in monkeys using a multichannel vestibular prosthesis. J Assoc Res Otolaryngol 2013;14:863-77.

34. Phillips C, Ling L, Oxford T, Nowack A, Nie K, Rubinstein JT, Phillips JO. Longitudinal performance of an implantable vestibular prosthesis. Hear Res 2015;322:200-11.

35. Thompson LA, Haburcakova C, Gong W, Lee DJ, Wall C 3rd, Merfeld DM, Lewis RF. Responses evoked by a vestibular implant providing chronic stimulation. J Vestib Res 2012;22:11-5.

36. Lewis RF, Haburcakova C, Gong W, Lee D, Merfeld D. Electrical stimulation of semicircular canal afferents affects the perception of head orientation. J Neurosci 2013;33:9530-5.

37. Mitchell DE, Dai C, Rahman MA, Ahn JH, Della Santina CC, Cullen KE. Head movements evoked in alert rhesus monkey by vestibular prosthesis stimulation: implications for postural and gaze stabilization. PLoS One 2013;8:e78767.

38. Kos MI, Feigl G, Anderhuber F, Wall C, Fasel JH, Guyot JP. Transcanal approach to the singular nerve. Otol Neurotol 2006;27:542-6.

39. Wall C 3rd, Kos MI, Guyot JP. Eye movements in response to electric stimulation of the human posterior ampullary nerve. Ann Otol Rhinol Laryngol 2007;116:369-74.

40. Feigl G, Kos I, Anderhuber F, Guyot JP, Fasel J. Development of surgical skill with singular neurectomy using human cadaveric temporal bones. Ann Anat 2008;190:316-23.

41. Feigl GC, Fasel JH, Anderhuber F, Ulz H, Rienmuller R, Guyot JP, Kos IM. Superior vestibular neurectomy: a novel transmeatal approach for a denervation of the superior and lateral semicircular canals. Otol Neurotol 2009;30:586-91.

42. Guyot JP, Sigrist A, Pelizzone M, Feigl GC, Kos MI. Eye movements in response to electrical stimulation of the lateral and superior ampullary nerves. Ann Otol Rhinol Laryngol 2011;120:81-7. 
43. van de Berg R, Guinand N, Guyot JP, Kingma H, Stokroos RJ. The modified ampullar approach for vestibular implant surgery: feasibility and its first application in a human with a long-term vestibular loss. Front Neurol 2012;3:18.

44. Guyot JP, Sigrist A, Pelizzone M, Kos MI. Adaptation to steady-state electrical stimulation of the vestibular system in humans. Ann Otol Rhinol Laryngol 2011;120:143-9.

45. Golub JS, Ling L, Nie K, Nowack A, Shepherd SJ, Bierer SM, Jameyson E, Kaneko CR, Phillips JO, Rubinstein JT. Prosthetic implantation of the human vestibular system. Otol Neurotol 2014;35: 136-47.

46. Perez Fornos A, Guinand N, van de Berg R, Stokroos R, Micera S, Kingma H, Pelizzone M, Guyot JP. Artificial balance: restoration of the vestibulo-ocular reflex in humans with a prototype vestibular neuroprosthesis. Front Neurol 2014;5:66.

47. Phillips C, Defrancisci C, Ling L, Nie K, Nowack A, Phillips JO, Rubinstein JT. Postural responses to electrical stimulation of the vestibular end organs in human subjects. Exp Brain Res 2013;229:181-95.

48. Shannon RV, Zeng FG, Kamath V, Wygonski J, Ekelid M. Speech recognition with primarily temporal cues. Science 1995;270:303-4.

49. Haslwanter T. Mathematics of three-dimensional eye rotations. Vision Res 1995;35:1727-39.

50. Robinson DA. A method of measuring eye movement using a scleral search coil in a magnetic field. IEEE Trans Biomed Eng 1963;10:137-45.

51. Crane BT, Demer JL. Human gaze stabilization during natural activities: translation, rotation, magnification, and target distance effects. J Neurophysiol 1997;78:2129-44.

52. Weber KP, MacDougall HG, Halmagyi GM, Curthoys IS. Impulsive testing of semicircular-canal function using video-oculography. Ann NY Acad Sci 2009;1164:486-91.

53. Bartl K, Lehnen N, Kohlbecher S, Schneider E. Head impulse testing using video-oculography. Ann NY Acad Sci 2009;1164:331-3.

54. Grossman GE, Leigh RJ, Bruce EN, Huebner WP, Lanska DJ. Performance of the human vestibuloocular reflex during locomotion. J Neurophysiol 1989;62:264-72.

55. Rubinstein JT. Human longitudinal studies of electrical stimulation of the vestibular periphery. 13th Int Conf Cochlear Implants Other Implantable Auditory Technol, Munich, 2014, pp KN4-4.

56. Manolis EN, Yandavi N, Nadol JB Jr, Eavey RD, McKenna M, Rosenbaum S, Khetarpal U, Halpin C, Merchant SN, Duyk GM, et al. A gene for non-syndromic autosomal dominant progressive postlingual sensorineural hearing loss maps to chromosome 14q12-13. Hum Mol Genet 1996;5:1047-50.

57. Merchant SN, Linthicum FH, Nadol JB Jr. Histopathology of the inner ear in DFNA9. Adv Otorhinolaryngol 2000;56:212-7.

58. Nie K, Bierer SM, Ling L, Oxford T, Rubinstein JT, Phillips JO. Characterization of the electrically evoked compound action potential of the vestibular nerve. Otol Neurotol 2011;32:88-97.

59. Gacek RR, Gacek MR. Results of singular neurectomy in the posterior ampullary recess. ORL J Otorhinolar- yngol Relat Spec 2002;64:397-402.

60. Tang S, Melvin TA, Della Santina CC. Effects of semicircular canal electrode implantation on hearing in chin- chillas. Acta Otolaryngol 2009;129:481-6.

61. Dai C, Fridman GY, Della Santina CC. Effects of vestibular prosthesis electrode implantation and stimulation on hearing in rhesus monkeys. Hear Res 2011;277:204-10.

62. Tran H, de Waele C, Beraneck M, Vassias I, Gioanni H, Huy PT, Herman P, Vidal PP, Kania RE. Auditory outcomes after implantation and electrical stimulation of the lateral ampullar nerve in guinea pig. Ear Hear 2012;33:118-23.

63. Bierer SM, Ling L, Nie K, Fuchs AF, Kaneko CR, Oxford T, Nowack AL, Shepherd SJ, Rubinstein JT, Phillips JO. Auditory outcomes following implantation and electrical stimulation of the semicircular canals. Hear Res 2012;287:51-6.

64. Limb CJ, Carey JP, Srireddy S, Minor LB. Auditory function in patients with surgically treated superior semi- circular canal dehiscence. Otol Neurotol :2006;27:969-80.

65. Agrawal SK, Parnes LS. Human experience with canal plugging. Ann NY Acad Sci 2001;942:300-5.

66. Charpiot $A$, Rohmer $D$, Gentine $A$. Lateral semicircular canal plugging in severe Ménière's disease: a clinical prospective study about 28 patients. Otol Neurotol 2010;31:237-40. 
67. Goldberg JM, Fernandez C. Physiology of peripheral neurons innervating semicircular canals of the squirrel monkey. I. Resting discharge and response to constant angular accelerations. J Neurophysiol 1971;34:635-60.

68. Lewis RF, Haburcakova C, Gong W, Makary C, Merfeld DM. Vestibulo-ocular reflex adaptation investigated with chronic motion-modulated electrical stimulation of semicircular canal afferents. J Neurophysiol 2010;103:1066-79. 



\section{Chapter 5 \\ 5}

\section{Restoring visual acuity in dynamic conditions with a vestibular implant}

Nils Guinand, Raymond Van de Berg, Samuel Cavuscens, Robert Stokroos, Maurizio Ranieri, Marco Pelizzone, Herman Kingma, Jean-Philippe Guyot, Angélica Pérez Fornos 


\section{Abstract}

Vestibular implants are devices designed to rehabilitate patients with a bilateral vestibular loss (BVL). These patients lack a properly functioning vestibulo-ocularreflex (VOR), which impairs gaze stabilization abilities and results in an abnormal loss of visual acuity (VA) in dynamic situations (i.e., severely limiting the patient's ability to read signs or recognize faces while walking). We previously demonstrated that the VOR can be artificially restored in a group of BVL patient sfitted with a prototype vestibular implant. This study was designed to investigate whether these promising results could be translated to a close-to-reality task, significantly improving VA abilities while walking. Six BVL patients previously implanted with a vestibular implant prototype participated in the experiments. VA was determined using Sloan letters displayed on a computer screen, in four conditions: (1) with the patient standing still without moving (static), (2) while the patient was walking on a treadmill at constant speed with the vestibular implant prototype turned off (systemOFF), (3) while the patient was walking on a treadmill at constant speed with the vestibular implant prototype turned on providing coherent motion information (systemONmotion), and (4) a "placebo" condition where the patient was walking on a treadmill at constant speed with the vestibular implant prototype turned on providing reversed motion information (systemONsham). The analysis (one-way repeated measures analysis of variance) revealed a statistically significant effect of the test condition $[F=(3,12) 30.5, p<0.001]$. Significant decreases in VA were observed with the system OFF condition when compared to the static condition (Tukey post-hocp<0.001). When the vestibular implant was turned on, delivering pertinent motion information (systemONmotion) the VA improved to close to normal values. The improvement disappeared in the placebo condition (systemONsham) and VA-values also dropped significantly in this condition (Tukeyposthocp $<0.001$ ). These results are a significant step forward in the field, demonstrating for the first time in humans that gaze stabilization abilities can be restored with a vestibular implant prototype. The vestibular implant shows considerable promise of being the first-ever effective therapeutic alternative for patients with a BVL in the near future. 


\section{Introduction}

The vestibular implant is a device designed to artificially restore the vestibular function using motion modulated electrical stimulation of the peripheral vestibular system. In the past two decades, several groups have demonstrated that three key aspects of vestibular function: the vestibulo-ocular, ${ }^{1-3}$ specific postural responses, ${ }^{4}$ as well as vestibular percepts ${ }^{5}$ can be artificially elicited and restored using a vestibular implant. This confirms that it is possible to effectively transmit motion information to the central nervous with such a device. The vestibular implant concept could be applicable in cases of severe bilateral loss of the vestibular function (BVL), a very disabling and poorly recognized condition for which no effective treatment exists. ${ }^{6,7}$ Our group has recently demonstrated partial restoration of the VOR in a group of BVL patients fitted with a prototype vestibular implant, using motion modulated electrical stimulation of the vestibular nerve. ${ }^{3}$ In certain cases the velocity of the elicited eye movements was within the range of compensatory eye movements observed in healthy subjects during walking or running ${ }^{8,5}$ and the electrically evoked VOR displayed a similar frequency response as the physiological VOR. ${ }^{9}$ These results can be considered as a milestone in the development of a vestibular implant, confirming in humans pioneering findings obtained in animal research. ${ }^{2,10}$ The next fundamental question was whether this artificial vestibular reflex could be useful to improve performance on a clinically significant, more complex task. The majority of patients diagnosed with a BVL describe a clinical manifestation consisting of blurred vision or oscillopsia. For example, they experience difficulties recognizing faces or reading signs while walking. This is mainly attributed to the loss of the vestibular reflexes, in particular of the VOR that holds a crucial role in the mechanism of gaze stabilization. In the clinic, this can be quantified as a pathological drop of visual acuity (VA) in dynamic conditions compared to a static condition. ${ }^{11}$ Some testing protocols use passive, unpredictable high velocity yaw or pitch head movements as stimuli for the dynamic condition. They have demonstrated high sensitivity in revealing a BVL. ${ }^{12,13}$ The vestibular implant is a device designed to artificially restore the vestibular function using motion modulated electrical stimulation of the peripheral vestibular system. In the past two decades, several groups have demonstrated that three key aspects of vestibular function: the vestibulo-ocular reflex, ${ }^{1-3,14}$ specific postural responses, ${ }^{4}$ as well as vestibular percepts ${ }^{5}$ can be artificially elicited and restored using a vestibular implant. This confirms that it is possible to effectively transmit motion information to the central nervous with such a device. The vestibular implant concept could be applicable in cases of severe bilateral loss of the vestibular function $(B V L)$, a very disabling and poorly recognized condition for which no effective treatment exists. ${ }^{6,7}$ Our group has recently demonstrated partial restoration of the VOR in a group of BVL patients fitted with a prototype vestibular implant, using motion modulated electrical stimulation of the vestibular nerve. ${ }^{3}$ In certain cases the 
velocity of the elicited eye movements was within the range of compensatory eye movements observed in healthy subjects during walking or running ${ }^{5,8}$ and the electrically evoked VOR displayed a similar frequency response as the physiological VOR. ${ }^{9}$ These results can be considered as a milestone in the development of a vestibular implant, confirming in humans pioneering findings obtained in animal research. ${ }^{2,10}$ The next fundamental question was whether this artificial vestibular reflex could be useful to improve performance on a clinically significant, more complex task. The majority of patients diagnosed with a BVL describe a clinical manifestation consisting of blurred vision or oscillopsia. For example, they experience difficulties recognizing faces or reading signs while walking. This is mainly attributed to the loss of the vestibular reflexes, in particular of the VOR that holds a crucial role in the mechanism of gaze stabilization. In the clinic, this can be quantified as a pathological drop of visual acuity (VA) in dynamic conditions compared to a static condition. ${ }^{11}$ Some testing protocols use passive, unpredictable high velocity yaw or pitch head movements as stimuli for the dynamic condition. They have demonstrated high sensitivity in revealing a BVL. ${ }^{12,13}$

However, a drawback of these methods is that the stimuli used are not physiological. Another more representative method of the everyday challenge faced by BVL patients is the evaluation of VA while walking on a treadmill at controlled velocities. ${ }^{15}$ This original method has been shown to be reliable and sensitive to detect BVL, ${ }^{16}$ even at low walking speeds of $2 \mathrm{~km} / \mathrm{h}^{11}$ Demonstrating the restoration of gaze stabilization abilities in BVL patients, particularly in a close-to-reality task would constitute a significant step forward in the rehabilitation of vestibular deficits. In the present study, we investigated whether motion-modulated electrical stimulation could be used to normalize VA abilities while walking in a group of BVL patients chronically implanted with a vestibular implant.

\section{Materials and methods}

\section{Patients and device}

Twelve BVL patients, unilaterally or bilaterally deaf, were recruited the Service of Otorhinolaryngology and Head and Neck Surgery at the Geneva University Hospitals and at the Division of Balance Disorders at the Maastricht University Medical Center. Strict inclusion criteria were implemented, and have been described in detail previously. ${ }^{5}$ Patients were fitted with prototype vestibular implants. These devices consisted of a modified cochlear implant (MED-EL, Innsbruck, Austria) that provided, in addition to the cochlear array, extra-cochlear electrodes for "vestibular" stimulation. Extra- or intra-labyrinthine implantation of the electrodes in the vicinity of the ampullary branches of the vestibular nerve, as previously described, ${ }^{17,18}$ was 
performed.The vestibular implant was activated no earlier than 3 weeks after surgery. Six out of the twelve implanted patients were available for dynamic VA experiments presented in this paper(Table 5.1). A regular cochlear implant processor (Tempo+MED-EL, Innsbruck, Austria) was used to control the electrical stimulation delivered by the selected electrode using a customized transformation unit connected to the auxiliary input of the processor. ${ }^{19}$ Angular head motion was captured with a three-axis gyroscope (device based on the sensor LYPR540AH; ST Micro-electronics; Geneva, Switzerland), fixed to the patient's head using a customized helmet.

Table 5.1 Demographics and implantation details of participating patients.

\begin{tabular}{|c|c|c|c|c|c|c|c|}
\hline Patient & Sex & Etiology & $\begin{array}{c}\text { Age (at } \\
\text { implantation) }\end{array}$ & $\begin{array}{c}\text { Implantation } \\
\text { year }\end{array}$ & $\begin{array}{c}\text { Active } \\
\text { electrode }\end{array}$ & $\begin{array}{l}\text { Surgical } \\
\text { approach }\end{array}$ & $\begin{array}{c}\text { Baseline stimulation } \\
\text { amplitude (Dynamic } \\
\text { range; } \mu \mathrm{A} \text { ) }\end{array}$ \\
\hline S1 & $M$ & Idiopathic & 68 & 2007 & PAN & EL & $360(170)$ \\
\hline S2 & M & $\begin{array}{l}\text { Congenital/ } \\
\text { idiopathic }\end{array}$ & 46 & 2008 & PAN & EL & $300(100)$ \\
\hline S3 & $\mathrm{F}$ & Traumatic & 67 & 2013 & SAN & IL & $410(300)$ \\
\hline S4 & $\mathrm{F}$ & Meningitis & 48 & 2012 & SAN & IL & $200(180)$ \\
\hline S5 & M & DFNA9 & 66 & 2013 & PAN & IL & $120(80)$ \\
\hline S6 & $\mathrm{M}$ & Traumatic & 53 & 2015 & SAN & IL & $350(450)$ \\
\hline
\end{tabular}

PAN, posterior ampullary nerve; EL, extra-labyrinthine; SAN, superior ampullary nerve; IL, intra-labyrinthine.

\section{Electrical stimulation}

As the predominant components of head movements during walking are pitch and vertical translation, ${ }^{8}$ electrodes in the vicinity of the posterior (PAN) or superior (SAN) ampullary nerves were selected to deliver motion information using electrical currents. Theoretically, stimulation of these vertical vestibular nerve branches should generate vertical compensatory eye movements. ${ }^{20}$ Only one vestibular electrode was active during the experiments and all cochlear electrodes were turned off. As already described in previous publications, to generate bidirectional eye movements (i.e., upwards and downwards when stimulating the vertical nerve branches) when using unilateral vestibular stimulation, it was necessary to first restore and maintain a baseline stimulation of the vestibular nerve., ${ }^{3,5,21}$ In this study, we chose a supra physiological baseline stimulation profile consisting of trains of biphasic, chargebalanced (200 $\mathrm{\mu s} /$ phase) pulses presented at a rate of 400 pulses per s. These stimulation parameters were selected because they have proved to be particularly effective for activating the vestibular system in our particular setting. The amplitude of the baseline stimulation was set in the middle of the dynamic range measured for each patient. ${ }^{5}$ Once in the adapted state, ${ }^{21}$ the motion signal captured by the head mounted gyroscope could be used to up- and down-modulate the amplitude of the train of pulses delivered via the SAN or PAN vestibular electrodes. 
We arbitrarily chose to implement a simple linear transfer function between measured pitch head velocity and electrical stimulation delivered via the SAN or PAN electrode. It was defined based on the previously measured dynamic range and eye movement response characterized for each subject. ${ }^{5}$ Amaximumof $85 \%$ of each patient's dynamic range was used to code for $30 \% / \mathrm{s}$, based on previous data on the main characteristics of the VOR during locomotion. ${ }^{8}$ For safety reasons, maximum stimulation delivered was hard coded to be limited to $90 \%$ of the patient's dynamic range, to avoid excessively high currents in case any abrupt, rapid head movement occurred.

\section{Visual acuity measurements}

During the experiments, patients had to read aloud sequences of Sloan optotypes (CDHKNORSVZ) of decreasing size displayed in a random order one at a time on a computer screen (15 inches). The screen was positioned at eyes' height, $2.8 \mathrm{~m}$ in front of the patient. The sequence started with a five letters presentation at $1 \log M A R$ (logarithm of the Minimum Angle of Resolution). If the letter recognition rate was above chance $(>10 \%)$, the letter size was decreased by a step of 0.1 logMAR and five new letters were presented one at a time. The same procedure was continued until the recognition rate for a given letter size dropped below chance $(\leq 10 \%)$. Two almost identical additional runs were repeated.

The experiments were carried out on a treadmill in four conditions: (1) with the patient standing still (static), (2) while the patient was walking at a constant speed with the vestibular implant turned off (systemOFF), (3) while the patient was walking at a constant speed with the vestibular implant turned on and delivering coherent motion information to the patient's vestibular nerve (i.e., amplitude of the baseline stimulation modulated using the signal coming from the pitch axis of the gyroscope; systemON $\mathrm{N}_{\text {motion }}$ ), and (4) while the patient was walking at a constant speed with the vestibular implant turned on and delivering incoherent motion information to the patient's vestibular nerve (i.e., amplitude of the baseline stimulation modulated using the reversed signal coming from the pitch axis of the gyroscope; system $\mathrm{ON}_{\text {sham }}$ ). Walking speed was set between 2 and $4 \mathrm{~km} / \mathrm{h}$, at the maximum where the patient felt safe and could walk without holding the handrails in the systemOFF condition. Once the maximum safe speed for the patient was selected, it was kept constant for all the dynamic conditions. The order in which each of the three dynamic conditions was conducted was determined using a Latin square design, randomized across patients. All experiments were written in MATLAB (R2010a; Mathworks, Natick MA, USA) using the Psychtoolbox. ${ }^{22-24}$

Raw data, expressed in logMAR, were converted to decimal VA-values and normalized to $V A$ obtained in static conditions. ${ }^{25} \mathrm{~A}$ one-way repeated measures analysis of variance (ANOVA) was conducted to compare VA across conditions. All statistical 
analyses were performed with the statistics package for SigmaPlot 13.0 (Systat Software, Inc., Chicago, IL, USA).

\section{Ethics statement}

All subjects gave written informed consent in accordance with the Declaration of Helsinki. Approval of the ethical committees of the Geneva University Hospitals (NAC 11-080) and of the Maastricht University Medical Center (NL36777.068.11/METC 112-031) was obtained.

\section{Results}

All patients were able to complete the procedure at their own maximum safe walking velocity $(2-4 \mathrm{~km} / \mathrm{h})$. Absolute VA-values obtained in each condition are presented in Table 5.2. Please note that lower logMAR-values indicate better scores.

Table 5.2 Absolute VA-values obtained in each condition per patient [logMAR].

\begin{tabular}{lccccc}
\hline Patient & Static VA & SystemOFF & SystemON $_{\text {motion }}$ & SystemON $_{\text {sham }}$ & MSWV \\
\hline S1 & 0.04 & 0.18 & 0.04 & 0.14 & 2 \\
S2 & -0.07 & 0.19 & 0.04 & 0.13 & 4 \\
S3 & -0.21 & 0.07 & -0.14 & -0.02 & 3 \\
S4 & -0.34 & -0.17 & -0.31 & -0.31 & 4 \\
S5 & -0.13 & 0.00 & -0.11 & -0.05 & 4 \\
S6 & 0.06 & 0.31 & 0.22 & 0.33 & 3 \\
\hline
\end{tabular}

Maximum safe walking velocities [MSWV $(\mathrm{km} / \mathrm{h})]$ in each case are also indicated.

Compared to the static condition, all six patients experienced a drop in VA while walking on the treadmill in the systemOFF condition, ranging from 0.13 to 0.28 logMAR in absolute value (a loss of 0.1 logMAR corresponds to a loss of one line on a standard letter chart used for the measurement of the VA). In the systemON $\mathrm{N}_{\text {motion }}$ condition, the VA improved in all patients compared to the systemOFF condition, and even equaled the value of the static condition in one patient (S1). The VA differences between the static and the systemON $\mathrm{N}_{\text {motion }}$ conditions ranged from 0 to $0.16 \log \mathrm{MAR}$. The VA differences between the static and the system $O \mathrm{~N}_{\text {motion }}$ conditions were smaller than the VA differences between the static and the system $\mathrm{N}_{\text {sham }}$ conditions in all six patients. The VA differences between the static and the system $\mathrm{ON}_{\text {sham }}$ conditions were smaller than the VA difference between the static and the systemOFF conditions, except for S6. The range of the VA differences between the static and the systemON $\mathrm{N}_{\text {sham }}$ condition was 0.03 to $0.28 \log \mathrm{MAR}$. 
Normalization of individual VA scores to values obtained in the static condition allows a better representation and facilitates comparison of the previously mentioned trends for each patient (Figure 5.1A). The ANOVA analysis showed a statistically significant difference between conditions $\left[F_{(3,12)}=30.49, P<0.001\right]$. Post-hoc tests (Tukey) revealed a significant $(P<0.001)$ increase of the VA loss in the system OFF and in the systemON $\mathrm{N}_{\text {sham }}$ conditions, compared to the static and the systemON $\mathrm{N}_{\text {motion }}$ conditions. No significant differences were found, either between the static and the system $\mathrm{N}_{\text {motion }}$ conditions, or between the system OFF and the system $\mathrm{ON}_{\text {sham }}$ conditions (see Figure 5.1B).
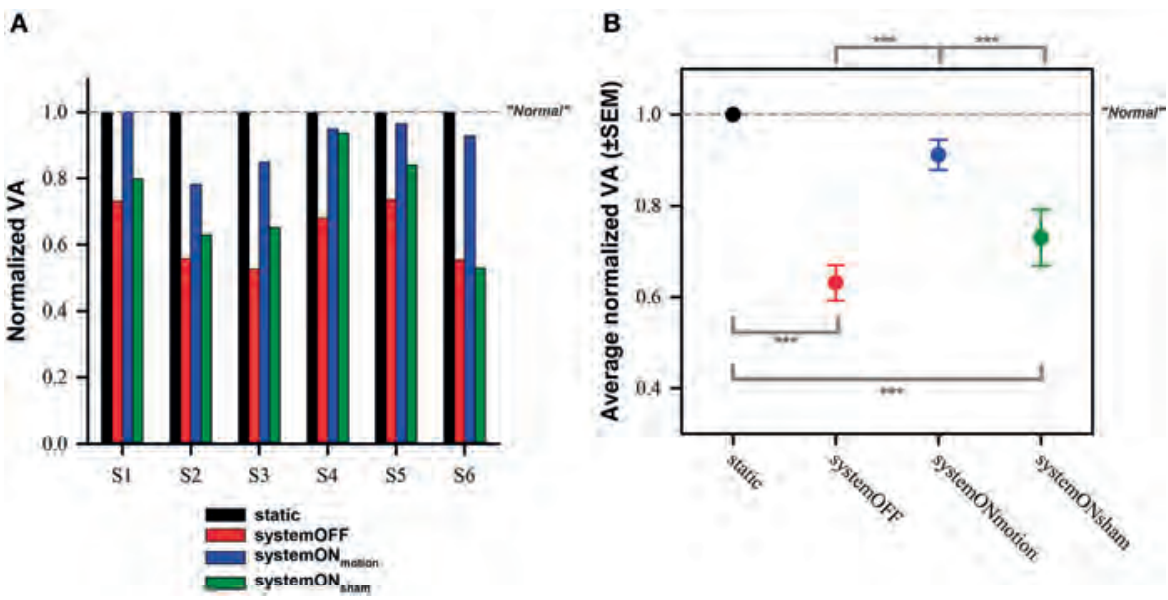

Figure 5.1 Normalized visual acuity results. (A) Individual results obtained in the dynamic conditions (colored bars; red-systemOFF, blue-system $O N_{\text {motion, }}$ green-systemON $\mathrm{N}_{\text {sham }}$ ) for the six participating subjects, normalized to results obtained in the static condition (black bar). (B) Average normalized results $\left( \pm\right.$ standard error of the mean, SEM). ${ }^{* * *}$ Indicates significant differences between conditions in the post-hoc tests (Tukey). Dotted gray lines indicate theoretical performance of "normal" subjects (i.e., no loss of visual acuity in dynamic situations).

\section{Discussion}

These results clearly indicate that the vestibular implant successfully transfers motion information to the brain, leading to restoration of VA abilities in a dynamic situation (walking in standardized conditions). This represents the first demonstration of functional rehabilitation using the concept of motion- modulated electrical stimulation of the vestibular nerve in humans, and therefore constitutes a fundamental milestone in the field.

To investigate whether it was possible to improve gaze stabilization abilities in BVL patients wearing a prototype vestibular implant, a protocol representative of one of 
the most common complaints was implemented. When walking, patients with a BVL present a significant loss of VA which is correlated with the presence of oscillopsia. ${ }^{11}$ Using motion modulated electrical stimulation, the VA measured in dynamic conditions (i.e., while walking) was significantly improved to a value close to that measured in static conditions in all six patients tested. The fact that the VA improvement decreased significantly in the system $\mathrm{ON}_{\text {sham }}$ condition further confirms that observed improvements were due to the properly functioning vestibular implant. Interestingly however, the VA loss observed in the systemON $\mathrm{N}_{\text {sham }}$ condition was slightly smaller than that observed in the systemOFF condition (non-significant), suggesting that some useful motion information could still have been extracted by the brain in the system $\mathrm{ON}_{\text {sham }}$ condition where the gain was reversed.

It is generally accepted that the drop of VA measured in dynamic conditions in patients suffering from a BVL is due to a poorly functioning (or absent) VOR, which is generally considered the main vestibular mechanism involved in gaze stabilization. Initially, we wanted to quantify the artificially generated VOR during the VA task in dynamic conditions in order to demonstrate that any measured improvements would be due to the restoration of this reflex with our vestibular implant prototype. We attempted recording eye movements while we measured the VA in dynamic conditions using a fast 2D video- oculography system (EyeSeeCam VOG; Munich, Germany), but were not successful in achieving precise recordings. In order to avoid artifacts due to goggle slippage, the goggles had to be very tightly adjusted. This was too painful to patients after just a few minutes, not giving enough time to complete the task. Furthermore, the tightly fixed glasses also disrupted the visual abilities of patients, especially at near-threshold values. As a consequence, we decided not to record eye movements during VA measurements. However, in an attempt to better understand how the magnitude of the electrically evoked VOR influenced the VA results we decided to compare the latter with previously presented results of the artificial VOR measured in static conditions. ${ }^{5}$ Surprisingly, we found no correlation between the magnitude of the evoked VOR and the observed improvements in the systemON $\mathrm{N}_{\text {motion }}$ condition. This could of course be due to the small sample size of the study. However, it could also suggest other vestibular mechanisms could also be substantially contributing to gaze stabilization. A first hypothesis is that, by electrically delivering motion information to the vestibular nerve, other vestibular reflexes are also activated. Indeed, although it was not systematically documented in this study, during the static artificial VOR measurements we observed that in some cases sinusoidal head movements were evoked in parallel to eye movements. Moreover, these head movements were phase locked with the sinusoidal electrical stimulus. This strongly suggests that the vestibulo-collic and the vestibulo-spinal pathways were also activated during our experiments. This hypothesis is supported by observations of other research teams. For example, postural responses have been reported upon electrical stimulation of the ampullas ${ }^{4}$ and even by using motion modulated 
stimulation delivered by intracochlear electrodes of a regular cochlear implant. ${ }^{26}$ More recently, direct activation of vestibular reflexes upon electrical stimulation delivered through the intracochlear array of the cochlear implant has also been demonstrated. ${ }^{27}$

Future research efforts will be devoted to a more comprehensive evaluation of vestibular function, well-beyond the VOR. For example, a matter of particular interest will be to better understand whether the activation of the vestibulo-collic pathway results from current spread to the otolithic organs, or whether the role of the semicircular canals in the control of posture has been underestimated. In addition, up to now all our experiments have been carried out while activating a single electrode at a time. Future experiments we will assess the simultaneous use of multiple vestibular electrodes (in contact with all three ampullary nerves) for integration of 3D angular motion information. This will imply the development of more complex stimulation parameters and strategies. Another important aspect of future developments will involve the refinement of the electrode design and of the surgical insertion techniques to optimize electrode positioning (i.e., selectivity of the stimulation), while preserving any pre-existing auditory and residual vestibular function. This is of crucial importance as the majority of patients with a BVL have normal or only mild hearing loss. In addition, to warrant successful translation of vestibular implants to the clinic, surgical procedures should be simplified and standardized as much as possible in order to become accessible to most of otologists. Finally, a unique aspect of the vestibular implant is that it is the first experimental setup that allows activating the vestibular system exclusively, without the unwanted contribution of other sensory modalities (e.g., vision, proprioception) that intervene in the complex activities mediated by balance. We expect thus that basic research studies with this device will open new doors increasing our fundamental knowledge on the physiology of the vestibular system and its interactions with extra-vestibular mechanisms.

Finally, it is worth mentioning that the promising results presented here were obtained with a first-of-its-kind, rudimentary vestibular implant, and during acute testing sessions. Indeed, it could be expected that both improved devices and sufficient training (i.e., when patients have enough time to adapt and use the full potential of the artificial vestibular information), would result in improved performance and rehabilitation prospects. We are therefore convinced that the vestibular implant is an evolutionary device with an immense clinical and research potential. Further research and development in this field are thus justified and warranted. 


\section{Acknowledgment}

The authors thank the technology transfer office of the University of Geneva (UNITEC) for their financial support for the development of the technical interface to couple the gyroscope to the cochlear implant processor through the INNOGAP proof- of-principle fund. 


\section{References}

1. Merfeld DM, Haburcakova C, Gong W, Lewis RF. Chronic vestibulo-ocular reflexes evoked by a vestibular prosthesis. IEEE Trans Biomed Eng 2007;54(6 Pt 1):1005-15.

2. Dai C, Fridman GY, Davidovics NS, Chiang B, Ahn JH, Della Santina CC. Restoration of 3D vestibular sensation in rhesus monkeys using a multichannel vestibular prosthesis. Hear Res 2011;281:74-83.

3. Perez Fornos A, Guinand N, Van de Berg R, Stokroos R, Micera S, Kingma H, et al. Artificial balance: restoration of the vestibulo-ocular reflex in humans with a prototype vestibular neuroprosthesis. Front Neurol 2014;5:66.

4. Phillips C, Defrancisci C, Ling L, Nie K, Nowack A, Phillips JO, et al. Postural responses to electrical stimulation of the vestibular end organs in human subjects. Exp Brain Res 2013;229: 181-95.

5. Guinand N, Van de Berg R, Cavuscens S, Stokroos RJ, Ranieri M, Pelizzone M, et al. Vestibular implants: 8 years of experience with electrical stimulation of the vestibular nerve in 11 patients with bilateral vestibular loss. ORL J Otorhinolaryngol Relat Spec 2015;77:227-40.

6. Guinand N, Boselie F, Guyot JP, Kingma H. Quality of life of patients with bilateral vestibulopathy. Ann Otol Rhinol Laryngol 2012;121:471-7.

7. Sun DQ, Ward BK, Semenov YR, Carey JP, Della Santina CC. Bilateral vestibular deficiency: quality of life and economic implications. JAMA Otolaryngol. Head Neck Surg 2014;140:527-34.

8. Grossman GE, Leigh RJ, Bruce EN, Huebner WP, Lanska DJ. Performance of the human vestibuloocular reflex during locomotion. J Neurophysiol 1989;62:264-72.

9. Berg van de R, Guinand N, Nguyen K, Ranieri M, Cavuscens S, Guyot JP, et al. The vestibular implant: frequency-dependency of the electrically evoked vestibulo-ocular reflex in humans. Front Syst Neurosci 2015;8:255.

10. Gong W, Merfeld DM. System design and performance of a unilateral horizontal semicircular canal prosthesis. IEEE Trans Biomed Eng 2002;49:175-81.

11. Guinand N, Pijnenburg M, Janssen M, Kingma H. Visual acuity while walking and oscillopsia severity in healthy subjects and patients with unilateral and bilateral vestibular function loss. Arch Otolaryngol Head Neck Surg 2012;138:301-6.

12. Schubert MC, Migliaccio AA, Della Santina CC. Dynamic visual acuity during passive head thrusts in canal planes. J Assoc Res Otolaryngol 2006;7:329-38.

13. Vital D, Hegemann SC, Straumann D, Bergamin O, Bockisch CJ, Angehrn D, et al. A new dynamic visual acuity test to assess peripheral vestibular function. Arch Otolaryngol Head Neck Surg 2010;136: 686-91.

14. Lewis RF, Haburcakova C, Gong W, Makary C, Merfeld DM. Vestibuloocular reflex adaptation investigated with chronic motion-modulated electrical stimulation of semicircular canal afferents. J Neurophysiol 2010;103:1066-79.

15. Lambert S, Sigrist A, Delaspre O, Pelizzone M, Guyot JP. Measurement of dynamic visual acuity in patients with vestibular areflexia. Acta Otolaryngol 2010;130:820-3.

16. Hillman EJ, Bloomberg JJ, McDonald PV, Cohen HS. Dynamic visual acuity while walking in normals and labyrinthine-deficient patients. J Vestib Res 1999;9:49-57.

17. Kos MI, Feigl G, Anderhuber F, Wall C, Fasel JH, Guyot JP. Transcanal approach to the singular nerve. Otol Neurotol 2006;27:542-6.

18. Berg van de R, Guinand N, Guyot JP, Kingma H, Stokroos RJ. The modified ampullar approach for vestibular implant surgery: feasibility and its first application in a human with a long-term vestibular loss. Front Neurol 2012;3:18.

19. Pelizzone M, Perez Fornos A, Ranieri M, Cavuscens S. Device and Method for Electrical Stimulation of Neural or Muscular Tissue EP12153300.x- 1652, Geneva. 2013.

20. Suzuki JI, Cohen B, Bender MB. Compensatory eye movements induced by vertical semicircular canal stimulation. Exp Neurol 1964;9:137-60.

21. Guyot JP, Sigrist A, Pelizzone M, Kos MI. Adaptation to steady- state electrical stimulation of the vestibular system in humans. Ann Otol Rhinol Laryngol 2011;120:143-9. 
22. Brainard DH. The psychophysics toolbox. Spat Vis 1997;10:433-6.

23. Pelli DG. The VideoToolbox software for visual psychophysics: transforming numbers into movies. Spat Vis 1997;10:437-42.

24. Kleiner M, Brainard DH, Pelli DG. What's new in Psychtoolbox-3? Perception 2007;36:1-65. ECVP Abstract Supplement.

25. Holladay JT. Proper method for calculating average visual acuity. J Refract Surg 1997;13:388-91.

26. Cushing SL, Pothier D, Hughes C, Hubbard BJ, Gordon KA, Papsin BC. Providing auditory cues to improve stability in children who are deaf. Laryngoscope 2012;122, S101-2.

27. Parkes WJ, Gnanasegaram JJ, Cushing SL, McKnight CL, Papsin BC, Gordon KA. Vestibular evoked myogenic potential testing as an objective measure of vestibular stimulation with cochlear implants. Laryngoscope 2017;127(2):E75-81. 



\section{Chapter 6}

Final discussion and valorisation 



\section{Introduction}

Inspired from the success of cochlear implants over the last decades in hearing rehabilitation for deaf patients, the concept of the vestibular implant had emerged as a potentially extremely interesting therapeutic option for the rehabilitation of the vestibular function. Persuasive results obtained in animal research led to the transfer to human research, not without having spent considerable amount of time convincing the local ethical committee. Respecting very strict inclusion criteria limiting the research protocol candidacy to patients suffering from a severe bilateral vestibular loss and a concomitant deafness, the first experiments using electrical stimulation of the vestibular nerve were performed at the ENT Department of the University Hospitals of Geneva in 2004 (personal communication).

At the beginning of my thesis, in 2009, I was aware of those extraordinary results. Imagine, it was possible to generate controlled eye movements by using electrical stimulation of the vestibular nerve in patients with a bilateral vestibular loss! Naturally, many questions emerged. They were the driving force behind this thesis in which a selection of essential issues of the vestibular implant project are addressed in a logical, chronological order.

\section{Is there actually a need for such a device?}

This seems to be the logical question before starting the development of any medical device. When asked, many clinicians were not aware of the existence of a condition such as the bilateral vestibular loss. What could they then say about the patient's complaints? Patients with balance disorders are commonly taken care of by ear nose and throat specialists or neurologists. But even among this subset of physicians the clinical relevance of a bilateral vestibular loss was not uniformly recognized. Several options are presented to us. First, the bilateral vestibular loss is indeed not relevant for Humans and thanks to the redundancy of the multidimensional balance system there is no relevant impact on its function. Second, as reported by some authors, current therapeutic concepts are efficient to tackle the handicap caused by the bilateral vestibular loss. Third, no one is really interested by this group of patients, possibly because there is no efficient treatment available. Fourth, patients with a bilateral vestibular loss have a significant handicap with an impact on their daily life without available efficient treatments. Based on our clinical experience we have always been convinced of the last option. For us the answer to the initial question was a clear yes. Yet we had to demonstrate this. As from the beginning our target group for the vestibular implant project has been the group of patient with a bilateral vestibular loss we decided to assess their quality of life. Chapter 2 deals with this issue. Results demonstrate the significant impact of the bilateral loss of the vestibular function on the quality of life. This has been corroborated in later studies which also 
revealed the significant social and economical burden on affected patients and on society. ${ }^{1}$ Furthermore, in our study a validated handicap index indicated that most of those patients have a moderate to severe handicap. This is worse compared to reported handicap index for other common inner ear pathologies such as superior canal dehiscence or unilateral vestibular loss for example. ${ }^{2,3}$ For those both conditions there are available treatments. In conclusion, yes there is a need for therapeutic options for the group of patients presenting a bilateral vestibular loss. Behind the initial question a more global issue appears: the recognition of vestibular disorders and in particular of the bilateral vestibular loss. Fortunately, promising progress is being made. Indeed in 2017, for the first time a consensus document of the Classification Committee of the Bárány Society dealing with the diagnostic criteria of the bilateral vestibulopathy was released (http://www.jvr-web.org/images/ Bilateral_vestibulopathy\%202017.pdf). This will be a very important reference to standardize research on this topic. In the effort towards a better recognition of vestibular disorders, emphasis should be placed on pre and post graduate education for health specialists, and on dissemination via publications, conferences, media appearances in order to reach as large audience as possible. At a world level and in the same way than for other sensory deficits like deafness and blindness, the bilateral vestibular loss (you notice that there is no specific word to describe this condition...) should be listed WHO health topics (http://www.who.int/topics/deafness/en/ + http://www.who.int/topics/blindness/en/). Together with the vestibular implant project those actions could endeavor to change patient's life.

\section{How to assess the benefit of the vestibular implant?}

In order to assess the effect of a treatment it is ideal to have valid objective measurements. This is not trivial with the vestibular system whose evaluation is complex. First the adequate stimuli for the system are accelerations. Unfortunately, it is virtually impossible to deliver such motion stimuli selectively to the vestibular system without activating other sensory systems. Second, there are various outcome parameters which give indications on different aspects of the vestibular function. Recording of eye movements induced by the vestibulo-ocular reflex inform us about gaze stabilization abilities, recording of body sway gives indication about postural control abilities, recording of motion perception threshold involves cortical integration of vestibular information, and other tests such as the measurement of vestibular evoked myogenic potentials gives indication on the integrity of the vestibulo- spinal pathways and presumably on the abilities for head and body stabilization. Third, no significant correlation between symptoms and most of those outcomes is found in patients with vestibular disorders. Therefore we focused our interest on a test assessing gaze stabilization during walking. Although it is not a purely vestibular test we hypothesized that this test would be very sensitive to depict 
a bilateral vestibular loss. Available data in the literature indicated that it was the case. ${ }^{4}$ Visual acuity was measured in standardized walking conditions on a treadmill at different velocities and compared to static values. This study composes the second Chapter of this thesis. Results showed that our dynamic visual acuity test was very sensitive. Significant drop of the visual acuity where found at walking velocity as low as $2 \mathrm{~km} / \mathrm{h}$. This indicates that this test should be suited to evaluate the benefit of a vestibular implant whose one of the aims is to restore efficient gaze stabilization. The results also showed that there were no significant differences between patients with a unilateral vestibular loss and healthy subjects, even at quite high walking velocities. This indicates that unilateral restoration of the vestibular function with a vestibular implant could be sufficient to reach a significant improvement of gaze stabilization. This supports our concept of a unilateral vestibular implant. On the other hand no correlation was found between the amount of visual acuity loss and the symptom intensity. Tolerance to retinal slip (image instability in dynamic conditions) may vary from subject to subject. Once more it highlights the difficulty to assess the balance system. With this test we now have an interesting tool to help selecting adequate candidates for a vestibular implantation and to assess the implant performance.

\section{What is the potential of each implanted electrode?}

Promising results obtained in acute experiments have paved the way for the permanent implantation of the first vestibular implant prototypes, which were modified cochlear implant stimulators with one electrode made available for vestibular stimulation by removing it from the main cochlear electrode array. Using custom modified devices was a crucial strategic choice. This allowed us to avoid the time-consuming, laborious and costly procedures associated with the development of a medical device such as a vestibular implant from scratch. This was possible thanks to a progressive, intelligent, trustworthy collaboration with a private company. This should be noted as it constitutes a fundamental aspect of the project success. In this framework, new versions of the prototype of the vestibular implant were developed. This evolutionary process is still under way, both in the design of the electrodes and the external processor, and should make possible to obtain a commercial version in the near future. Up to know, 13 patients have been implanted with different versions of the vestibular implant prototype. There were no surgical complications and all of them could benefit from the cochlear implant. This last point was a strong argument to obtain the approval of the ethical committee for the permanent implantation of the vestibular implant prototypes. In fact, at the time of writing Chapter 4, 11 patients had been implanted. The purpose of this chapter is to report selective significant objective parameters obtained for each electrode. Interpretation of those parameters should give indications on the potential of each electrode towards rehabilitation of the vestibular function. Three different version of vestibular implant prototypes were 
implanted in this group of 11 patients, for a total of 24 vestibular electrodes. Although all implantations in our project were made in deaf ears, hearing preservation is a crucial issue for the long term success of the vestibular implant, as the majority of patients with a bilateral vestibular loss has a normal hearing or moderate hearing loss. Depending on the post implantation level of hearing preservation, a purely vestibular implant without cochlear electrode could also be foreseen. If the therapeutic benefit obtained with a vestibular implant is sufficient, patients with bilateral vestibular loss might be ready to take the risk to lose their hearing on the implanted side. Nevertheless, extralabyrinthine surgical approaches, thought to be less traumatic than intralabyrinthine approaches, were initially developed and used for the first experiments. ${ }^{5,6}$ In light of animal research findings showing that at least in certain cases it was possible to preserve the hearing after implantation of intralabyrinthine electrodes, ${ }^{7}$ specific surgical routes for such an implantation were described and adequate vestibular implant prototype were developed. ${ }^{8}$ Among the 11 patients, 4 have vestibular implant prototypes with 1 extralabyrinthine electrode, 1 with 2 extralabyrinthine electrodes and 6 with 3 intralabyrinthine electrodes. Results show that electrical stimulation is a safe and effective means to activate the vestibular system even in a heterogeneous patient population with very different etiologies and disease durations. However, for each electrode, the electrical dynamic ranges, as well as the amplitude of the obtained eye movements were variable. The distance between the electrode and the neural target is of crucial importance to achieve a selective and efficient electrical stimulation. Towards this goal several aspects should be considered: 1 . The optimization of the positioning of the electrodes by refining their design and by monitoring intraoperatively the activity of the vestibular nerve during electrode insertion, 2. The increase of anatomic knowledge by creating accurate 3D models based on high tech imaging, 3. The establishment of standard surgical approaches, 4 . The exclusion of patients with etiologies inducing neural degeneration. Those issues appear to be essential to turn the vestibular implant prototype into a successful artificial balance organ.

\section{Can we make something functionally useful out of it?}

Artificial restoration of vestibular reflexes is a remarkable achievement. Nevertheless it does not guarantee a useful functional restitution for the patient. The aim of this final part of the thesis was to demonstrate a useful benefit for implanted patients by using a selected subset of implanted electrodes and the test described in Chapter 3. Can the dynamic visual acuity of implanted patients be improved? For this purpose we chose to use an out the shelf motion sensor which was fixated to the patients head. Motion information captured by this sensor was transmitted to a regular cochlear implant processor, which in turns controlled the electrical stimulation delivered by the implanted stimulator to the ampullary branches of the vestibular nerve. In fact the 
strategy for transmitting the motion information to the processor has been patented by members of our research team (M. Pelizzone, A. Perez-Fornos, M. Ranieri and S. Cavuscens, Device for electrical stimulation of neural and/or muscular tissue, has signal processing unit to transform input signal received from signal sensors into modulated electrical output signal to be treated by speech processor., in, vol WO2014118094-A1; EP2762196-A1, 2013.). The main perturbations of the head during walking being vertical translation and head pitch, a subset of electrodes eliciting mainly vertical eye movements was selected for this experimental protocol. The hypothesis made was that those eye movements would compensate for the head movements allowing preservation of the visual acuity while walking. In all 6 implanted patients available for the study the dynamic visual acuity could be significantly improved or even normalized. This represents the first demonstration of a useful functional rehabilitation using a vestibular implant prototype. Results were obtained with a single active electrode by using one-dimensional motion information. It is remarkable that this somehow rudimentary motion information is sufficient in making a significant functional difference in gaze stabilization. We believe that brain plasticity plays a key role, which might mean that perfect restitution of the missing motion information to the central nervous system will not be necessary improve the balance function in a clinically relevant manner. In some respects this is the case for cochlear implants. Indeed a minimum of 6 or 8 independent cochlear electrodes are sufficient for an efficient hearing rehabilitation allowing the development of close to normal speech in a deaf child for instance. In conclusion, the answer is yes, motion modulated electrical stimulation of the ampullary branches of the vestibular nerve can restore a functionally useful vestibular function. With this in mind, it is not anymore utopic to foresee the possibility of having a vestibular implant on the market within the following years.

We hope the horizon of patients with a bilateral vestibular loss will be more stable...

\section{Remarks}

On purpose this thesis has been focused on chosen aspects of the vestibular implant project which should provide the reader an interesting, significant glimpse into it. An aspect of interest left somewhat aside is that of more fundamental research. Allowing independent selective activation of the vestibular system, which is unprecedented, the vestibular implant has opened doors in the quest for understanding the physiology of vestibular system.

More information about the project can be found in the numerous publications from our research group. 


\section{References}

1. Sun DQ, Ward BK, Semenov YR, Carey JP, Della Santina CC. Bilateral Vestibular Deficiency: Quality of Life and Economic Implications. JAMA Otolaryngol Head Neck Surg 2014;140:527-34.

2. Crane BT, Minor LB, Carey JP. Superior canal dehiscence plugging reduces dizziness handicap. Laryngoscope 2008;118:1809-13.

3. Jacobson GP, Calder JH. Self-perceived balance disability/handicap in the presence of bilateral peripheral vestibular system impairment. J Am Acad Audiol 2000;11:76-83.

4. Lambert S, Sigrist A, Delaspre O, Pelizzone M, Guyot JP. Measurement of dynamic visual acuity in patients with vestibular areflexia. Acta Otolaryngol 2010;130:820-3.

5. Kos MI, Feigl G, Anderhuber F, Wall C, Fasel JH, Guyot JP. Transcanal approach to the singular nerve. Otol Neurotol 2006;27:542-6.

6. Feigl GC, Fasel JH, Anderhuber F, Ulz H, Rienmuller R, Guyot JP, et al. Superior vestibular neurectomy: a novel transmeatal approach for a denervation of the superior and lateral semicircular canals. Otol Neurotol 2009;30:586-91.

7. Dai C, Fridman GY, Della Santina CC. Effects of vestibular prosthesis electrode implantation and stimulation on hearing in rhesus monkeys. Hear Res 2011;277:204-10.

8. van de Berg R, Guinand N, Guyot JP, Kingma H, Stokroos RJ. The modified ampullar approach for vestibular implant surgery: feasibility and its first application in a human with a long-term vestibular loss. Front Neurol 2012;3:18. 
Summary 



\section{Summary}

The vestibular system, located in the inner ear acts like a motion sensor. It provides the central nervous system with information about head movements and position. Together with vision and proprioception, it is an essential part of the multidimensional balance system. It allows for the generation of vestibular reflexes, which are among the fastest in the human body and results in efficient gaze stabilization and postural control. Vestibular information is influencing many other functions, such as motion perception, spatial orientation, blood regulation, memory, sleep, bone metabolism and many others. As the vestibular system functions automatically and unconsciously, as long as it is normally functioning we are not aware of it. It is perhaps one of the reasons why the vestibular system is not well recognized, not only in the general population but also among health specialists. It follows that problems associated with the loss of its function, in particular in case of bilateral involvement, are not optimally taken care of. In this thesis we demonstrated that patients with a bilateral loss of the vestibular function present a moderate to severe handicap leading to a significant decrease of their quality of life. In about $50 \%$ of the cases the etiology remains unknown. Unfortunately there is currently no evidence of efficient treatment. Based on the success of cochlear implants for hearing rehabilitation in deaf patients, the concept of a vestibular implant to restore the vestibular function has emerged. The hypothesis is that using motion modulated electrical stimulation of the vestibular nerve, the vestibular implant could provide the central nervous system with sufficient motion information to restore a useful vestibular function. The vestibular implant prototype consists of a modified cochlear implant with one up to three electrodes removed from main cochlear array and put in contact with terminal ampullary branches of the vestibular nerve. Different surgical approaches were developed for this purpose. Finally a motion sensor is fixated to the head and feeds a regular cochlear implant processor with motion information. Currently 13 patients have been implanted. With the majority of the available electrodes it is possible to elicit controlled eye movements which correspond to the restoration of the vestibulo-ocular reflex. There is a high intra individual and inter individual variability in obtained responses. The best electrodes generate responses in line with our knowledge of the vestibular physiology, indicating that a useful functional rehabilitation could be possible. To demonstrate this, a test assessing the visual acuity while walking was developed. Indeed, one of the main complaints of patients suffering a bilateral vestibular loss is blurred vision when walking. In this test patient's visual acuity was measured at different walking velocities and compared to values obtained while standing still. Compared to healthy subjects, patients with a bilateral vestibular loss had a pathological drop of their visual acuity at speed as slow as $2 \mathrm{~km} / \mathrm{h}$. Patients with a unilateral vestibular loss were also tested. The fact that their visual acuity was preserved even at high walking speed indicates that a unilateral 
restitution of the vestibular function could be sufficient to improve significantly gaze stabilization. In the last part of the thesis we actually tested this assumption. 6 implanted patients performed the same dynamic visual acuity test. In all of them, a significant improvement or even a normalization of the visual acuity while walking was found. This demonstrated for the first time a clinically useful rehabilitation of the vestibular function. Based on those promising results and the gained experience, the vestibular implant prototype design will be refined, the processor software upgraded, the electrode positioning optimized and the patients' selection will be targeted. Hopefully the device will be available on the market in the coming years. In parallel, the effort to improve the awareness of vestibular disorders will be pursued and the vestibular implant prototype will give us unique opportunity to explore the vestibular physiology beyond the traditional boundaries. 
List of publications 



\section{List of publications}

\section{Articles in peer reviewed journals}

1. Cao Van H, Guinand N, Damis E, Mansbach AL, Poncet A, Hummel T, Landis BN.Olfactory stimulation may promote oral feeding in immature newborn: a randomized controlled trial. Eur Arch Otorhinolaryngol. 2018;275(1):125-9. (IF: n.a.)

2. van de Berg R, Guinand N, Ranieri M, Cavuscens S, Khoa Nguyen TA, Guyot JP, Lucieer F, Starkov D, Kingma $H$, van Hoof $M$, Perez-Fornos A. The Vestibular Implant Input Interacts with Residual Natural Function. Front Neurol. 2017;8:644. (IF : 3.50)

3. Lucieer F, Duijn S, Van Rompaey V, Pérez Fornos A, Guinand N, Guyot JP, Kingma $H$, van de Berg R. Full Spectrum of Reported Symptoms of Bilateral Vestibulopathy Needs Further Investigation-A Systematic Review. Front Neurol. 2018;9:352. (IF : 3.50)

4. van den Boogert T, van Hoof M, Handschuh S, Glueckert R, Guinand N, Guyot JP, Kingma H, Perez-Fornos A, Seppen B, Johnson Chacko L, Schrott-Fischer A, van de Berg R. Optimization of 3D-Visualization of Micro-Anatomical Structures of the Human Inner Ear in Osmium Tetroxide Contrast Enhanced Micro-CT Scans. Front Neuroanat. 2018;12:41. (IF : 3.15)

5. Guinand N, Van de Berg R, Cavuscens S, Ranieri M, Schneider E, Lucieer F, Kingma H, Guyot JP, Pérez Fornos A. The Video Head Impulse Test to Assess theEfficacy of Vestibular Implants in Humans. Front Neurol. 2017;8:600. (IF : 3.55)

6. van de Berg R, Guinand N, Ranieri M, Cavuscens S, Khoa Nguyen TA, Guyot JP, Lucieer F, Starkov D, Kingma $H$, van Hoof $M$, Perez-Fornos A. The Vestibular Implant Input Interacts with Residual Natural Function. Front Neurol. 2017;8:644 (IF:3.55)

7. Nguyen TAK, Cavuscens S, Ranieri M, Schwarz K, Guinand N, van de Berg R, van den Boogert T, Lucieer F, van Hoof M, Guyot JP, Kingma H, Micera S, Perez Fornos A. Characterization of Cochlear, Vestibular and Cochlear-Vestibular Electrically Evoked Compound Action Potentials in Patients with a VestibuloCochlear Implant. Front Neurosci. 2017;11:645. (IF : 3.57) 
8. Perez-Fornos A, van de Berg R, Ranieri M, Cavuscens S, Stokroos R, Kingma H, Guyot JP, Guinand N. The vestibular implant: a probe in orbit around the human balance system. J Vestib Res. 2017;27(1):51-61. (IF: 0.9)

9. Dulguerov N, Guinand N, Courvoisier D, Landis BN, Lacroix JS, Hauser C. Rhinophototherapy in chronic rhinosinusitis: a double blind randomized placebocontrolled trial. Rhinology. 2017;55(2):106-12. (IF: 2.35)

10. van de Berg R, Lucieer F, Guinand N, van Tongeren J, Gerorge E, Guyot JP, Kingma $H$, van Hoof $M$, Temel $Y$, van Overbeeke J, Perez Fornos A, Stokroos R. The vestibular implant: Hearing preservation during intralabyrinthine electrode insertion - A case report. Front. Neurol. 2017;8:137 (IF: 3.55)

11. Morrison M, Guinand N, Alianou A, Becker M, SennP. Spontaneous intracochlear hemorrhage leading to sensorineural hearing loss. Annals of Otolaryngology and Rhinology, 2017 Jan. (IF: 1.38)

12. Guinand N, Ranieri M, Cavuscens $S$, van den Berg R, Kingma H, Pelizzone $M$, Guyot JP, Pérez Fornos A. Restoring dynamic visual acuity with a vestibular implant. Front Neurosci 2016;10:577 (IF: 3.57)

13. Nguyen TAK, DiGiovanna J, Cavuscens S, Ranieri M, van de Berg R, Guinand $\mathbf{N}$, Carpaneto J, Kingma H, Guyot JP, Micera S, and Perez-Fornos A. Effectiveness of Pulse Amplitude Modulation and Pulse Rate Modulation for a Human Vestibular Implant during Acute Electrical Stimulation. J Neural Eng. 2016;13(4):046023. (IF: 3.47)

14. DiGiovanna J, Nguyen TA, Guinand N, Pérez-Fornos A, Micera S. Neural Network Model of Vestibular Nuclei Reaction to Onset of Vestibular Prosthetic Stimulation. Front Bioeng Biotechnol. 2016;4:34. (IF: n.a.)

15. Lucieer F, Vonk P, Guinand N, Stokroos R, Kingma H, van de Berg R. Bilateral Vestibular Hypofunction: Insights in Etiologies, Clinical Subtypes, and Diagnostics. Front Neurol. 2016;7:26. (IF: 3.55)

16. Pittet MP, Idan RB, Kern I, Guinand N, Van HC, Toso S, Fluss J. Acute cortical deafness in a child with MELAS syndrome. J Inherit Metab Dis. 2016;39(3):465-6. (IF: 3.97) 
17. Guyot JP, Perez Fornos A, Guinand N, van de Berg R, Stokroos R, Kingma H. Vestibular assistance systems: promises and challenges. J Neurol. 2016;263 Suppl 1:30-5. (IF: 3.39)

18. Guinand N, van de Berg R, Cavuscens S, Stokroos R, J, Ranieri M, Pelizzone M, Kingma H, Guyot J, P, Perez-Fornos A, Vestibular Implants: 8 Years of Experience with Electrical Stimulation of the Vestibular Nerve in 11 Patients with Bilateral Vestibular Loss. ORL 2015;77:227-40 (IF: 1.06)

19. Gay A, Guinand N, Miffon M, Guyot J, -P, Perception of Discomfort Caused by a Unilateral Hearing Loss in People Suffering from a Total Bilateral Vestibular Loss. ORL 2015;77:248-53 (IF: 1.06)

20. Guinand N, van de Berg R, Ranieri M, Cavuscens S, DiGiovanna J, Nguyen TA, Micera S, Stokroos R, Kingma H, Guyot JP, Perez Fornos A. Vestibular implants: Hope for improving the quality of life of patients with bilateral vestibular loss. Conf Proc IEEE Eng Med Biol Soc. 2015;2015:7192-5. (IF: n.a.)

21. van de Berg R, Guinand N, Nguyen TA, Ranieri M, Cavuscens S, Guyot JP,Stokroos $\mathrm{R}$, Kingma $\mathrm{H}$, Perez-Fornos $\mathrm{A}$. The vestibular implant: frequency-dependency of the electrically evoked vestibulo-ocular reflex in humans. Front Syst Neurosci. 2015;8:255 (IF: n.a.)

22. Perez Fornos A, Guinand N, van de Berg R, Stokroos R, Micera S, Kingma H, Pelizzone M, Guyot JP. Artificial balance: restoration of the vestibulo-ocular reflex in humans with a prototype vestibular neuroprosthesis. Front Neurol. 2014;5:66. (IF: n.a.)

23. Pelizzone M, Fornos AP, Guinand N, van de Berg R, Kos I, Stokroos R, Kingma H, Guyot JP. First functional rehabilitation via vestibular implants. Cochlear Implants Int. 2014;15 Suppl 1:S62-4 (IF: n.a.)

24. Guinand N, Boselie F, Guyot JP, Kingma H. Quality of life of patients with bilateral vestibulopathy. Ann Otol Rhinol Laryngol. 2012;121(7):471-7. (IF: 1.21)

25. Heiser C, Landis BN, Giger R, Cao Van H, Guinand N, Hörmann K, Stuck BA. Taste disorders after tonsillectomy: a long-term follow-up. Laryngoscope. 2012;122(6): 1265-6. (IF: 1.98) 
26. Guinand N, Pijnenburg M, Janssen M, Kingma H. Visual acuity while walking and oscillopsia severity in healthy subjects and patients with unilateral and bilateral vestibular function loss. Arch Otolaryngol Head Neck Surg. 2012;138(3):301-6. (IF: 1.78)

27. van de Berg R, Guinand N, Guyot JP, Kingma H, Stokroos RJ. The modified ampullar approach for vestibular implant surgery: feasibility and its first application in a human with a long-term vestibular loss. Front Neurol. 2012;3:18. (IF: n.a.)

28. Guinand N, Guyot JP, Kingma H, Kos I, Pelizzone M. Vestibular implants: the first steps in humans. Conf Proc IEEE Eng Med Biol Soc. 2011;2011:2262-4. (IF: n.a.)

29. Hoffmann KP, Poppendieck W, Tätzner S, DiGiovanna J, Kos MI, Guinand N, Guyot JP, Micera S. 3D hybrid electrode structure as implantable interface for a vestibular neural prosthesis in humans. Conf Proc IEEE Eng Med Biol Soc. 2011;2011:1073-6. (IF: n.a.)

30. van de Berg R, Guinand N, Stokroos RJ, Guyot JP, Kingma H. The vestibular implant: quo vadis? Front Neurol. 2011;2:47. (IF: n.a.)

31. Heiser C, Landis BN, Giger R, Cao Van H, Guinand N, Hörmann K. Taste Disturbance Following Tonsillectomy-A Prospective study. Laryngoscope 2010;120(10):2119-24 (IF: 2.10)

32. Guinand N, Just T, Stow NW, Cao Van H, Landis BN. Cutting the chorda tympani is more than a matter of taste. J Laryngol Otol. 2010:1-4 (IF: 0.7)

33. Landis BN, Guinand N. Selective taste disorder after temporal bone fracture. J Clin Neurosci 2009;16(4):605. (IF: 1.17)

34. Landis BN, Cao Van H, Guinand N, Horvath J, Savva E, Hugentobler M, Lacroix JS, Burkhard P. Retronasal olfactory function in Parkinson's disease. Laryngoscope. 2009;119(11):2280-3 (IF: 2.02)

Manuscripts submitted in and in preparation (peer reviewed journals)

1. Perez-Fornos A, Ranieri M, Cavuscens S, van den Berg R, Kingma H, Pelizzone M, Guyot JP, Guinand N. Artificial restoration of the vestibulo-collic reflex with a vestibular implant.(in preparation) 


\section{Articles in other journals}

1. Todic J, Guyot JP, Perez-Fornos A, Cavuscens S, Ranieri M, Merfeld D, Lewis R, Guinand N. Vestibular and Visual Stimulation: Simultaneous perception or not? Rev Med Suisse. 2016;12(533):1650-2.

2. Guyot JP, Guinand N. [Vestibular function: the 6th sense... ignored]. Rev Med Suisse. 2015;11(488):1782, 1784-6.

3. Pelizzone M, Fornos AP, Guinand N, van de Berg R, Kos I, Stokroos R, Kingma H, Guyot JP. First functional rehabilitation via vestibular implants. Cochlear Implants Int. 2014;15 Suppl 1:S62-4.

4. Vankatova L, Cao Van H, Perez Fornos A, Guinand N. [Cochlear implantation better safe than sorry]. Rev Med Suisse. 2014;10(444):1820,1822-3.

5. Guinand N, Dulguerov P, Giger R. Radiation-induced xerostomia: prevention, treatment, perspectives. Rev Med Suisse. 2007;3(127):2225-9.

6. Guinand N, Guyot JPh, Kos MI. Les paragangliomes jugulaires et tympaniques. Rev Med Suisse. 2008;4:2094-7.

7. Guinand N, Guyot JPh. Vertiges d'origine cervicale, mythe ou réalité?. Rev Med Suisse. 2009;5:1922-4.

8. Guinand N, Truffert A, Lacroix JS, Landis BN. Fonction gustative dans le cadre de la paralysie faciale idiopathique. Schweizerische Medizinische Forum 2008;8; (Suppl 41):88s-90s.

9. Guinand N, Hainard-Jaquier S, Dulguerov P. Laryngoplastie par injection de graisse autologue. Schweizerische Medizinische Forum 2006;6:(Suppl 29): 10s-2s.

10. Guinand N, Valentini G, Mazaud Ch, Kos MI, Guyot JP, Pelizzone M. Performances auditives avant et après implantation cochléaire. Schweizerische Medizinische Forum 2010;10;(Suppl. 51), 1S-3S.

11. Guinand N, Mazaud C, Guyot JP, Kos I, Pelizzone M. Utilisation du téléphone chez les patients porteurs d'un implant cochléaire. Schweizerische Medizinische Forum 2009;9;(Suppl 49). 

Curriculum vitae 



\section{Curriculum vitae}

Nils Guinand was born on June $19^{\text {th }} 1977$ in Bern, Switzerland. He graduated from the Medical School of the University of Bern in 2002. His clinical training in Otorhinolaryngology and Head \& Neck Surgery was carried out at the University Hospitals of Geneva, the University Hospital of Canton Vaud in Lausanne and the Ziegler Hospital in Bern. Thanks to grants of a private Foundation and of the European Union (FET7) he has spent 2 years (2009-2011) as a clinical research fellow in the vestibular lab of Professor Herman Kingma in the Otorhinolaryngology and Head \& Neck Surgery Department at Maastricht University Medical Center in The Netherlands. During those 2 years he built the basis towards the current thesis. After returning in Geneva he received a full training in otology and otoneurology. Since January 2017 is the Head of the Otoneurolgy Unit of the Otorhinolaryngology and Head \& Neck Surgery Department at the University Hospitals of Geneva. He obtained the Swiss Diploma of ORL specialist in 2011. In parallel, he received the Swiss Diploma in Head and Neck Surgery in 2015. He obtained the Medical Doctorate of the University of Geneva in 2015 ("Bilateral vestibular loss: impact on the quality of life"). He received the Harold Schuhknecht Travel Award (Thirteenth Triennal Meeting of The International Otopathology Society, Boston, US) in 2013 and the Academy Award of the European Academy of Otology and Neurootology in 2014 for the work on the development of the vestibular implant. 

Acknowledgements 



\section{Acknowledgements}

Thank you to all members of the Geneva-Maastricht team! The networking of ideas, intelligence, dedication, work capacity, innovation, enthusiasm, perseverance has made this fascinating vestibular implant project a success!

Thanks in particular to Jean-Philippe Guyot who has given me the opportunity to be part of the ENT team in his Service, an ideal environement for learning, with a noble vision of the practice of medicine. And latter, for having involved me in the vestibular implant project and for having helped me to find the ressources to make my 2 years stay in the Netherlands confortable for my family and myself. This is unvaluable.

Herman Kingma, thanks for welcoming me warmly and for openning widely the doors of your lab. I'm very gratefull for the time spent in Maastricht. Your knowledge and your positive intelligence have impacted my spirit forever.

Angelica, thanks... :)

Raymond, congratulations and thanks for all your work!

Maurizio, Samuel thanks for your dedication to the project and your multifaceted input.

Marco Pellizone, thank you for sharing your intelligence.

Izabel Kos, thank you for leting me navigate in the temporal bone.

Christel, Bram, Mark and Frans thank you for helping me to collect data.

Maurice, thanks for all the fruitful talks.

Robert, thanks for all your higly valuable advices.

Marie-Cécile, Elen, Olga thanks for your expertise, enthusiasm and flexibility!

Rick, Dan, thanks for sharing and discussing ideas and concepts on the topic.

Jack, Khoa, thanks for the many years of collaboration.

Silvestro, thanks for leading CLONS. 
Medel, vestibular team, thanks for the now long time fruitful relationship.

Grégory, René thanks for the skilled technical assistance.

Bart, thanks for your input.

Alain, thanks for helping to launch the first experiments.

Didier, Pierre André, thanks for your generosity.

Hélène, Evan, Noémie thanks for your support.

Lina and Pierre, my parents, thanks for your education and your presence. 
